\title{
The Standard Model of Electroweak Interactions
}

\author{
A. Pich \\ IFIC, University of València - CSIC, \\ València, Spain
}

\begin{abstract}
Gauge invariance is a powerful tool to determine the dynamical forces among the fundamental constituents of matter. The particle content, structure and symmetries of the Standard Model Lagrangian are discussed. Special emphasis is given to the many phenomenological tests which have established this theoretical framework as the Standard Theory of the electroweak interactions: electroweak precision tests, Higgs searches, quark mixing, neutrino oscillations. The present experimental status is summarized.
\end{abstract}

\section{Introduction}

The Standard Model (SM) is a gauge theory, based on the symmetry group $S U(3)_{C} \otimes S U(2)_{L} \otimes U(1)_{Y}$, which describes strong, weak and electromagnetic interactions, via the exchange of the corresponding spin-1 gauge fields: eight massless gluons and one massless photon, respectively, for the strong and electromagnetic interactions, and three massive bosons, $W^{ \pm}$and $Z$, for the weak interaction. The fermionic matter content is given by the known leptons and quarks, which are organized in a three-fold family structure:

$$
\left[\begin{array}{cc}
\nu_{e} & u \\
e^{-} & d^{\prime}
\end{array}\right], \quad\left[\begin{array}{cc}
\nu_{\mu} & c \\
\mu^{-} & s^{\prime}
\end{array}\right], \quad\left[\begin{array}{cc}
\nu_{\tau} & t \\
\tau^{-} & b^{\prime}
\end{array}\right],
$$

where (each quark appears in three different colours)

$$
\left[\begin{array}{cc}
\nu_{l} & q_{u} \\
l^{-} & q_{d}
\end{array}\right] \equiv\left(\begin{array}{c}
\nu_{l} \\
l^{-}
\end{array}\right)_{L}, \quad\left(\begin{array}{c}
q_{u} \\
q_{d}
\end{array}\right)_{L}, \quad l_{R}^{-}, \quad q_{u R}, \quad q_{d R}
$$

plus the corresponding antiparticles. Thus, the left-handed fields are $S U(2)_{L}$ doublets, while their righthanded partners transform as $S U(2)_{L}$ singlets. The three fermionic families in Eq. (1) appear to have identical properties (gauge interactions); they differ only by their mass and their flavour quantum number.

The gauge symmetry is broken by the vacuum, which triggers the Spontaneous Symmetry Breaking (SSB) of the electroweak group to the electromagnetic subgroup:

$$
S U(3)_{C} \otimes S U(2)_{L} \otimes U(1)_{Y} \quad \stackrel{\mathrm{SSB}}{\longrightarrow} S U(3)_{C} \otimes U(1)_{\mathrm{QED}} .
$$

The SSB mechanism generates the masses of the weak gauge bosons, and gives rise to the appearance of a physical scalar particle in the model, the so-called Higgs. The fermion masses and mixings are also generated through the SSB.

The SM constitutes one of the most successful achievements in modern physics. It provides a very elegant theoretical framework, which is able to describe the known experimental facts in particle physics with high precision. These lectures [1] provide an introduction to the SM, focussing mostly on its electroweak sector, i.e., the $S U(2)_{L} \otimes U(1)_{Y}$ part [2-5]. The strong $S U(3)_{C}$ piece is discussed in more detail in Refs. [6,7]. The power of the gauge principle is shown in Section 2, where the simpler Lagrangians of quantum electrodynamics and quantum chromodynamics are derived. The electroweak theoretical framework is presented in Sections 3 and 4, which discuss, respectively, the gauge structure and the SSB mechanism. Section 5 summarizes the present phenomenological status; it describes the main precision tests performed at the $Z$ peak and the tight constraints on the Higgs mass from direct 
searches. The flavour structure is discussed in Section 6, where knowledge of the quark mixing angles and neutrino oscillation parameters is briefly reviewed and the importance of $\mathcal{C P}$ violation tests is emphasized. Finally, a few comments on open questions, to be investigated at future facilities, are given in the summary. Some useful but more technical information has been collected in several appendices: a minimal amount of quantum field theory concepts are given in Appendix A; Appendix B summarizes the most important algebraic properties of $S U(N)$ matrices; and a short discussion on gauge anomalies is presented in Appendix C.

\section{Gauge Invariance}

\subsection{Quantum electrodynamics}

Let us consider the Lagrangian describing a free Dirac fermion:

$$
\mathcal{L}_{0}=i \bar{\psi}(x) \gamma^{\mu} \partial_{\mu} \psi(x)-m \bar{\psi}(x) \psi(x) .
$$

$\mathcal{L}_{0}$ is invariant under global $U(1)$ transformations

$$
\psi(x) \stackrel{U(1)}{\longrightarrow} \psi^{\prime}(x) \equiv \exp \{i Q \theta\} \psi(x),
$$

where $Q \theta$ is an arbitrary real constant. The phase of $\psi(x)$ is then a pure convention-dependent quantity without physical meaning. However, the free Lagrangian is no longer invariant if one allows the phase transformation to depend on the space-time coordinate, i.e., under local phase redefinitions $\theta=\theta(x)$, because

$$
\partial_{\mu} \psi(x) \stackrel{U(1)}{\longrightarrow} \exp \{i Q \theta\}\left(\partial_{\mu}+i Q \partial_{\mu} \theta\right) \psi(x) .
$$

Thus, once a given phase convention has been adopted at one reference point $x_{0}$, the same convention must be taken at all space-time points. This looks very unnatural.

The 'gauge principle' is the requirement that the $U(1)$ phase invariance should hold locally. This is only possible if one adds an extra piece to the Lagrangian, transforming in such a way as to cancel the $\partial_{\mu} \theta$ term in Eq. (6). The needed modification is completely fixed by the transformation (6): one introduces a new spin-1 (since $\partial_{\mu} \theta$ has a Lorentz index) field $A_{\mu}(x)$, transforming as

$$
A_{\mu}(x) \stackrel{U(1)}{\longrightarrow} \quad A_{\mu}^{\prime}(x) \equiv A_{\mu}(x)-\frac{1}{e} \partial_{\mu} \theta,
$$

and defines the covariant derivative

$$
D_{\mu} \psi(x) \equiv\left[\partial_{\mu}+i e Q A_{\mu}(x)\right] \psi(x),
$$

which has the required property of transforming like the field itself:

$$
D_{\mu} \psi(x) \stackrel{U(1)}{\longrightarrow}\left(D_{\mu} \psi\right)^{\prime}(x) \equiv \exp \{i Q \theta\} D_{\mu} \psi(x) .
$$

The Lagrangian

$$
\mathcal{L} \equiv i \bar{\psi}(x) \gamma^{\mu} D_{\mu} \psi(x)-m \bar{\psi}(x) \psi(x)=\mathcal{L}_{0}-e Q A_{\mu}(x) \bar{\psi}(x) \gamma^{\mu} \psi(x)
$$

is then invariant under local $U(1)$ transformations.

The gauge principle has generated an interaction between the Dirac fermion and the gauge field $A_{\mu}$, which is nothing else than the familiar vertex of Quantum Electrodynamics (QED). Note that the corresponding electromagnetic charge $Q$ is completely arbitrary. If one wants $A_{\mu}$ to be a true propagating field, one needs to add a gauge-invariant kinetic term

$$
\mathcal{L}_{\text {Kin }} \equiv-\frac{1}{4} F_{\mu \nu}(x) F^{\mu \nu}(x),
$$


where $F_{\mu \nu} \equiv \partial_{\mu} A_{\nu}-\partial_{\nu} A_{\mu}$ is the usual electromagnetic field strength which remains invariant under the transformation (7). A mass term for the gauge field, $\mathcal{L}_{m}=\frac{1}{2} m^{2} A^{\mu} A_{\mu}$, is forbidden because it would violate the local $U(1)$ gauge invariance; therefore, the photon field is predicted to be massless. Experimentally, we know that $m_{\gamma}<1 \cdot 10^{-18} \mathrm{eV}$ [8,9].

The total Lagrangian in Eqs. (10) and (11) gives rise to the well-known Maxwell equations:

$$
\partial_{\mu} F^{\mu \nu}=e J^{\nu} \equiv e Q \bar{\psi} \gamma^{\nu} \psi,
$$

where $J^{\nu}$ is the fermion electromagnetic current. From a simple gauge-symmetry requirement, we have deduced the right QED Lagrangian, which leads to a very successful quantum field theory.

\subsubsection{Lepton anomalous magnetic moments}

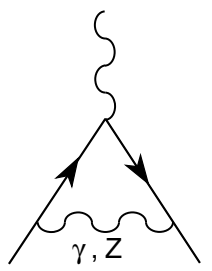

(a)

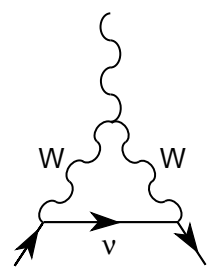

(b)

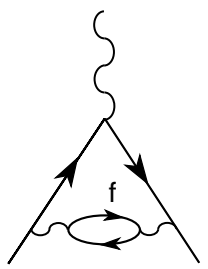

(c)

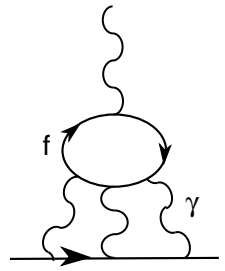

(d)

Fig. 1: Feynman diagrams contributing to the lepton anomalous magnetic moment.

The most stringent QED test comes from the high-precision measurements of the $e$ [10] and $\mu$ [11] anomalous magnetic moments $a_{l} \equiv\left(g_{l}^{\gamma}-2\right) / 2$, where $\vec{\mu}_{l} \equiv g_{l}^{\gamma}\left(e / 2 m_{l}\right) \vec{S}_{l}$ :

$$
a_{e}=(1159652180.73 \pm 0.28) \cdot 10^{-12}, \quad a_{\mu}=(11659208.9 \pm 6.3) \cdot 10^{-10} .
$$

To a measurable level, $a_{e}$ arises entirely from virtual electrons and photons; these contributions are fully known to $O\left(\alpha^{4}\right)$ and many $O\left(\alpha^{5}\right)$ corrections have been already computed [12-14]. The impressive agreement achieved between theory and experiment has promoted QED to the level of the best theory ever built to describe Nature. The theoretical error is dominated by the uncertainty in the input value of the QED coupling $\alpha \equiv e^{2} /(4 \pi)$. Turning things around, $a_{e}$ provides the most accurate determination of the fine structure constant [10, 15]:

$$
\alpha^{-1}=137.035999084 \pm 0.000000051 .
$$

The anomalous magnetic moment of the muon is sensitive to small corrections from virtual heavier states; compared to $a_{e}$, they scale with the mass ratio $m_{\mu}^{2} / m_{e}^{2}$. Electroweak effects from virtual $W^{ \pm}$and $Z$ bosons amount to a contribution of $(15.4 \pm 0.2) \cdot 10^{-10}$ [16-18], which is larger than the present experimental precision. Thus $a_{\mu}$ allows one to test the entire SM. The main theoretical uncertainty comes from strong interactions. Since quarks have electric charge, virtual quark-antiquark pairs induce hadronic vacuum polarization corrections to the photon propagator (Fig. 11 c). Owing to the nonperturbative character of the strong interaction at low energies, the light-quark contribution cannot be reliably calculated at present. This effect can be extracted from the measurement of the cross-section $\sigma\left(e^{+} e^{-} \rightarrow\right.$ hadrons $)$ and from the invariant-mass distribution of the final hadrons in $\tau$ decays, which unfortunately provide slightly different results [19]:

$$
a_{\mu}^{\text {th }}=\left\{\begin{array}{cc}
(11659180.2 \pm 4.9) \cdot 10^{-10} & \left(e^{+} e^{-} \text {data }\right) \\
(11659189.4 \pm 5.4) \cdot 10^{-10} & (\tau \quad \text { data })
\end{array}\right.
$$

The quoted uncertainties include also the smaller light-by-light scattering contributions (Fig. 1,d) [20]. The difference between the SM prediction and the experimental value (13) corresponds to $3.6 \sigma\left(e^{+} e^{-}\right)$ or $2.4 \sigma(\tau)$. New precise $e^{+} e^{-}$and $\tau$ data sets are needed to settle the true value of $a_{\mu}^{\text {th }}$. 


\subsection{Quantum chromodynamics}

\subsubsection{Quarks and colour}

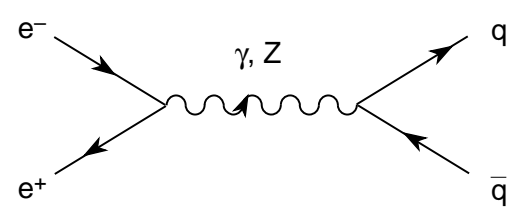

Fig. 2: Tree-level Feynman diagram for the $e^{+} e^{-}$annihilation into hadrons.

The large number of known mesonic and baryonic states clearly signals the existence of a deeper level of elementary constituents of matter: quarks. Assuming that mesons are $M \equiv q \bar{q}$ states, while baryons have three quark constituents, $B \equiv q q q$, one can nicely classify the entire hadronic spectrum. However, in order to satisfy the Fermi-Dirac statistics one needs to assume the existence of a new quantum number, colour, such that each species of quark may have $N_{C}=3$ different colours: $q^{\alpha}$, $\alpha=1,2,3$ (red, green, blue). Baryons and mesons are then described by the colour-singlet combinations

$$
B=\frac{1}{\sqrt{6}} \epsilon^{\alpha \beta \gamma}\left|q_{\alpha} q_{\beta} q_{\gamma}\right\rangle, \quad M=\frac{1}{\sqrt{3}} \delta^{\alpha \beta}\left|q_{\alpha} \bar{q}_{\beta}\right\rangle .
$$

In order to avoid the existence of non-observed extra states with non-zero colour, one needs to further postulate that all asymptotic states are colourless, i.e., singlets under rotations in colour space. This assumption is known as the confinement hypothesis, because it implies the non-observability of free quarks: since quarks carry colour they are confined within colour-singlet bound states.

A direct test of the colour quantum number can be obtained from the ratio

$$
R_{e^{+} e^{-}} \equiv \frac{\sigma\left(e^{+} e^{-} \rightarrow \text { hadrons }\right)}{\sigma\left(e^{+} e^{-} \rightarrow \mu^{+} \mu^{-}\right)} .
$$

The hadronic production occurs through $e^{+} e^{-} \rightarrow \gamma^{*}, Z^{*} \rightarrow q \bar{q} \rightarrow$ hadrons (Fig. 2). Since quarks are assumed to be confined, the probability to hadronize is just one; therefore, summing over all possible quarks in the final state, we can estimate the inclusive cross-section into hadrons. The electroweak production factors which are common with the $e^{+} e^{-} \rightarrow \gamma^{*}, Z^{*} \rightarrow \mu^{+} \mu^{-}$process cancel in the ratio (17). At energies well below the $Z$ peak, the cross-section is dominated by the $\gamma$-exchange amplitude; the ratio $R_{e^{+} e^{-}}$is then given by the sum of the quark electric charges squared:

$$
R_{e^{+} e^{-}} \approx N_{C} \sum_{f=1}^{N_{f}} Q_{f}^{2}=\left\{\begin{array}{cc}
\frac{2}{3} N_{C}=2, & \left(N_{f}=3: u, d, s\right) \\
\frac{10}{9} N_{C}=\frac{10}{3}, & \left(N_{f}=4: u, d, s, c\right) \\
\frac{11}{9} N_{C}=\frac{11}{3}, & \left(N_{f}=5: u, d, s, c, b\right)
\end{array} .\right.
$$

This result involves an explicit sum over the $N_{f}$ quark flavours which are kinematically accessible $\left[4 m_{q}^{2}<s \equiv\left(p_{e^{-}}+p_{e^{+}}\right)^{2}\right.$ ], weighted by the number of different colour possibilities. The measured ratio is shown in Fig. 3 . Although the simple formula (18) cannot explain the complicated structure around the different quark thresholds, it gives the right average value of the cross-section (away from thresholds), provided that $N_{C}$ is taken to be three. The agreement is better at larger energies. Notice that strong interactions have not been taken into account; only the confinement hypothesis has been used.

Electromagnetic interactions are associated with the fermion electric charges, while the quark flavours (up, down, strange, charm, bottom, top) are related to electroweak phenomena. The strong forces are flavour conserving and flavour independent. On the other side, the carriers of the electroweak interaction $\left(\gamma, Z, W^{ \pm}\right)$do not couple to the quark colour. Thus it seems natural to take colour as the charge associated with the strong forces and try to build a quantum field theory based on it [21,22]. 


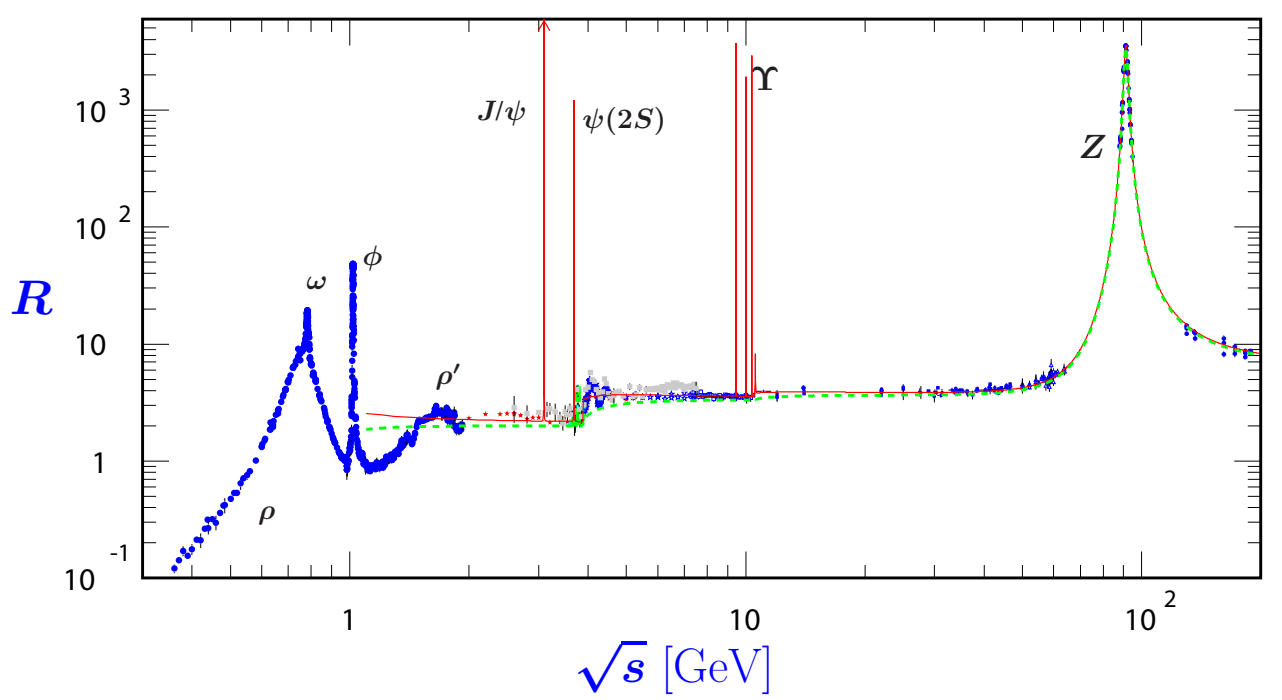

Fig. 3: World data on the ratio $R_{e^{+} e^{-}}$[9]. The broken lines show the naive quark model approximation with $N_{C}=3$. The solid curve is the 3-loop perturbative QCD prediction.

\subsubsection{Non-Abelian gauge symmetry}

Let us denote $q_{f}^{\alpha}$ a quark field of colour $\alpha$ and flavour $f$. To simplify the equations, let us adopt a vector notation in colour space: $q_{f}^{T} \equiv\left(q_{f}^{1}, q_{f}^{2}, q_{f}^{3}\right)$. The free Lagrangian

$$
\mathcal{L}_{0}=\sum_{f} \bar{q}_{f}\left(i \gamma^{\mu} \partial_{\mu}-m_{f}\right) q_{f}
$$

is invariant under arbitrary global $S U(3)_{C}$ transformations in colour space,

$$
q_{f}^{\alpha} \longrightarrow\left(q_{f}^{\alpha}\right)^{\prime}=U_{\beta}^{\alpha} q_{f}^{\beta}, \quad U U^{\dagger}=U^{\dagger} U=1, \quad \operatorname{det} U=1 .
$$

The $S U(3)_{C}$ matrices can be written in the form

$$
U=\exp \left\{i \frac{\lambda^{a}}{2} \theta_{a}\right\}
$$

where $\frac{1}{2} \lambda^{a}(a=1,2, \ldots, 8)$ denote the generators of the fundamental representation of the $S U(3)_{C}$ algebra, $\theta_{a}$ are arbitrary parameters and a sum over repeated colour indices is understood. The matrices $\lambda^{a}$ are traceless and satisfy the commutation relations

$$
\left[\frac{\lambda^{a}}{2}, \frac{\lambda^{b}}{2}\right]=i f^{a b c} \frac{\lambda^{c}}{2}
$$

with $f^{a b c}$ the $S U(3)_{C}$ structure constants, which are real and totally antisymmetric. Some useful properties of $S U(N)$ matrices are collected in Appendix B.

As in the QED case, we can now require the Lagrangian to be also invariant under local $S U(3)_{C}$ transformations, $\theta_{a}=\theta_{a}(x)$. To satisfy this requirement, we need to change the quark derivatives by covariant objects. Since we have now eight independent gauge parameters, eight different gauge bosons $G_{a}^{\mu}(x)$, the so-called gluons, are needed:

$$
D^{\mu} q_{f} \equiv\left[\partial^{\mu}+i g_{s} \frac{\lambda^{a}}{2} G_{a}^{\mu}(x)\right] q_{f} \equiv\left[\partial^{\mu}+i g_{s} G^{\mu}(x)\right] q_{f} .
$$



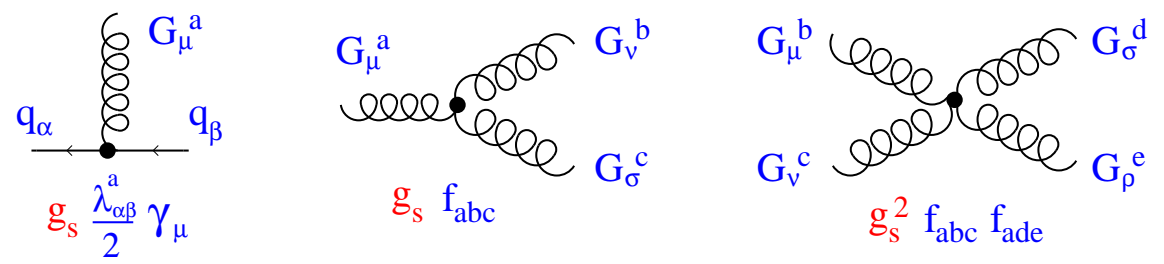

Fig. 4: Interaction vertices of the QCD Lagrangian.

Notice that we have introduced the compact matrix notation

$$
\left[G^{\mu}(x)\right]_{\alpha \beta} \equiv\left(\frac{\lambda^{a}}{2}\right)_{\alpha \beta} G_{a}^{\mu}(x)
$$

and a colour identity matrix is implicit in the derivative term. We want $D^{\mu} q_{f}$ to transform in exactly the same way as the colour-vector $q_{f}$; this fixes the transformation properties of the gauge fields:

$$
D^{\mu} \longrightarrow\left(D^{\mu}\right)^{\prime}=U D^{\mu} U^{\dagger}, \quad G^{\mu} \longrightarrow\left(G^{\mu}\right)^{\prime}=U G^{\mu} U^{\dagger}+\frac{i}{g_{s}}\left(\partial^{\mu} U\right) U^{\dagger} .
$$

Under an infinitesimal $S U(3)_{C}$ transformation,

$$
\begin{aligned}
q_{f}^{\alpha} & \longrightarrow\left(q_{f}^{\alpha}\right)^{\prime}=q_{f}^{\alpha}+i\left(\frac{\lambda^{a}}{2}\right)_{\alpha \beta} \delta \theta_{a} q_{f}^{\beta}, \\
G_{a}^{\mu} \longrightarrow\left(G_{a}^{\mu}\right)^{\prime} & =G_{a}^{\mu}-\frac{1}{g_{s}} \partial^{\mu}\left(\delta \theta_{a}\right)-f^{a b c} \delta \theta_{b} G_{c}^{\mu} .
\end{aligned}
$$

The gauge transformation of the gluon fields is more complicated than the one obtained in QED for the photon. The non-commutativity of the $S U(3)_{C}$ matrices gives rise to an additional term involving the gluon fields themselves. For constant $\delta \theta_{a}$, the transformation rule for the gauge fields is expressed in terms of the structure constants $f^{a b c}$; thus, the gluon fields belong to the adjoint representation of the colour group (see Appendix B). Note also that there is a unique $S U(3)_{C}$ coupling $g_{s}$. In QED it was possible to assign arbitrary electromagnetic charges to the different fermions. Since the commutation relation (22) is non-linear, this freedom does not exist for $S U(3)_{C}$. All colour-triplet quark flavours couple to the gluon fields with exactly the same interaction strength.

To build a gauge-invariant kinetic term for the gluon fields, we introduce the corresponding field strengths:

$$
\begin{aligned}
& G^{\mu \nu}(x) \equiv-\frac{i}{g_{s}}\left[D^{\mu}, D^{\nu}\right]=\partial^{\mu} G^{\nu}-\partial^{\nu} G^{\mu}+i g_{s}\left[G^{\mu}, G^{\nu}\right] \equiv \frac{\lambda^{a}}{2} G_{a}^{\mu \nu}(x), \\
& G_{a}^{\mu \nu}(x)=\partial^{\mu} G_{a}^{\nu}-\partial^{\nu} G_{a}^{\mu}-g_{s} f^{a b c} G_{b}^{\mu} G_{c}^{\nu}
\end{aligned}
$$

Under a $S U(3)_{C}$ gauge transformation,

$$
G^{\mu \nu} \longrightarrow\left(G^{\mu \nu}\right)^{\prime}=U G^{\mu \nu} U^{\dagger}
$$

and the colour trace $\operatorname{Tr}\left(G^{\mu \nu} G_{\mu \nu}\right)=\frac{1}{2} G_{a}^{\mu \nu} G_{\mu \nu}^{a}$ remains invariant. Taking the proper normalization for the gluon kinetic term, we finally have the $S U(3)_{C}$ invariant Lagrangian of Quantum Chromodynamics (QCD):

$$
\mathcal{L}_{\mathrm{QCD}} \equiv-\frac{1}{4} G_{a}^{\mu \nu} G_{\mu \nu}^{a}+\sum_{f} \bar{q}_{f}\left(i \gamma^{\mu} D_{\mu}-m_{f}\right) q_{f}
$$



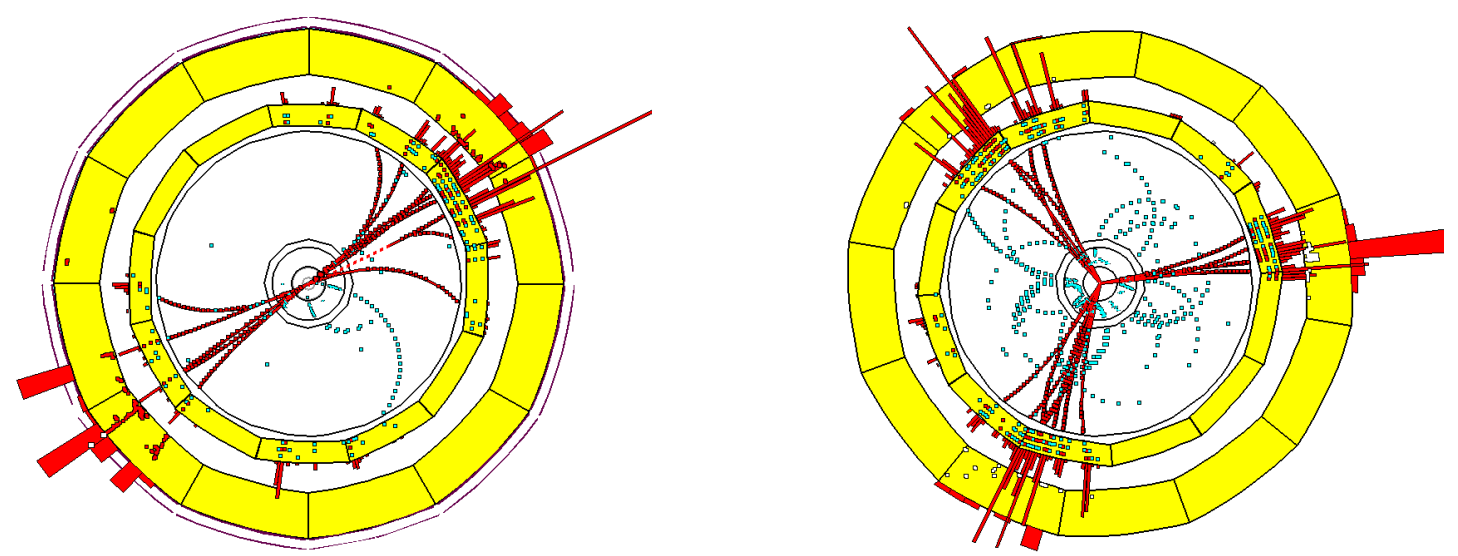

Fig. 5: Two- and three-jet events from the hadronic $Z$ boson decays $Z \rightarrow q \bar{q}$ and $Z \rightarrow q \bar{q} G$ (ALEPH) [23].

The $S U(3)_{C}$ gauge symmetry forbids to add a mass term for the gluon fields, $\frac{1}{2} m_{G}^{2} G_{a}^{\mu} G_{\mu}^{a}$, because it is not invariant under the transformation (25). The gauge bosons are, therefore, massless spin-1 particles.

It is worth while to decompose the Lagrangian into its different pieces:

$$
\begin{aligned}
\mathcal{L}_{\mathrm{QCD}}= & -\frac{1}{4}\left(\partial^{\mu} G_{a}^{\nu}-\partial^{\nu} G_{a}^{\mu}\right)\left(\partial_{\mu} G_{\nu}^{a}-\partial_{\nu} G_{\mu}^{a}\right)+\sum_{f} \bar{q}_{f}^{\alpha}\left(i \gamma^{\mu} \partial_{\mu}-m_{f}\right) q_{f}^{\alpha} \\
& -g_{s} G_{a}^{\mu} \sum_{f} \bar{q}_{f}^{\alpha} \gamma_{\mu}\left(\frac{\lambda^{a}}{2}\right)_{\alpha \beta} q_{f}^{\beta} \\
& +\frac{g_{s}}{2} f^{a b c}\left(\partial^{\mu} G_{a}^{\nu}-\partial^{\nu} G_{a}^{\mu}\right) G_{\mu}^{b} G_{\nu}^{c}-\frac{g_{s}^{2}}{4} f^{a b c} f_{a d e} G_{b}^{\mu} G_{c}^{\nu} G_{\mu}^{d} G_{\nu}^{e} .
\end{aligned}
$$

The first line contains the correct (quadratic) kinetic terms for the different fields, which give rise to the corresponding propagators. The colour interaction between quarks and gluons is given by the second line; it involves the $S U(3)_{C}$ matrices $\lambda^{a}$. Finally, owing to the non-Abelian character of the colour group, the $G_{a}^{\mu \nu} G_{\mu \nu}^{a}$ term generates the cubic and quartic gluon self-interactions shown in the last line; the strength of these interactions (Fig. 4) is given by the same coupling $g_{s}$ which appears in the fermionic piece of the Lagrangian.

In spite of the rich physics contained in it, the Lagrangian (29) looks very simple because of its colour symmetry properties. All interactions are given in terms of a single universal coupling $g_{s}$, which is called the strong coupling constant. The existence of self-interactions among the gauge fields is a new feature that was not present in QED; it seems then reasonable to expect that these gauge self-interactions could explain properties like asymptotic freedom (strong interactions become weaker at short distances) and confinement (the strong forces increase at large distances), which do not appear in QED [6].

Without any detailed calculation, one can already extract qualitative physical consequences from $\mathcal{L}_{\mathrm{QCD}}$. Quarks can emit gluons. At lowest order in $g_{s}$, the dominant process will be the emission of a single gauge boson; thus, the hadronic decay of the $Z$ should result in some $Z \rightarrow q \bar{q} G$ events, in addition to the dominant $Z \rightarrow q \bar{q}$ decays. Figure 5 clearly shows that 3 -jet events, with the required kinematics, indeed appear in the LEP data. Similar events show up in $e^{+} e^{-}$annihilation into hadrons, away from the $Z$ peak. The ratio between 3 -jet and 2-jet events provides a simple estimate of the strength of the strong interaction at LEP energies $\left(s=M_{Z}^{2}\right): \alpha_{s} \equiv g_{s}^{2} /(4 \pi) \sim 0.12$. 


\section{Electroweak Unification}

\subsection{Experimental facts}

Low-energy experiments have provided a large amount of information about the dynamics underlying flavour-changing processes. The detailed analysis of the energy and angular distributions in $\beta$ decays, such as $\mu^{-} \rightarrow e^{-} \bar{\nu}_{e} \nu_{\mu}$ or $n \rightarrow p e^{-} \bar{\nu}_{e}$, made clear that only the left-handed (right-handed) fermion (antifermion) chiralities participate in those weak transitions; moreover, the strength of the interaction appears to be universal. This is further corroborated through the study of other processes like $\pi^{-} \rightarrow$ $e^{-} \bar{\nu}_{e}$ or $\pi^{-} \rightarrow \mu^{-} \bar{\nu}_{\mu}$, which show that neutrinos have left-handed chiralities while anti-neutrinos are right-handed.

From neutrino scattering data, we learnt the existence of different neutrino types $\left(\nu_{e} \neq \nu_{\mu}\right)$ and that there are separately conserved lepton quantum numbers which distinguish neutrinos from antineutrinos; thus we observe the transitions $\bar{\nu}_{e} p \rightarrow e^{+} n, \nu_{e} n \rightarrow e^{-} p, \bar{\nu}_{\mu} p \rightarrow \mu^{+} n$ or $\nu_{\mu} n \rightarrow \mu^{-} p$, but we do not see processes like $\nu_{e} p \not \rightarrow e^{+} n, \bar{\nu}_{e} n \not \rightarrow e^{-} p, \bar{\nu}_{\mu} p \not \rightarrow e^{+} n$ or $\nu_{\mu} n \not \rightarrow e^{-} p$.

Together with theoretical considerations related to unitarity (a proper high-energy behaviour) and the absence of flavour-changing neutral-current transitions $\left(\mu^{-} \not \rightarrow e^{-} e^{-} e^{+}, s \not \rightarrow d \ell^{+} \ell^{-}\right)$, the lowenergy information was good enough to determine the structure of the modern electroweak theory [24]. The intermediate vector bosons $W^{ \pm}$and $Z$ were theoretically introduced and their masses correctly estimated, before their experimental discovery. Nowadays, we have accumulated huge numbers of $W^{ \pm}$ and $Z$ decay events, which bring much direct experimental evidence of their dynamical properties.

\subsubsection{Charged currents}
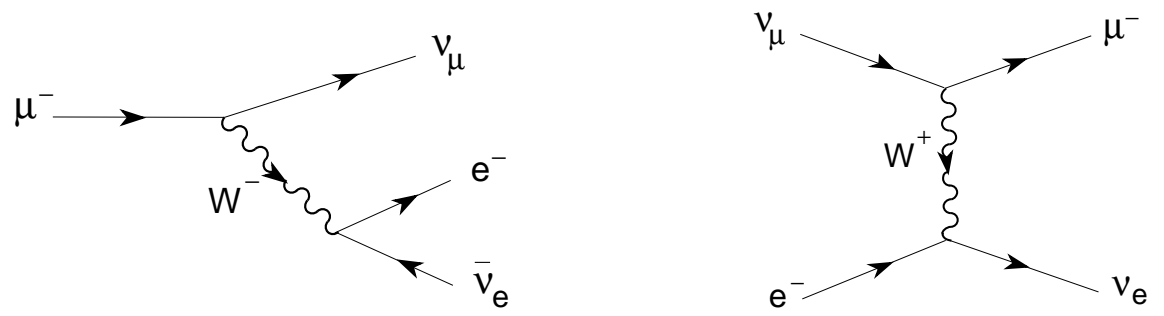

Fig. 6: Tree-level Feynman diagrams for $\mu^{-} \rightarrow e^{-} \bar{\nu}_{e} \nu_{\mu}$ and $\nu_{\mu} e^{-} \rightarrow \mu^{-} \nu_{e}$.

The interaction of quarks and leptons with the $W^{ \pm}$bosons (Fig. 6) exhibits the following features:

- Only left-handed fermions and right-handed antifermions couple to the $W^{ \pm}$. Therefore, there is a $100 \%$ breaking of parity ( $\mathcal{P}$ : left $\leftrightarrow$ right) and charge conjugation $(\mathcal{C}$ : particle $\leftrightarrow$ antiparticle). However, the combined transformation $\mathcal{C P}$ is still a good symmetry.

- The $W^{ \pm}$bosons couple to the fermionic doublets in Eq. (2), where the electric charges of the two fermion partners differ in one unit. The decay channels of the $W^{-}$are then:

$$
W^{-} \rightarrow e^{-} \bar{\nu}_{e}, \mu^{-} \bar{\nu}_{\mu}, \tau^{-} \bar{\nu}_{\tau}, d^{\prime} \bar{u}, s^{\prime} \bar{c} .
$$

Owing to the very high mass of the top quark [25], $m_{t}=173 \mathrm{GeV}>M_{W}=80.4 \mathrm{GeV}$, its on-shell production through $W^{-} \rightarrow b^{\prime} \bar{t}$ is kinematically forbidden.

- All fermion doublets couple to the $W^{ \pm}$bosons with the same universal strength.

- The doublet partners of the up, charm and top quarks appear to be mixtures of the three quarks with charge $-\frac{1}{3}$ :

$$
\left(\begin{array}{c}
d^{\prime} \\
s^{\prime} \\
b^{\prime}
\end{array}\right)=\mathbf{V}\left(\begin{array}{c}
d \\
s \\
b
\end{array}\right), \quad \quad \quad \mathbf{V}^{\dagger}=\mathbf{V}^{\dagger} \mathbf{V}=1
$$



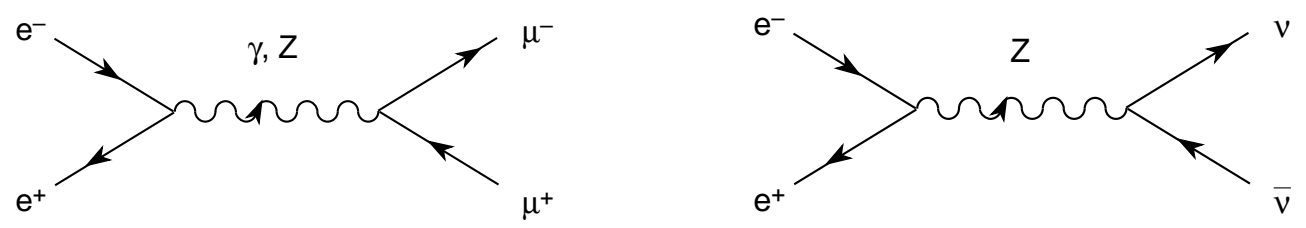

Fig. 7: Tree-level Feynman diagrams for $e^{+} e^{-} \rightarrow \mu^{+} \mu^{-}$and $e^{+} e^{-} \rightarrow \nu \bar{\nu}$.

Thus, the weak eigenstates $d^{\prime}, s^{\prime}, b^{\prime}$ are different than the mass eigenstates $d, s, b$. They are related through the $3 \times 3$ unitary matrix $\mathbf{V}$, which characterizes flavour-mixing phenomena.

- The experimental evidence of neutrino oscillations shows that $\nu_{e}, \nu_{\mu}$ and $\nu_{\tau}$ are also mixtures of mass eigenstates. However, the neutrino masses are tiny: $\left|m_{\nu_{3}}^{2}-m_{\nu_{2}}^{2}\right| \sim 2.4 \cdot 10^{-3} \mathrm{eV}^{2}$, $m_{\nu_{2}}^{2}-m_{\nu_{1}}^{2} \sim 7.6 \cdot 10^{-5} \mathrm{eV}^{2}[\overline{9]}$.

\subsubsection{Neutral currents}

The neutral carriers of the electromagnetic and weak interactions have fermionic couplings (Fig. 7) with the following properties:

- All interacting vertices are flavour conserving. Both the $\gamma$ and the $Z$ couple to a fermion and its own antifermion, i.e., $\gamma f \bar{f}$ and $Z f \bar{f}$. Transitions of the type $\mu \not \rightarrow e \gamma$ or $Z \not \rightarrow e^{ \pm} \mu^{\mp}$ have never been observed.

- The interactions depend on the fermion electric charge $Q_{f}$. Fermions with the same $Q_{f}$ have exactly the same universal couplings. Neutrinos do not have electromagnetic interactions $\left(Q_{\nu}=\right.$ 0 ), but they have a non-zero coupling to the $Z$ boson.

- Photons have the same interaction for both fermion chiralities, but the $Z$ couplings are different for left-handed and right-handed fermions. The neutrino coupling to the $Z$ involves only left-handed chiralities.

- There are three different light neutrino species.

\subsection{The $\mathrm{SU}(2)_{\mathrm{L}} \otimes \mathrm{U}(1)_{\mathrm{Y}}$ theory}

Using gauge invariance, we have been able to determine the right QED and QCD Lagrangians. To describe weak interactions, we need a more elaborated structure, with several fermionic flavours and different properties for left- and right-handed fields; moreover, the left-handed fermions should appear in doublets, and we would like to have massive gauge bosons $W^{ \pm}$and $Z$ in addition to the photon. The simplest group with doublet representations is $S U(2)$. We want to include also the electromagnetic interactions; thus we need an additional $U(1)$ group. The obvious symmetry group to consider is then

$$
G \equiv S U(2)_{L} \otimes U(1)_{Y},
$$

where $L$ refers to left-handed fields. We do not specify, for the moment, the meaning of the subindex $Y$ since, as we will see, the naive identification with electromagnetism does not work.

For simplicity, let us consider a single family of quarks, and introduce the notation

$$
\psi_{1}(x)=\left(\begin{array}{l}
u \\
d
\end{array}\right)_{L}, \quad \psi_{2}(x)=u_{R}, \quad \psi_{3}(x)=d_{R} .
$$

Our discussion will also be valid for the lepton sector, with the identification

$$
\psi_{1}(x)=\left(\begin{array}{c}
\nu_{e} \\
e^{-}
\end{array}\right)_{L}, \quad \psi_{2}(x)=\nu_{e R}, \quad \psi_{3}(x)=e_{R}^{-} .
$$


As in the QED and QCD cases, let us consider the free Lagrangian

$$
\mathcal{L}_{0}=i \bar{u}(x) \gamma^{\mu} \partial_{\mu} u(x)+i \bar{d}(x) \gamma^{\mu} \partial_{\mu} d(x)=\sum_{j=1}^{3} i \bar{\psi}_{j}(x) \gamma^{\mu} \partial_{\mu} \psi_{j}(x) .
$$

$\mathcal{L}_{0}$ is invariant under global $G$ transformations in flavour space:

$$
\begin{aligned}
& \psi_{1}(x) \quad \stackrel{G}{\rightarrow} \quad \psi_{1}^{\prime}(x) \equiv \exp \left\{i y_{1} \beta\right\} U_{L} \psi_{1}(x), \\
& \psi_{2}(x) \quad \longrightarrow \quad \psi_{2}^{\prime}(x) \equiv \exp \left\{i y_{2} \beta\right\} \psi_{2}(x), \\
& \psi_{3}(x) \quad \rightarrow \quad \psi_{3}^{\prime}(x) \equiv \exp \left\{i y_{3} \beta\right\} \psi_{3}(x),
\end{aligned}
$$

where the $S U(2)_{L}$ transformation [ $\sigma_{i}$ are the Pauli matrices $[$ B.3) $]$

$$
U_{L} \equiv \exp \left\{i \frac{\sigma_{i}}{2} \alpha^{i}\right\} \quad(i=1,2,3)
$$

only acts on the doublet field $\psi_{1}$. The parameters $y_{i}$ are called hypercharges, since the $U(1)_{Y}$ phase transformation is analogous to the QED one. The matrix transformation $U_{L}$ is non-Abelian as in QCD. Notice that we have not included a mass term in Eq. (36) because it would mix the left- and right-handed fields [see Eq. A.17] ], therefore spoiling our symmetry considerations.

We can now require the Lagrangian to be also invariant under local $S U(2)_{L} \otimes U(1)_{Y}$ gauge transformations, i.e., with $\alpha^{i}=\alpha^{i}(x)$ and $\beta=\beta(x)$. In order to satisfy this symmetry requirement, we need to change the fermion derivatives by covariant objects. Since we have now four gauge parameters, $\alpha^{i}(x)$ and $\beta(x)$, four different gauge bosons are needed:

$$
\begin{aligned}
D_{\mu} \psi_{1}(x) & \equiv\left[\partial_{\mu}+i g \widetilde{W}_{\mu}(x)+i g^{\prime} y_{1} B_{\mu}(x)\right] \psi_{1}(x), \\
D_{\mu} \psi_{2}(x) & \equiv\left[\partial_{\mu}+i g^{\prime} y_{2} B_{\mu}(x)\right] \psi_{2}(x) \\
D_{\mu} \psi_{3}(x) & \equiv\left[\partial_{\mu}+i g^{\prime} y_{3} B_{\mu}(x)\right] \psi_{3}(x)
\end{aligned}
$$

where

$$
\widetilde{W}_{\mu}(x) \equiv \frac{\sigma_{i}}{2} W_{\mu}^{i}(x)
$$

denotes a $S U(2)_{L}$ matrix field. Thus we have the correct number of gauge fields to describe the $W^{ \pm}, Z$ and $\gamma$.

We want $D_{\mu} \psi_{j}(x)$ to transform in exactly the same way as the $\psi_{j}(x)$ fields; this fixes the transformation properties of the gauge fields:

$$
\begin{aligned}
B_{\mu}(x) & \stackrel{G}{\longrightarrow} \quad B_{\mu}^{\prime}(x) \equiv B_{\mu}(x)-\frac{1}{g^{\prime}} \partial_{\mu} \beta(x), \\
\widetilde{W}_{\mu} & \stackrel{G}{\longrightarrow} \widetilde{W}_{\mu}^{\prime} \equiv U_{L}(x) \widetilde{W}_{\mu} U_{L}^{\dagger}(x)+\frac{i}{g} \partial_{\mu} U_{L}(x) U_{L}^{\dagger}(x),
\end{aligned}
$$

where $U_{L}(x) \equiv \exp \left\{i \frac{\sigma_{i}}{2} \alpha^{i}(x)\right\}$. The transformation of $B_{\mu}$ is identical to the one obtained in QED for the photon, while the $S U(2)_{L} W_{\mu}^{i}$ fields transform in a way analogous to the gluon fields of QCD. Note that the $\psi_{j}$ couplings to $B_{\mu}$ are completely free as in QED, i.e., the hypercharges $y_{j}$ can be arbitrary parameters. Since the $S U(2)_{L}$ commutation relation is non-linear, this freedom does not exist for the $W_{\mu}^{i}$ : there is only a unique $S U(2)_{L}$ coupling $g$.

The Lagrangian

$$
\mathcal{L}=\sum_{j=1}^{3} i \bar{\psi}_{j}(x) \gamma^{\mu} D_{\mu} \psi_{j}(x)
$$


is invariant under local $G$ transformations. In order to build the gauge-invariant kinetic term for the gauge fields, we introduce the corresponding field strengths:

$$
\begin{gathered}
B_{\mu \nu} \equiv \partial_{\mu} B_{\nu}-\partial_{\nu} B_{\mu}, \\
\widetilde{W}_{\mu \nu} \equiv-\frac{i}{g}\left[\left(\partial_{\mu}+i g \widetilde{W}_{\mu}\right),\left(\partial_{\nu}+i g \widetilde{W}_{\nu}\right)\right]=\partial_{\mu} \widetilde{W}_{\nu}-\partial_{\nu} \widetilde{W}_{\mu}+i g\left[\widetilde{W}_{\mu}, \widetilde{W}_{\nu}\right], \\
\widetilde{W}_{\mu \nu} \equiv \frac{\sigma_{i}}{2} W_{\mu \nu}^{i}, \quad W_{\mu \nu}^{i}=\partial_{\mu} W_{\nu}^{i}-\partial_{\nu} W_{\mu}^{i}-g \epsilon^{i j k} W_{\mu}^{j} W_{\nu}^{k} .
\end{gathered}
$$

$B_{\mu \nu}$ remains invariant under $G$ transformations, while $\widetilde{W}_{\mu \nu}$ transforms covariantly:

$$
B_{\mu \nu} \stackrel{G}{\longrightarrow} B_{\mu \nu}, \quad \widetilde{W}_{\mu \nu} \stackrel{G}{\longrightarrow} U_{L} \widetilde{W}_{\mu \nu} U_{L}^{\dagger} .
$$

Therefore, the properly normalized kinetic Lagrangian is given by

$$
\mathcal{L}_{\text {Kin }}=-\frac{1}{4} B_{\mu \nu} B^{\mu \nu}-\frac{1}{2} \operatorname{Tr}\left[\widetilde{W}_{\mu \nu} \widetilde{W}^{\mu \nu}\right]=-\frac{1}{4} B_{\mu \nu} B^{\mu \nu}-\frac{1}{4} W_{\mu \nu}^{i} W_{i}^{\mu \nu} .
$$

Since the field strengths $W_{\mu \nu}^{i}$ contain a quadratic piece, the Lagrangian $\mathcal{L}_{\text {Kin }}$ gives rise to cubic and quartic self-interactions among the gauge fields. The strength of these interactions is given by the same $S U(2)_{L}$ coupling $g$ which appears in the fermionic piece of the Lagrangian.

The gauge symmetry forbids the writing of a mass term for the gauge bosons. Fermionic masses are also not possible, because they would communicate the left- and right-handed fields, which have different transformation properties, and therefore would produce an explicit breaking of the gauge symmetry. Thus, the $S U(2)_{L} \otimes U(1)_{Y}$ Lagrangian in Eqs. (43) and (48) only contains massless fields.

\subsection{Charged-current interaction}
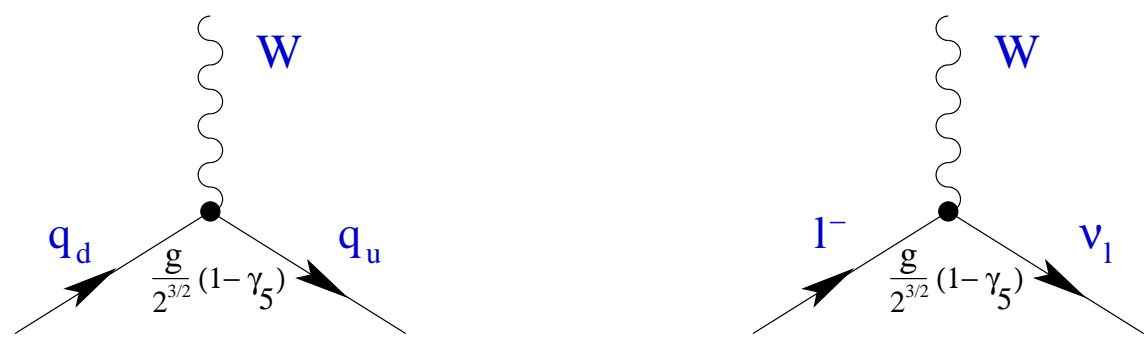

Fig. 8: Charged-current interaction vertices.

The Lagrangian (43) contains interactions of the fermion fields with the gauge bosons,

$$
\mathcal{L} \longrightarrow-g \bar{\psi}_{1} \gamma^{\mu} \widetilde{W}_{\mu} \psi_{1}-g^{\prime} B_{\mu} \sum_{j=1}^{3} y_{j} \bar{\psi}_{j} \gamma^{\mu} \psi_{j} .
$$

The term containing the $S U(2)_{L}$ matrix

$$
\widetilde{W}_{\mu}=\frac{\sigma^{i}}{2} W_{\mu}^{i}=\frac{1}{2}\left(\begin{array}{cc}
W_{\mu}^{3} & \sqrt{2} W_{\mu}^{\dagger} \\
\sqrt{2} W_{\mu} & -W_{\mu}^{3}
\end{array}\right)
$$

gives rise to charged-current interactions with the boson field $W_{\mu} \equiv\left(W_{\mu}^{1}+i W_{\mu}^{2}\right) / \sqrt{2}$ and its complexconjugate $W_{\mu}^{\dagger} \equiv\left(W_{\mu}^{1}-i W_{\mu}^{2}\right) / \sqrt{2}$ (Fig. 8). For a single family of quarks and leptons,

$$
\mathcal{L}_{\mathrm{CC}}=-\frac{g}{2 \sqrt{2}}\left\{W_{\mu}^{\dagger}\left[\bar{u} \gamma^{\mu}\left(1-\gamma_{5}\right) d+\bar{\nu}_{e} \gamma^{\mu}\left(1-\gamma_{5}\right) e\right]+\text { h.c. }\right\} \text {. }
$$

The universality of the quark and lepton interactions is now a direct consequence of the assumed gauge symmetry. Note, however, that Eq. (51) cannot describe the observed dynamics, because the gauge bosons are massless and, therefore, give rise to long-range forces. 


\subsection{Neutral-current interaction}
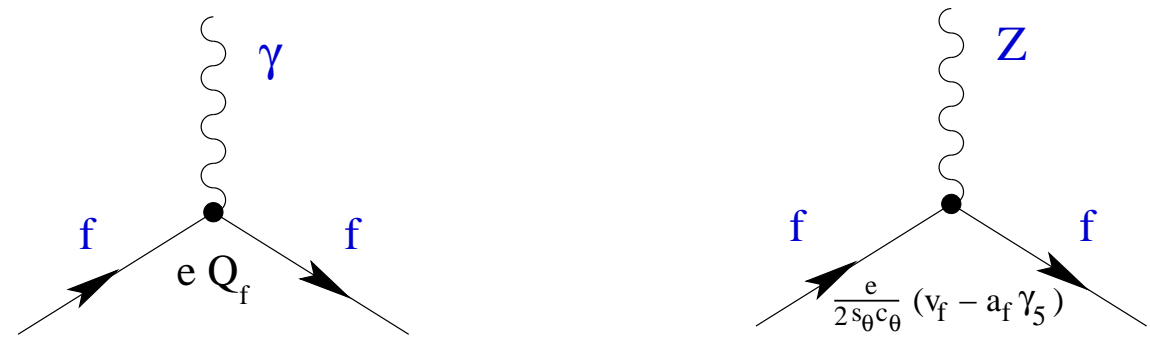

Fig. 9: Neutral-current interaction vertices $\left(s_{\theta} \equiv \sin \theta_{W}, c_{\theta} \equiv \cos \theta_{W}\right)$.

Equation (49) contains also interactions with the neutral gauge fields $W_{\mu}^{3}$ and $B_{\mu}$. We would like to identify these bosons with the $Z$ and the $\gamma$. However, since the photon has the same interaction with both fermion chiralities, the singlet gauge boson $B_{\mu}$ cannot be equal to the electromagnetic field. That would require $y_{1}=y_{2}=y_{3}$ and $g^{\prime} y_{j}=e Q_{j}$, which cannot be simultaneously true.

Since both fields are neutral, we can try with an arbitrary combination of them:

$$
\left(\begin{array}{c}
W_{\mu}^{3} \\
B_{\mu}
\end{array}\right) \equiv\left(\begin{array}{cc}
\cos \theta_{W} & \sin \theta_{W} \\
-\sin \theta_{W} & \cos \theta_{W}
\end{array}\right)\left(\begin{array}{c}
Z_{\mu} \\
A_{\mu}
\end{array}\right)
$$

The physical $Z$ boson has a mass different from zero, which is forbidden by the local gauge symmetry. We will see in the next section how it is possible to generate non-zero boson masses, through the SSB mechanism. For the moment, we just assume that something breaks the symmetry, generating the $Z$ mass, and that the neutral mass eigenstates are a mixture of the triplet and singlet $S U(2)_{L}$ fields. In terms of the fields $Z$ and $\gamma$, the neutral-current Lagrangian is given by

$$
\mathcal{L}_{\mathrm{NC}}=-\sum_{j} \bar{\psi}_{j} \gamma^{\mu}\left\{A_{\mu}\left[g \frac{\sigma_{3}}{2} \sin \theta_{W}+g^{\prime} y_{j} \cos \theta_{W}\right]+Z_{\mu}\left[g \frac{\sigma_{3}}{2} \cos \theta_{W}-g^{\prime} y_{j} \sin \theta_{W}\right]\right\} \psi_{j}
$$

In order to get QED from the $A_{\mu}$ piece, one needs to impose the conditions:

$$
g \sin \theta_{W}=g^{\prime} \cos \theta_{W}=e, \quad Y=Q-T_{3},
$$

where $T_{3} \equiv \sigma_{3} / 2$ and $Q$ denotes the electromagnetic charge operator

$$
Q_{1} \equiv\left(\begin{array}{cc}
Q_{u / \nu} & 0 \\
0 & Q_{d / e}
\end{array}\right), \quad Q_{2}=Q_{u / \nu}, \quad Q_{3}=Q_{d / e} .
$$

The first equality relates the $S U(2)_{L}$ and $U(1)_{Y}$ couplings to the electromagnetic coupling, providing the wanted unification of the electroweak interactions. The second identity fixes the fermion hypercharges in terms of their electric charge and weak isospin quantum numbers:

$$
\begin{aligned}
& \text { Quarks: } \quad y_{1}=Q_{u}-\frac{1}{2}=Q_{d}+\frac{1}{2}=\frac{1}{6}, \quad y_{2}=Q_{u}=\frac{2}{3}, \quad y_{3}=Q_{d}=-\frac{1}{3} \text {, } \\
& \text { Leptons: } y_{1}=Q_{\nu}-\frac{1}{2}=Q_{e}+\frac{1}{2}=-\frac{1}{2}, \quad y_{2}=Q_{\nu}=0, \quad y_{3}=Q_{e}=-1 .
\end{aligned}
$$

A hypothetical right-handed neutrino would have both electric charge and weak hypercharge equal to zero. Since it would not couple either to the $W^{ \pm}$bosons, such a particle would not have any kind of interaction (sterile neutrino). For aesthetic reasons, we shall then not consider right-handed neutrinos any longer.

Using the relations (54), the neutral-current Lagrangian can be written as

$$
\mathcal{L}_{\mathrm{NC}}=\mathcal{L}_{\mathrm{QED}}+\mathcal{L}_{\mathrm{NC}}^{Z}
$$


Table 1: Neutral-current couplings.

\begin{tabular}{ccccc}
\hline \hline & $u$ & $d$ & $\nu_{e}$ & $e$ \\
\hline $2 v_{f}$ & $1-\frac{8}{3} \sin ^{2} \theta_{W}$ & $-1+\frac{4}{3} \sin ^{2} \theta_{W}$ & 1 & $-1+4 \sin ^{2} \theta_{W}$ \\
$2 a_{f}$ & 1 & -1 & 1 & -1 \\
\hline \hline
\end{tabular}

where

$$
\mathcal{L}_{\mathrm{QED}}=-e A_{\mu} \sum_{j} \bar{\psi}_{j} \gamma^{\mu} Q_{j} \psi_{j} \equiv-e A_{\mu} J_{\mathrm{em}}^{\mu}
$$

is the usual QED Lagrangian and

$$
\mathcal{L}_{\mathrm{NC}}^{Z}=-\frac{e}{2 \sin \theta_{W} \cos \theta_{W}} J_{Z}^{\mu} Z_{\mu}
$$

contains the interaction of the $Z$ boson with the neutral fermionic current

$$
J_{Z}^{\mu} \equiv \sum_{j} \bar{\psi}_{j} \gamma^{\mu}\left(\sigma_{3}-2 \sin ^{2} \theta_{W} Q_{j}\right) \psi_{j}=J_{3}^{\mu}-2 \sin ^{2} \theta_{W} J_{\mathrm{em}}^{\mu} .
$$

In terms of the more usual fermion fields, $\mathcal{L}_{\mathrm{NC}}^{Z}$ has the form (Fig. 9)

$$
\mathcal{L}_{\mathrm{NC}}^{Z}=-\frac{e}{2 \sin \theta_{W} \cos \theta_{W}} Z_{\mu} \sum_{f} \bar{f} \gamma^{\mu}\left(v_{f}-a_{f} \gamma_{5}\right) f,
$$

where $a_{f}=T_{3}^{f}$ and $v_{f}=T_{3}^{f}\left(1-4\left|Q_{f}\right| \sin ^{2} \theta_{W}\right)$. Table1 1 shows the neutral-current couplings of the different fermions.

\subsection{Gauge self-interactions}
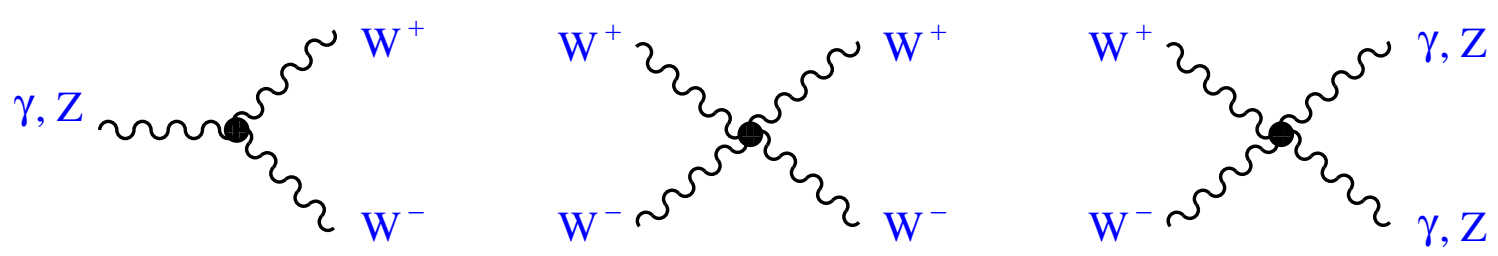

Fig. 10: Gauge boson self-interaction vertices.

In addition to the usual kinetic terms, the Lagrangian (48) generates cubic and quartic selfinteractions among the gauge bosons (Fig. 10):

$$
\begin{aligned}
\mathcal{L}_{3}= & i e \cot \theta_{W}\left\{\left(\partial^{\mu} W^{\nu}-\partial^{\nu} W^{\mu}\right) W_{\mu}^{\dagger} Z_{\nu}-\left(\partial^{\mu} W^{\nu \dagger}-\partial^{\nu} W^{\mu \dagger}\right) W_{\mu} Z_{\nu}+W_{\mu} W_{\nu}^{\dagger}\left(\partial^{\mu} Z^{\nu}-\partial^{\nu} Z^{\mu}\right)\right\} \\
& +i e\left\{\left(\partial^{\mu} W^{\nu}-\partial^{\nu} W^{\mu}\right) W_{\mu}^{\dagger} A_{\nu}-\left(\partial^{\mu} W^{\nu \dagger}-\partial^{\nu} W^{\mu \dagger}\right) W_{\mu} A_{\nu}+W_{\mu} W_{\nu}^{\dagger}\left(\partial^{\mu} A^{\nu}-\partial^{\nu} A^{\mu}\right)\right\} \\
\mathcal{L}_{4}= & -\frac{e^{2}}{2 \sin ^{2} \theta_{W}}\left\{\left(W_{\mu}^{\dagger} W^{\mu}\right)^{2}-W_{\mu}^{\dagger} W^{\mu \dagger} W_{\nu} W^{\nu}\right\}-e^{2} \cot ^{2} \theta_{W}\left\{W_{\mu}^{\dagger} W^{\mu} Z_{\nu} Z^{\nu}-W_{\mu}^{\dagger} Z^{\mu} W_{\nu} Z^{\nu}\right\}
\end{aligned}
$$




$$
\begin{aligned}
& -e^{2} \cot \theta_{W}\left\{2 W_{\mu}^{\dagger} W^{\mu} Z_{\nu} A^{\nu}-W_{\mu}^{\dagger} Z^{\mu} W_{\nu} A^{\nu}-W_{\mu}^{\dagger} A^{\mu} W_{\nu} Z^{\nu}\right\} \\
& -e^{2}\left\{W_{\mu}^{\dagger} W^{\mu} A_{\nu} A^{\nu}-W_{\mu}^{\dagger} A^{\mu} W_{\nu} A^{\nu}\right\} .
\end{aligned}
$$

Notice that at least a pair of charged $W$ bosons are always present. The $S U(2)_{L}$ algebra does not generate any neutral vertex with only photons and $Z$ bosons.

\section{Spontaneous Symmetry Breaking}

So far, we have been able to derive charged- and neutral-current interactions of the type needed to describe weak decays; we have nicely incorporated QED into the same theoretical framework and, moreover, we have got additional self-interactions of the gauge bosons, which are generated by the nonAbelian structure of the $S U(2)_{L}$ group. Gauge symmetry also guarantees that we have a well-defined renormalizable Lagrangian. However, this Lagrangian has very little to do with reality. Our gauge bosons are massless particles; while this is fine for the photon field, the physical $W^{ \pm}$and $Z$ bosons should be quite heavy objects.

In order to generate masses, we need to break the gauge symmetry in some way; however, we also need a fully symmetric Lagrangian to preserve renormalizability. This dilemma may be solved by the possibility of getting non-symmetric results from a symmetric Lagrangian.

Let us consider a Lagrangian, which

1. Is invariant under a group $G$ of transformations.

2. Has a degenerate set of states with minimal energy, which transform under $G$ as the members of a given multiplet.

If one of those states is arbitrarily selected as the ground state of the system, the symmetry is said to be spontaneously broken.

A well-known physical example is provided by a ferromagnet: although the Hamiltonian is invariant under rotations, the ground state has the electron spins aligned into some arbitrary direction; moreover, any higher-energy state, built from the ground state by a finite number of excitations, would share this anisotropy. In a quantum field theory, the ground state is the vacuum; thus the SSB mechanism will appear when there is a symmetric Lagrangian, but a non-symmetric vacuum.
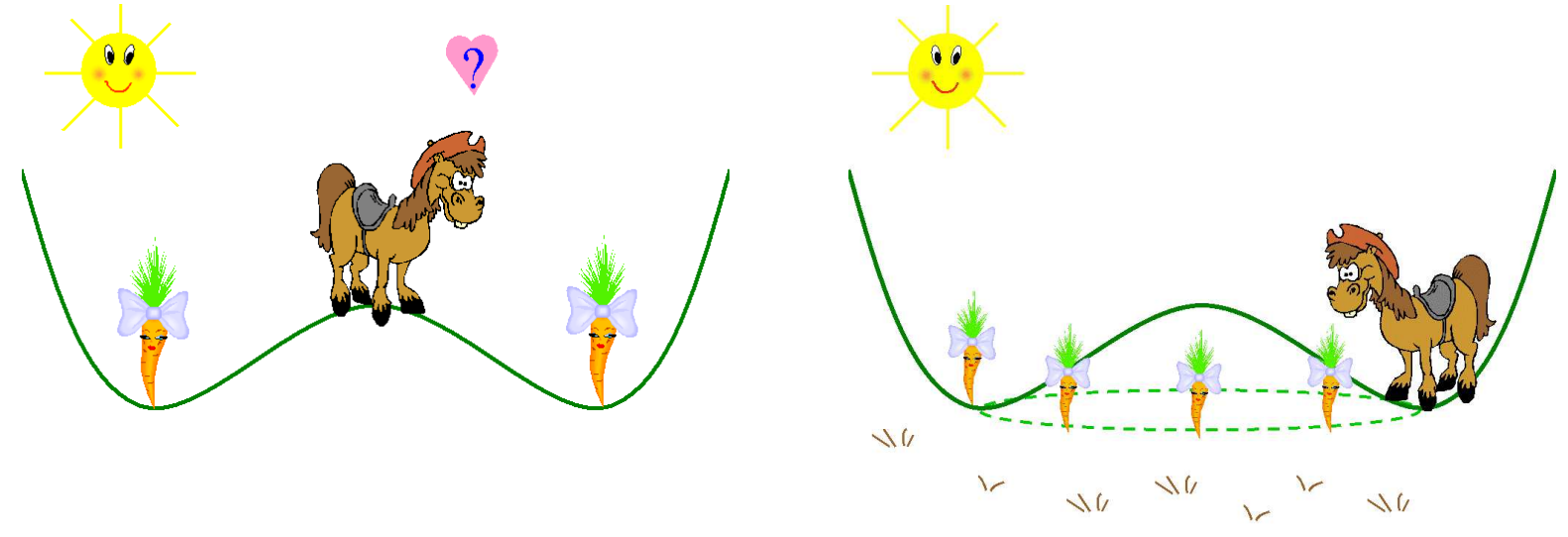

Fig. 11: Although Nicolás likes the symmetric food configuration, he must break the symmetry deciding which carrot is more appealing. In three dimensions, there is a continuous valley where Nicolás can move from one carrot to the next without effort. 
The horse in Fig. 11illustrates in a very simple way the phenomenon of SSB. Although the left and right carrots are identical, Nicolás must take a decision if he wants to get food. What is important is not whether he goes left or right, which are equivalent options, but that the symmetry gets broken. In two dimensions (discrete left-right symmetry), after eating the first carrot Nicolás would need to make an effort to climb the hill in order to reach the carrot on the other side; however, in three dimensions (continuous rotation symmetry) there is a marvelous flat circular valley along which Nicolás can move from one carrot to the next without any effort.

The existence of flat directions connecting the degenerate states of minimal energy is a general property of the SSB of continuous symmetries. In a quantum field theory it implies the existence of massless degrees of freedom.

\subsection{Goldstone theorem}
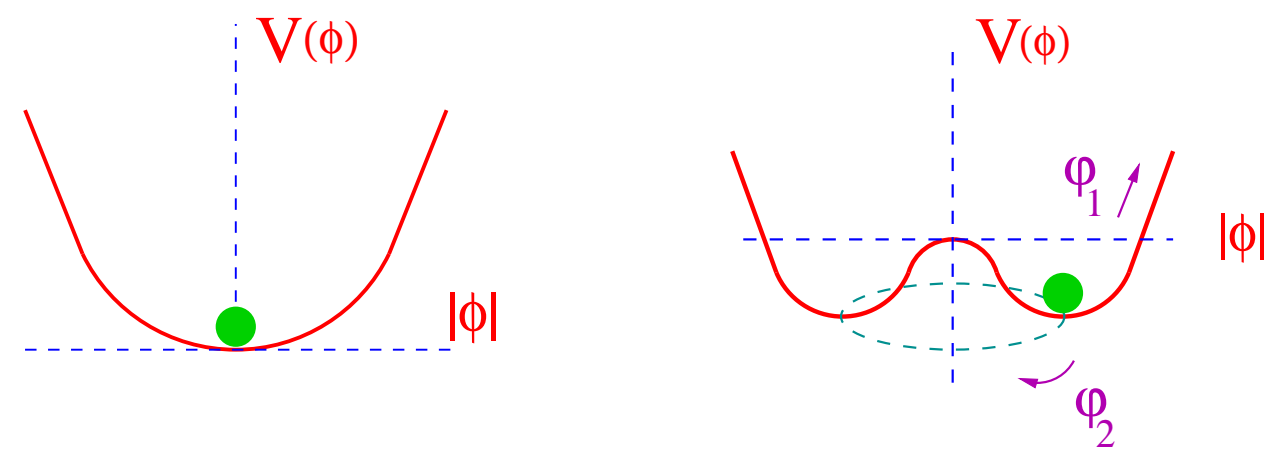

Fig. 12: Shape of the scalar potential for $\mu^{2}>0$ (left) and $\mu^{2}<0$ (right). In the second case there is a continuous set of degenerate vacua, corresponding to different phases $\theta$, connected through a massless field excitation $\varphi_{2}$.

Let us consider a complex scalar field $\phi(x)$, with Lagrangian

$$
\mathcal{L}=\partial_{\mu} \phi^{\dagger} \partial^{\mu} \phi-V(\phi), \quad V(\phi)=\mu^{2} \phi^{\dagger} \phi+h\left(\phi^{\dagger} \phi\right)^{2} .
$$

$\mathcal{L}$ is invariant under global phase transformations of the scalar field

$$
\phi(x) \longrightarrow \phi^{\prime}(x) \equiv \exp \{i \theta\} \phi(x) .
$$

In order to have a ground state the potential should be bounded from below, i.e., $h>0$. For the quadratic piece there are two possibilities, shown in Fig. 12.

1. $\boldsymbol{\mu}^{2}>0$ : The potential has only the trivial minimum $\phi=0$. It describes a massive scalar particle with mass $\mu$ and quartic coupling $h$.

2. $\boldsymbol{\mu}^{2}<0$ : The minimum is obtained for those field configurations satisfying

$$
\left|\phi_{0}\right|=\sqrt{\frac{-\mu^{2}}{2 h}} \equiv \frac{v}{\sqrt{2}}>0, \quad V\left(\phi_{0}\right)=-\frac{h}{4} v^{4} .
$$

Owing to the $U(1)$ phase invariance of the Lagrangian, there is an infinite number of degenerate states of minimum energy, $\phi_{0}(x)=\frac{v}{\sqrt{2}} \exp \{i \theta\}$. By choosing a particular solution, $\theta=0$ for example, as the ground state, the symmetry gets spontaneously broken. If we parametrize the excitations over the ground state as

$$
\phi(x) \equiv \frac{1}{\sqrt{2}}\left[v+\varphi_{1}(x)+i \varphi_{2}(x)\right],
$$


where $\varphi_{1}$ and $\varphi_{2}$ are real fields, the potential takes the form

$$
V(\phi)=V\left(\phi_{0}\right)-\mu^{2} \varphi_{1}^{2}+h v \varphi_{1}\left(\varphi_{1}^{2}+\varphi_{2}^{2}\right)+\frac{h}{4}\left(\varphi_{1}^{2}+\varphi_{2}^{2}\right)^{2} .
$$

Thus, $\varphi_{1}$ describes a massive state of mass $m_{\varphi_{1}}^{2}=-2 \mu^{2}$, while $\varphi_{2}$ is massless.

The first possibility $\left(\mu^{2}>0\right)$ is just the usual situation with a single ground state. The other case, with SSB, is more interesting. The appearance of a massless particle when $\mu^{2}<0$ is easy to understand: the field $\varphi_{2}$ describes excitations around a flat direction in the potential, i.e., into states with the same energy as the chosen ground state. Since those excitations do not cost any energy, they obviously correspond to a massless state.

The fact that there are massless excitations associated with the SSB mechanism is a completely general result, known as the Goldstone theorem [26-28]: if a Lagrangian is invariant under a continuous symmetry group $G$, but the vacuum is only invariant under a subgroup $H \subset G$, then there must exist as many massless spin-0 particles (Nambu-Goldstone bosons) as broken generators (i.e., generators of $G$ which do not belong to $H$ ).

\subsection{Massive gauge bosons}

At first sight, the Goldstone theorem has very little to do with our mass problem; in fact, it makes it worse since we want massive states and not massless ones. However, something very interesting happens when there is a local gauge symmetry [29-32].

Let us consider [3] an $S U(2)_{L}$ doublet of complex scalar fields

$$
\phi(x) \equiv\left(\begin{array}{c}
\phi^{(+)}(x) \\
\phi^{(0)}(x)
\end{array}\right) .
$$

The gauged scalar Lagrangian

$$
\begin{aligned}
\mathcal{L}_{S} & =\left(D_{\mu} \phi\right)^{\dagger} D^{\mu} \phi-\mu^{2} \phi^{\dagger} \phi-h\left(\phi^{\dagger} \phi\right)^{2} & \left(h>0, \mu^{2}<0\right), \\
D^{\mu} \phi & =\left[\partial^{\mu}+i g \widetilde{W}^{\mu}+i g^{\prime} y_{\phi} B^{\mu}\right] \phi, & y_{\phi}=Q_{\phi}-T_{3}=\frac{1}{2},
\end{aligned}
$$

is invariant under local $S U(2)_{L} \otimes U(1)_{Y}$ transformations. The value of the scalar hypercharge is fixed by the requirement of having the correct couplings between $\phi(x)$ and $A^{\mu}(x)$; i.e., the photon does not couple to $\phi^{(0)}$, and $\phi^{(+)}$has the right electric charge.

The potential is very similar to the Goldstone model one in Eq. (62). There is a infinite set of degenerate states with minimum energy, satisfying

$$
\left|\left\langle 0\left|\phi^{(0)}\right| 0\right\rangle\right|=\sqrt{\frac{-\mu^{2}}{2 h}} \equiv \frac{v}{\sqrt{2}} .
$$

Note that we have made explicit the association of the classical ground state with the quantum vacuum. Since the electric charge is a conserved quantity, only the neutral scalar field can acquire a vacuum expectation value. Once we choose a particular ground state, the $S U(2)_{L} \otimes U(1)_{Y}$ symmetry gets spontaneously broken to the electromagnetic subgroup $U(1)_{\mathrm{QED}}$, which by construction still remains a true symmetry of the vacuum. According to the Goldstone theorem three massless states should then appear.

Now, let us parametrize the scalar doublet in the general form

$$
\phi(x)=\exp \left\{i \frac{\sigma_{i}}{2} \theta^{i}(x)\right\} \frac{1}{\sqrt{2}}\left(\begin{array}{c}
0 \\
v+H(x)
\end{array}\right),
$$


with four real fields $\theta^{i}(x)$ and $H(x)$. The crucial point is that the local $S U(2)_{L}$ invariance of the Lagrangian allows us to rotate away any dependence on $\theta^{i}(x)$. These three fields are precisely the would-be massless Goldstone bosons associated with the SSB mechanism.

The covariant derivative (69) couples the scalar multiplet to the $S U(2)_{L} \otimes U(1)_{Y}$ gauge bosons. If one takes the physical (unitary) gauge $\theta^{i}(x)=0$, the kinetic piece of the scalar Lagrangian (68) takes the form:

$$
\left(D_{\mu} \phi\right)^{\dagger} D^{\mu} \phi \quad \stackrel{\theta^{i}=0}{\longrightarrow} \frac{1}{2} \partial_{\mu} H \partial^{\mu} H+(v+H)^{2}\left\{\frac{g^{2}}{4} W_{\mu}^{\dagger} W^{\mu}+\frac{g^{2}}{8 \cos ^{2} \theta_{W}} Z_{\mu} Z^{\mu}\right\} .
$$

The vacuum expectation value of the neutral scalar has generated a quadratic term for the $W^{ \pm}$and the $Z$, i.e., those gauge bosons have acquired masses:

$$
M_{Z} \cos \theta_{W}=M_{W}=\frac{1}{2} v g .
$$

Therefore, we have found a clever way of giving masses to the intermediate carriers of the weak force. We just add $\mathcal{L}_{S}$ to our $S U(2)_{L} \otimes U(1)_{Y}$ model. The total Lagrangian is invariant under gauge transformations, which guarantees the renormalizability of the associated quantum field theory [33]. However, SSB occurs. The three broken generators give rise to three massless Goldstone bosons which, owing to the underlying local gauge symmetry, can be eliminated from the Lagrangian. Going to the unitary gauge, we discover that the $W^{ \pm}$and the $Z$ (but not the $\gamma$, because $U(1)_{\text {QED }}$ is an unbroken symmetry) have acquired masses, which are moreover related as indicated in Eq. (73). Notice that Eq. (52) has now the meaning of writing the gauge fields in terms of the physical boson fields with definite mass.

It is instructive to count the number of degrees of freedom (d.o.f.). Before the SSB mechanism, the Lagrangian contains massless $W^{ \pm}$and $Z$ bosons, i.e., $3 \times 2=6$ d.o.f., due to the two possible polarizations of a massless spin-1 field, and four real scalar fields. After SSB, the three Goldstone modes are 'eaten' by the weak gauge bosons, which become massive and, therefore, acquire one additional longitudinal polarization. We have then $3 \times 3=9$ d.o.f. in the gauge sector, plus the remaining scalar particle $H$, which is called the Higgs boson. The total number of d.o.f. remains of course the same. The new longitudinal polarizations of the massive gauge bosons are nothing else than the original Goldstone fields. It was necessary to introduce additional d.o.f. (scalars) in the gauge theory in order to give masses to the gauge bosons. The Higgs appears because the scalar doublet (67) contains too many fields.

\subsection{Predictions}

We have now all the needed ingredients to describe the electroweak interaction within a well-defined quantum field theory. Our theoretical framework implies the existence of massive intermediate gauge bosons, $W^{ \pm}$and $Z$. Moreover, the chosen SSB mechanism has produced a precise prediction 1 for the $W^{ \pm}$and $Z$ masses, relating them to the vacuum expectation value of the scalar field through Eq. (73). Thus, $M_{Z}$ is predicted to be bigger than $M_{W}$ in agreement with the measured masses [34, 35]:

$$
M_{Z}=91.1875 \pm 0.0021 \mathrm{GeV}, \quad M_{W}=80.399 \pm 0.023 \mathrm{GeV} .
$$

From these experimental numbers, one obtains the electroweak mixing angle

$$
\sin ^{2} \theta_{W}=1-\frac{M_{W}^{2}}{M_{Z}^{2}}=0.223 .
$$

\footnotetext{
${ }^{1}$ Note, however, that the relation $M_{Z} \cos \theta_{W}=M_{W}$ has a more general validity. It is a direct consequence of the symmetry properties of $\mathcal{L}_{S}$ and does not depend on its detailed dynamics.
} 
We can easily get and independent estimate of $\sin ^{2} \theta_{W}$ from the decay $\mu^{-} \rightarrow e^{-} \bar{\nu}_{e} \nu_{\mu}$. The momentum transfer $q^{2}=\left(p_{\mu}-p_{\nu_{\mu}}\right)^{2}=\left(p_{e}+p_{\nu_{e}}\right)^{2} \lesssim m_{\mu}^{2}$ is much smaller than $M_{W}^{2}$. Therefore, the $W$ propagator in Fig. 6 shrinks to a point and can be well approximated through a local four-fermion interaction, i.e.,

$$
\frac{g^{2}}{M_{W}^{2}-q^{2}} \approx \frac{g^{2}}{M_{W}^{2}}=\frac{4 \pi \alpha}{\sin ^{2} \theta_{W} M_{W}^{2}} \equiv 4 \sqrt{2} G_{F} .
$$

The measured muon lifetime, $\tau_{\mu}=(2.1969803 \pm 0.0000022) \cdot 10^{-6} \mathrm{~s}$ [36], provides a very precise determination of the Fermi coupling constant $G_{F}$ :

$$
\frac{1}{\tau_{\mu}}=\Gamma_{\mu}=\frac{G_{F}^{2} m_{\mu}^{5}}{192 \pi^{3}} f\left(m_{e}^{2} / m_{\mu}^{2}\right)\left(1+\delta_{\mathrm{RC}}\right), \quad f(x) \equiv 1-8 x+8 x^{3}-x^{4}-12 x^{2} \log x .
$$

Taking into account the radiative corrections $\delta_{\mathrm{RC}}$, which are known to $O\left(\alpha^{2}\right)$ [37- -39], one gets [36]:

$$
G_{F}=(1.1663788 \pm 0.0000007) \cdot 10^{-5} \mathrm{GeV}^{-2} .
$$

The measured values of $\alpha, M_{W}$ and $G_{F}$ imply

$$
\sin ^{2} \theta_{W}=0.215,
$$

in very good agreement with Eq. (75). We shall see later that the small difference between these two numbers can be understood in terms of higher-order quantum corrections. The Fermi coupling gives also a direct determination of the electroweak scale, i.e., the scalar vacuum expectation value:

$$
v=\left(\sqrt{2} G_{F}\right)^{-1 / 2}=246 \mathrm{GeV} .
$$

\subsection{The Higgs boson}

The scalar Lagrangian in Eq. (68) has introduced a new scalar particle into the model: the Higgs $H$. In terms of the physical fields (unitary gauge), $\mathcal{L}_{S}$ takes the form

$$
\mathcal{L}_{S}=\frac{1}{4} h v^{4}+\mathcal{L}_{H}+\mathcal{L}_{H G^{2}}
$$

where

$$
\begin{gathered}
\mathcal{L}_{H}=\frac{1}{2} \partial_{\mu} H \partial^{\mu} H-\frac{1}{2} M_{H}^{2} H^{2}-\frac{M_{H}^{2}}{2 v} H^{3}-\frac{M_{H}^{2}}{8 v^{2}} H^{4} \\
\mathcal{L}_{H G^{2}}=M_{W}^{2} W_{\mu}^{\dagger} W^{\mu}\left\{1+\frac{2}{v} H+\frac{H^{2}}{v^{2}}\right\}+\frac{1}{2} M_{Z}^{2} Z_{\mu} Z^{\mu}\left\{1+\frac{2}{v} H+\frac{H^{2}}{v^{2}}\right\}
\end{gathered}
$$

and the Higgs mass is given by

$$
M_{H}=\sqrt{-2 \mu^{2}}=\sqrt{2 h} v .
$$

The Higgs interactions (Fig. 13) have a very characteristic form: they are always proportional to the mass (squared) of the coupled boson. All Higgs couplings are determined by $M_{H}, M_{W}, M_{Z}$ and the vacuum expectation value $v$.

So far the experimental searches for the Higgs have only provided negative results. The exclusion of the kinematical range accessible at LEP sets the lower bound [40]

$$
M_{H}>114.4 \mathrm{GeV} \quad(95 \% \text { C.L. }) \text {. }
$$

The most recent limits from direct searches at the LHC and the Tevatron will be discussed in Section 5. 

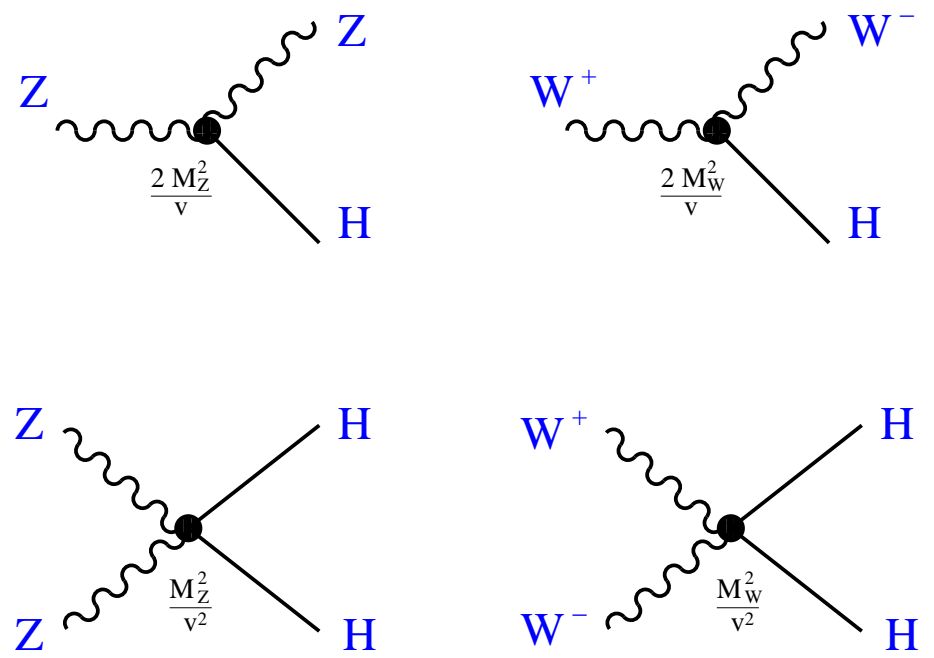

Fig. 13: Higgs couplings to the gauge bosons.

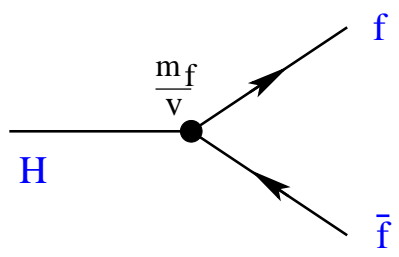

Fig. 14: Fermionic coupling of the Higgs boson.

\subsection{Fermion masses}

A fermionic mass term $\mathcal{L}_{m}=-m \bar{\psi} \psi=-m\left(\bar{\psi}_{L} \psi_{R}+\bar{\psi}_{R} \psi_{L}\right)$ is not allowed, because it breaks the gauge symmetry. However, since we have introduced an additional scalar doublet into the model, we can write the following gauge-invariant fermion-scalar coupling:

$\mathcal{L}_{Y}=-c_{1}(\bar{u}, \bar{d})_{L}\left(\begin{array}{c}\phi^{(+)} \\ \phi^{(0)}\end{array}\right) d_{R}-c_{2}(\bar{u}, \bar{d})_{L}\left(\begin{array}{c}\phi^{(0) *} \\ -\phi^{(-)}\end{array}\right) u_{R}-c_{3}\left(\bar{\nu}_{e}, \bar{e}\right)_{L}\left(\begin{array}{c}\phi^{(+)} \\ \phi^{(0)}\end{array}\right) e_{R}+$ h.c.,

where the second term involves the $\mathcal{C}$-conjugate scalar field $\phi^{c} \equiv i \sigma_{2} \phi^{*}$. In the unitary gauge, this Yukawa-type Lagrangian takes the simpler form

$$
\mathcal{L}_{Y}=-\frac{1}{\sqrt{2}}(v+H)\left\{c_{1} \bar{d} d+c_{2} \bar{u} u+c_{3} \bar{e} e\right\} .
$$

Therefore, the SSB mechanism generates also fermion masses:

$$
m_{d}=c_{1} \frac{v}{\sqrt{2}}, \quad m_{u}=c_{2} \frac{v}{\sqrt{2}}, \quad m_{e}=c_{3} \frac{v}{\sqrt{2}} .
$$

Since we do not know the parameters $c_{i}$, the values of the fermion masses are arbitrary. Note, however, that all Yukawa couplings are fixed in terms of the masses (Fig. 14):

$$
\mathcal{L}_{Y}=-\left(1+\frac{H}{v}\right)\left\{m_{d} \bar{d} d+m_{u} \bar{u} u+m_{e} \bar{e} e\right\}
$$



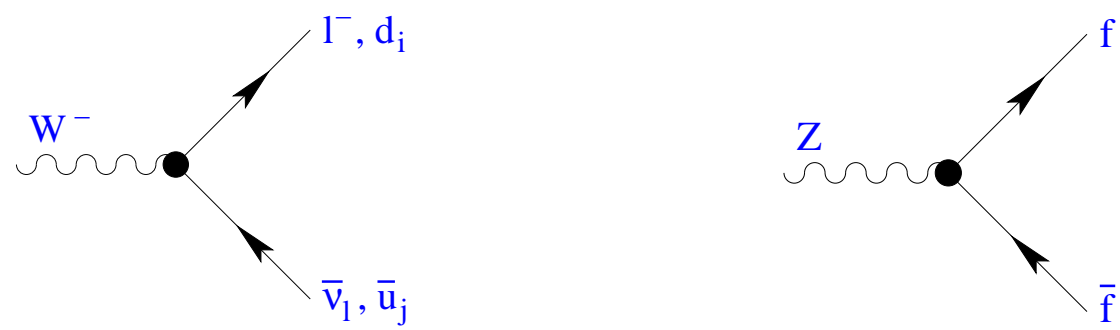

Fig. 15: Tree-level Feynman diagrams contributing to the $W^{ \pm}$and $Z$ decays.

\section{Electroweak Phenomenology}

In the gauge and scalar sectors, the SM Lagrangian contains only four parameters: $g, g^{\prime}, \mu^{2}$ and $h$. One could trade them by $\alpha, \theta_{W}, M_{W}$ and $M_{H}$. Alternatively, we can choose as free parameters:

$$
\begin{aligned}
G_{F} & =(1.1663788 \pm 0.0000007) \cdot 10^{-5} \mathrm{GeV}^{-2} \\
\alpha^{-1} & =137.035999084 \pm 0.000000051 \\
M_{Z} & =(91.1875 \pm 0.0021) \mathrm{GeV}
\end{aligned}
$$

and the Higgs mass $M_{H}$. This has the advantage of using the three most precise experimental determinations to fix the interaction. The relations

$$
\sin ^{2} \theta_{W}=1-\frac{M_{W}^{2}}{M_{Z}^{2}}, \quad M_{W}^{2} \sin ^{2} \theta_{W}=\frac{\pi \alpha}{\sqrt{2} G_{F}}
$$

determine then $\sin ^{2} \theta_{W}=0.212$ and $M_{W}=80.94 \mathrm{GeV}$. The predicted $M_{W}$ is in good agreement with the measured value in (74).

At tree level (Fig. 15), the decay widths of the weak gauge bosons can be easily computed. The $W$ partial widths,

$$
\Gamma\left(W^{-} \rightarrow \bar{\nu}_{l} l^{-}\right)=\frac{G_{F} M_{W}^{3}}{6 \pi \sqrt{2}}, \quad \quad \Gamma\left(W^{-} \rightarrow \bar{u}_{i} d_{j}\right)=N_{C}\left|\mathbf{V}_{i j}\right|^{2} \frac{G_{F} M_{W}^{3}}{6 \pi \sqrt{2}},
$$

are equal for all leptonic decay modes (up to small kinematical mass corrections). The quark modes involve also the colour quantum number $N_{C}=3$ and the mixing factor $\mathbf{V}_{i j}$ relating weak and mass eigenstates, $d_{i}^{\prime}=\mathbf{V}_{i j} d_{j}$. The $Z$ partial widths are different for each decay mode, since its couplings depend on the fermion charge:

$$
\Gamma(Z \rightarrow \bar{f} f)=N_{f} \frac{G_{F} M_{Z}^{3}}{6 \pi \sqrt{2}}\left(\left|v_{f}\right|^{2}+\left|a_{f}\right|^{2}\right),
$$

where $N_{l}=1$ and $N_{q}=N_{C}$. Summing over all possible final fermion pairs, one predicts the total widths $\Gamma_{W}=2.09 \mathrm{GeV}$ and $\Gamma_{Z}=2.48 \mathrm{GeV}$, in excellent agreement with the experimental values $\Gamma_{W}=(2.085 \pm 0.042) \mathrm{GeV}$ and $\Gamma_{Z}=(2.4952 \pm 0.0023) \mathrm{GeV}$ [34,35].

The universality of the $W$ couplings implies

$$
\operatorname{Br}\left(W^{-} \rightarrow \bar{\nu}_{l} l^{-}\right)=\frac{1}{3+2 N_{C}}=11.1 \%,
$$

where we have taken into account that the decay into the top quark is kinematically forbidden. Similarly, the leptonic decay widths of the $Z$ are predicted to be $\Gamma_{l} \equiv \Gamma\left(Z \rightarrow l^{+} l^{-}\right)=84.85 \mathrm{MeV}$. As shown in Table 2, these predictions are in good agreement with the measured leptonic widths, confirming the universality of the $W$ and $Z$ leptonic couplings. There is, however, an excess of the branching ratio $W \rightarrow \tau \bar{\nu}_{\tau}$ with respect to $W \rightarrow e \bar{\nu}_{e}$ and $W \rightarrow \mu \bar{\nu}_{\mu}$, which represents a $2.6 \sigma$ effect. 
Table 2: Measured values of $\operatorname{Br}\left(W^{-} \rightarrow \bar{\nu}_{l} l^{-}\right)$and $\Gamma\left(Z \rightarrow l^{+} l^{-}\right)$[9,34,35]. The average of the three leptonic modes is shown in the last column (for a massless charged lepton $l$ ).

\begin{tabular}{|c|c|c|c|c|}
\hline & $e$ & $\mu$ & $\tau$ & $l$ \\
\hline $\operatorname{Br}\left(W^{-} \rightarrow \bar{\nu}_{l} l^{-}\right)$ & $10.75 \pm 0.13$ & $10.57 \pm 0.15$ & $11.25 \pm 0.20$ & $10.80 \pm 0.09$ \\
\hline$\Gamma\left(Z \rightarrow l^{+} l^{-}\right) \quad(\mathrm{MeV})$ & $83.91 \pm 0.12$ & $83.99 \pm 0.18$ & $84.08 \pm 0.22$ & $83.984 \pm 0.086$ \\
\hline
\end{tabular}

Table 3: Experimental determinations of the ratios $g_{l} / g_{l^{\prime}}$ [9,41,44]

\begin{tabular}{ccccc}
\hline \hline & $\Gamma_{\tau \rightarrow \nu_{\tau} e \bar{\nu}_{e}} / \Gamma_{\mu \rightarrow \nu_{\mu} e \bar{\nu}_{e}}$ & $\Gamma_{\tau \rightarrow \nu_{\tau} \pi} / \Gamma_{\pi \rightarrow \mu} \bar{\nu}_{\mu}$ & $\Gamma_{\tau \rightarrow \nu_{\tau} K} / \Gamma_{K \rightarrow \mu \bar{\nu}_{\mu}}$ & $\Gamma_{W \rightarrow \tau \bar{\nu}_{\tau}} / \Gamma_{W \rightarrow \mu \bar{\nu}_{\mu}}$ \\
\hline$\left|g_{\tau} / g_{\mu}\right|$ & $1.0007 \pm 0.0022$ & $0.992 \pm 0.004$ & $0.982 \pm 0.008$ & $1.032 \pm 0.012$ \\
\hline \hline & $\Gamma_{\tau \rightarrow \nu_{\tau} \mu \bar{\nu}_{\mu}} / \Gamma_{\tau \rightarrow \nu_{\tau} e \bar{\nu}_{e}}$ & $\Gamma_{\pi \rightarrow \mu \bar{\nu}_{\mu}} / \Gamma_{\pi \rightarrow e \bar{\nu}_{e}}$ & $\Gamma_{K \rightarrow \mu \bar{\nu}_{\mu}} / \Gamma_{K \rightarrow e \bar{\nu}_{e}}$ & $\Gamma_{K \rightarrow \pi \mu \bar{\nu}_{\mu}} / \Gamma_{K \rightarrow \pi e \bar{\nu}_{e}}$ \\
\hline$\left|g_{\mu} / g_{e}\right|$ & $1.0018 \pm 0.0014$ & $1.0021 \pm 0.0016$ & $0.998 \pm 0.002$ & $1.001 \pm 0.002$ \\
\hline \hline & $\Gamma_{W \rightarrow \mu \bar{\nu}_{\mu}} / \Gamma_{W \rightarrow e \bar{\nu}_{e}}$ & & $\Gamma_{\tau \rightarrow \nu_{\tau} \mu \bar{\nu}_{\mu}} / \Gamma_{\mu \rightarrow \nu_{\mu} e \bar{\nu}_{e}}$ & $\Gamma_{W \rightarrow \tau \bar{\nu}_{\tau}} / \Gamma_{W \rightarrow e \bar{\nu}_{e}}$ \\
\hline$\left|g_{\mu} / g_{e}\right|$ & $0.991 \pm 0.009$ & $\left|g_{\tau} / g_{e}\right|$ & $1.0016 \pm 0.0021$ & $1.023 \pm 0.011$ \\
\hline \hline
\end{tabular}

The universality of the leptonic $W$ couplings can also be tested indirectly, through weak decays mediated by charged-current interactions. Comparing the measured decay widths of leptonic or semileptonic decays which only differ by the lepton flavour, one can test experimentally that the $W$ interaction is indeed the same, i.e., that $g_{e}=g_{\mu}=g_{\tau} \equiv g$. As shown in Table 3, the present data verify the universality of the leptonic charged-current couplings to the $0.2 \%$ level.

Another interesting quantity is the $Z$ decay width into invisible modes,

$$
\frac{\Gamma_{\text {inv }}}{\Gamma_{l}} \equiv \frac{N_{\nu} \Gamma(Z \rightarrow \bar{\nu} \nu)}{\Gamma_{l}}=\frac{2 N_{\nu}}{\left(1-4 \sin ^{2} \theta_{W}\right)^{2}+1},
$$

which is usually normalized to the charged leptonic width. The comparison with the measured value, $\Gamma_{\text {inv }} / \Gamma_{l}=5.943 \pm 0.016$ [34,35], provides very strong experimental evidence for the existence of three different light neutrinos.

\subsection{Fermion-pair production at the $Z$ peak}

Additional information can be obtained from the study of the process $e^{+} e^{-} \rightarrow \gamma, Z \rightarrow \bar{f} f$ (Fig. 16). For unpolarized $e^{+}$and $e^{-}$beams, the differential cross-section can be written, at lowest order, as

$$
\frac{d \sigma}{d \Omega}=\frac{\alpha^{2}}{8 s} N_{f}\left\{A\left(1+\cos ^{2} \theta\right)+B \cos \theta-h_{f}\left[C\left(1+\cos ^{2} \theta\right)+D \cos \theta\right]\right\}
$$

where $h_{f}= \pm 1$ denotes the sign of the helicity of the produced fermion $f$, and $\theta$ is the scattering angle between $e^{-}$and $f$ in the centre-of-mass system. Here,

$$
\begin{aligned}
& A=1+2 v_{e} v_{f} \operatorname{Re}(\chi)+\left(v_{e}^{2}+a_{e}^{2}\right)\left(v_{f}^{2}+a_{f}^{2}\right)|\chi|^{2}, \\
& B=4 a_{e} a_{f} \operatorname{Re}(\chi)+8 v_{e} a_{e} v_{f} a_{f}|\chi|^{2}, \\
& C=2 v_{e} a_{f} \operatorname{Re}(\chi)+2\left(v_{e}^{2}+a_{e}^{2}\right) v_{f} a_{f}|\chi|^{2}, \\
& D=4 a_{e} v_{f} \operatorname{Re}(\chi)+4 v_{e} a_{e}\left(v_{f}^{2}+a_{f}^{2}\right)|\chi|^{2},
\end{aligned}
$$



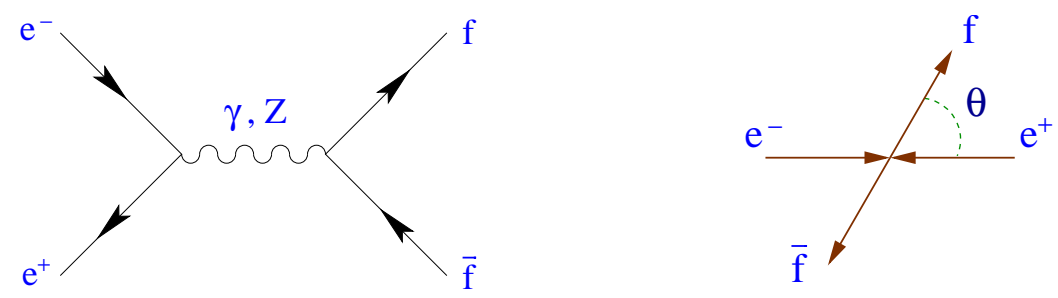

Fig. 16: Tree-level contributions to $e^{+} e^{-} \rightarrow \bar{f} f$ and kinematical configuration in the centre-of-mass system.

and $\chi$ contains the $Z$ propagator

$$
\chi=\frac{G_{F} M_{Z}^{2}}{2 \sqrt{2} \pi \alpha} \frac{s}{s-M_{Z}^{2}+i s \Gamma_{Z} / M_{Z}} .
$$

The coefficients $A, B, C$ and $D$ can be experimentally determined by measuring the total crosssection, the forward-backward asymmetry, the polarization asymmetry, and the forward-backward polarization asymmetry, respectively:

$$
\begin{gathered}
\sigma(s)=\frac{4 \pi \alpha^{2}}{3 s} N_{f} A, \\
\mathcal{A}_{\mathrm{FB}}(s) \equiv \frac{N_{F}-N_{B}}{N_{F}+N_{B}}=\frac{3}{8} \frac{B}{A}, \\
\mathcal{A}_{\mathrm{Pol}}(s) \equiv \frac{\sigma^{\left(h_{f}=+1\right)}-\sigma^{\left(h_{f}=-1\right)}}{\sigma^{\left(h_{f}=+1\right)}+\sigma^{\left(h_{f}=-1\right)}}=-\frac{C}{A}, \\
\mathcal{A}_{\mathrm{FB}, \mathrm{Pol}}(s) \equiv \frac{N_{F}^{\left(h_{f}=+1\right)}-N_{F}^{\left(h_{f}=-1\right)}-N_{B}^{\left(h_{f}=+1\right)}+N_{B}^{\left(h_{f}=-1\right)}}{N_{F}^{\left(h_{f}=+1\right)}+N_{F}^{\left(h_{f}=-1\right)}+N_{B}^{\left(h_{f}=+1\right)}+N_{B}^{\left(h_{f}=-1\right)}}=-\frac{3}{8} \frac{D}{A} .
\end{gathered}
$$

Here, $N_{F}$ and $N_{B}$ denote the number of $f$ 's emerging in the forward and backward hemispheres, respectively, with respect to the electron direction. The measurement of the final fermion polarization can be done for $f=\tau$ by measuring the distribution of the final $\tau$ decay products.

For $s=M_{Z}^{2}$, the real part of the $Z$ propagator vanishes and the photon-exchange terms can be neglected in comparison with the $Z$-exchange contributions $\left(\Gamma_{Z}^{2} / M_{Z}^{2}<<1\right)$. Equations (99) become then,

$$
\begin{array}{rlrl}
\sigma^{0, f} & \equiv \sigma\left(M_{Z}^{2}\right)=\frac{12 \pi}{M_{Z}^{2}} \frac{\Gamma_{e} \Gamma_{f}}{\Gamma_{Z}^{2}}, & \mathcal{A}_{\mathrm{FB}}^{0, f} & \equiv \mathcal{A}_{F B}\left(M_{Z}^{2}\right)=\frac{3}{4} \mathcal{P}_{e} \mathcal{P}_{f}, \\
\mathcal{A}_{\mathrm{Pol}}^{0, f} \equiv \mathcal{A}_{\mathrm{Pol}}\left(M_{Z}^{2}\right)=\mathcal{P}_{f}, & \mathcal{A}_{\mathrm{FB}, \mathrm{Pol}}^{0, f} \equiv \mathcal{A}_{\mathrm{FB}, \mathrm{Pol}}\left(M_{Z}^{2}\right)=\frac{3}{4} \mathcal{P}_{e}
\end{array}
$$

where $\Gamma_{f}$ is the $Z$ partial decay width into the $\bar{f} f$ final state, and

$$
\mathcal{P}_{f} \equiv-A_{f} \equiv \frac{-2 v_{f} a_{f}}{v_{f}^{2}+a_{f}^{2}}
$$

is the average longitudinal polarization of the fermion $f$, which only depends on the ratio of the vector and axial-vector couplings.

With polarized $e^{+} e^{-}$beams, which have been available at SLC, one can also study the left-right asymmetry between the cross-sections for initial left- and right-handed electrons, and the corresponding forward-backward left-right asymmetry:

$$
\mathcal{A}_{\mathrm{LR}}^{0} \equiv \mathcal{A}_{\mathrm{LR}}\left(M_{Z}^{2}\right)=\frac{\sigma_{L}\left(M_{Z}^{2}\right)-\sigma_{R}\left(M_{Z}^{2}\right)}{\sigma_{L}\left(M_{Z}^{2}\right)+\sigma_{R}\left(M_{Z}^{2}\right)}=-\mathcal{P}_{e}, \quad \mathcal{A}_{\mathrm{FB}, \mathrm{LR}}^{0, f} \equiv \mathcal{A}_{\mathrm{FB}, \mathrm{LR}}\left(M_{Z}^{2}\right)=-\frac{3}{4} \mathcal{P}_{f}
$$


At the $Z$ peak, $\mathcal{A}_{\mathrm{LR}}^{0}$ measures the average initial lepton polarization, $\mathcal{P}_{e}$, without any need for final particle identification, while $\mathcal{A}_{\mathrm{FB}, \mathrm{LR}}^{0, f}$ provides a direct determination of the final fermion polarization.

$\mathcal{P}_{f}$ is a very sensitive function of $\sin ^{2} \theta_{W}$. Small higher-order corrections can produce large variations on the predicted lepton polarization because $\left|v_{l}\right|=\frac{1}{2}\left|1-4 \sin ^{2} \theta_{W}\right| \ll 1$. Therefore, $\mathcal{P}_{l}$ provides an interesting window to search for electroweak quantum effects.

\subsection{QED and QCD corrections}
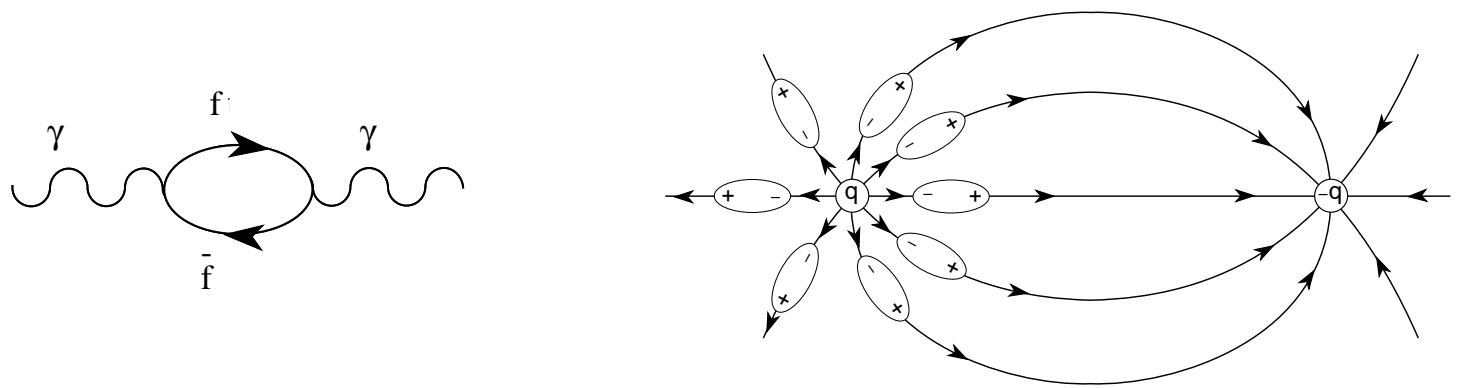

Fig. 17: The photon vacuum polarization (left) generates a charge screening effect, making $\alpha(s)$ smaller at larger distances.

Before trying to analyse the relevance of higher-order electroweak contributions, it is instructive to consider the numerical impact of the well-known QED and QCD corrections. The photon propagator gets vacuum polarization corrections, induced by virtual fermion-antifermion pairs. This kind of QED loop corrections can be taken into account through a redefinition of the QED coupling, which depends on the energy scale. The resulting QED running coupling $\alpha(s)$ decreases at large distances. This can be intuitively understood as the charge screening generated by the virtual fermion pairs (Fig. 17). The physical QED vacuum behaves as a polarized dielectric medium. The huge difference between the electron and $Z$ mass scales makes this quantum correction relevant at LEP energies [15, 34, 35]:

$$
\alpha\left(m_{e}^{2}\right)^{-1}=137.035999084 \pm 0.000000051>\alpha\left(M_{Z}^{2}\right)^{-1}=128.95 \pm 0.05 .
$$

The running effect generates an important change in Eq. (91). Since $G_{F}$ is measured at low energies, while $M_{W}$ is a high-energy parameter, the relation between both quantities is modified by vacuum-polarization contributions. Changing $\alpha$ by $\alpha\left(M_{Z}^{2}\right)$, one gets the corrected predictions:

$$
\sin ^{2} \theta_{W}=0.231, \quad M_{W}=79.96 \mathrm{GeV} .
$$

The experimental value of $M_{W}$ is in the range between the two results obtained with either $\alpha$ or $\alpha\left(M_{Z}^{2}\right)$, showing its sensitivity to quantum corrections. The effect is more spectacular in the leptonic asymmetries at the $Z$ peak. The small variation of $\sin ^{2} \theta_{W}$ from 0.212 to 0.231 induces a large shift on the vector $Z$ coupling to charged leptons from $v_{l}=-0.076$ to -0.038 , changing the predicted average lepton polarization $\mathcal{P}_{l}$ by a factor of two.

So far, we have treated quarks and leptons on an equal footing. However, quarks are stronginteracting particles. The gluonic corrections to the decays $Z \rightarrow \bar{q} q$ and $W^{-} \rightarrow \bar{u}_{i} d_{j}$ can be directly incorporated into the formulae given before by taking an 'effective' number of colours:

$$
N_{C} \quad \Longrightarrow \quad N_{C}\left\{1+\frac{\alpha_{s}}{\pi}+\ldots\right\} \approx 3.115,
$$

where we have used the value of $\alpha_{s}$ at $s=M_{Z}^{2}, \alpha_{s}\left(M_{Z}^{2}\right)=0.1183 \pm 0.0010$ [45, 46].

Note that the strong coupling also 'runs'. However, the gluon self-interactions generate an antiscreening effect, through gluon-loop corrections to the gluon propagator which spread out the QCD 
charge [6]. Since this correction is larger than the screening of the colour charge induced by virtual quark-antiquark pairs, the net result is that the strong coupling decreases at short distances. Thus, QCD has the required property of asymptotic freedom: quarks behave as free particles when $Q^{2} \rightarrow \infty$ [47, 48].

QCD corrections increase the probabilities of the $Z$ and the $W^{ \pm}$to decay into hadronic modes. Therefore, their leptonic branching fractions become smaller. The effect can be easily estimated from Eq. (94). The probability of the decay $W^{-} \rightarrow \bar{\nu}_{e} e^{-}$gets reduced from $11.1 \%$ to $10.8 \%$, improving the agreement with the measured value in Table 2

\subsection{Higher-order electroweak corrections}
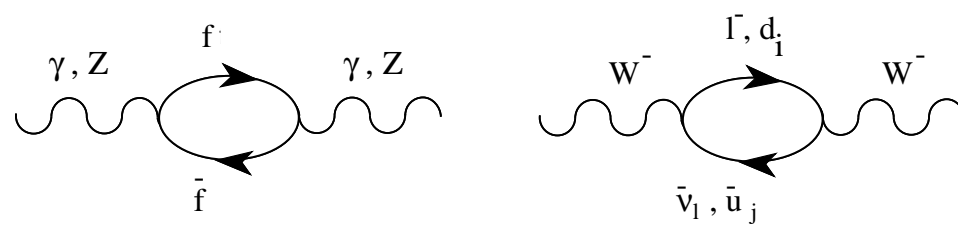

Fig. 18: Self-energy corrections to the gauge boson propagators.

Quantum corrections offer the possibility to be sensitive to heavy particles, which cannot be kinematically accessed, through their virtual loop effects. In QED and QCD the vacuum polarization contribution of a heavy fermion pair is suppressed by inverse powers of the fermion mass. At low energies, the information on the heavy fermions is then lost. This 'decoupling' of the heavy fields happens in theories with only vector couplings and an exact gauge symmetry [49], where the effects generated by the heavy particles can always be reabsorbed into a redefinition of the low-energy parameters.

The SM involves, however, a broken chiral gauge symmetry. This has the very interesting implication of avoiding the decoupling theorem [49]. The vacuum polarization contributions induced by a heavy top generate corrections to the $W^{ \pm}$and $Z$ propagators (Fig. 18), which increase quadratically with the top mass [50]. Therefore, a heavy top does not decouple. For instance, with $m_{t}=173 \mathrm{GeV}$, the leading quadratic correction to the second relation in Eq. (91) amounts to a sizeable $3 \%$ effect. The quadratic mass contribution originates in the strong breaking of weak isospin generated by the top and bottom quark masses, i.e., the effect is actually proportional to $m_{t}^{2}-m_{b}^{2}$.

Owing to an accidental $S U(2)_{C}$ symmetry of the scalar sector (the so-called custodial symmetry), the virtual production of Higgs particles does not generate any quadratic dependence on the Higgs mass at one loop [50]. The dependence on $M_{H}$ is only logarithmic. The numerical size of the corresponding correction in Eq. (91) varies from a $0.1 \%$ to a $1 \%$ effect for $M_{H}$ in the range from 100 to $1000 \mathrm{GeV}$.

Higher-order corrections to the different electroweak couplings are non-universal and usually smaller than the self-energy contributions. There is one interesting exception, the $Z \bar{b} b$ vertex (Fig. 19], which is sensitive to the top quark mass [51]. The $Z \bar{f} f$ vertex gets one-loop corrections where a virtual $W^{ \pm}$is exchanged between the two fermionic legs. Since the $W^{ \pm}$coupling changes the fermion flavour, the decays $Z \rightarrow \bar{d} d, \bar{s} s, \bar{b} b$ get contributions with a top quark in the internal fermionic lines, i.e., $Z \rightarrow \bar{t} t \rightarrow \bar{d}_{i} d_{i}$. Notice that this mechanism can also induce the flavour-changing neutral-current decays $Z \rightarrow \bar{d}_{i} d_{j}$ with $i \neq j$. These amplitudes are suppressed by the small CKM mixing factors $\left|\mathbf{V}_{t j} \mathbf{V}_{t i}^{*}\right|^{2}$. However, for the $Z \rightarrow \bar{b} b$ vertex, there is no suppression because $\left|\mathbf{V}_{t b}\right| \approx 1$.

The explicit calculation [51-54] shows the presence of hard $m_{t}^{2}$ corrections to the $Z \rightarrow \bar{b} b$ vertex. This effect can be easily understood [51] in non-unitary gauges where the unphysical charged scalar $\phi^{( \pm)}$is present. The fermionic couplings of the charged scalar are proportional to the fermion masses; therefore the exchange of a virtual $\phi^{( \pm)}$gives rise to a $m_{t}^{2}$ factor. In the unitary gauge, the charged scalar has been 'eaten' by the $W^{ \pm}$field; thus the effect comes now from the exchange of a longitudinal $W^{ \pm}$, with terms proportional to $q^{\mu} q^{\nu}$ in the propagator that generate fermion masses. Since the $W^{ \pm}$ 

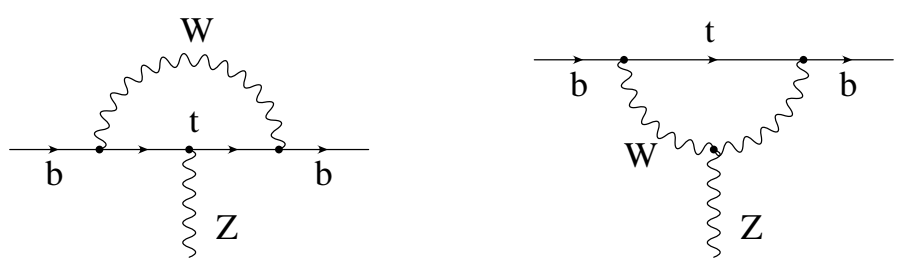

Fig. 19: One-loop corrections to the $Z \bar{b} b$ vertex, involving a virtual top.

couples only to left-handed fermions, the induced correction is the same for the vector and axial-vector $Z \bar{b} b$ couplings and, for $m_{t}=173 \mathrm{GeV}$, amounts to a $1.6 \%$ reduction of the $Z \rightarrow \bar{b} b$ decay width [51].

The 'non-decoupling' present in the $Z \bar{b} b$ vertex is quite different from the one happening in the boson self-energies. The vertex correction is not dependent on the Higgs mass. Moreover, while any kind of new heavy particle coupling to the gauge bosons would contribute to the $W$ and $Z$ self-energies, the possible new physics contributions to the $Z \bar{b} b$ vertex are much more restricted and, in any case, different. Therefore, the independent experimental measurement of the two effects is very valuable in order to disentangle possible new-physics contributions from the SM corrections. In addition, since the 'non-decoupling' vertex effect is related to $W_{L}$ exchange, it is sensitive to the SSB mechanism.

\subsection{SM electroweak fit}
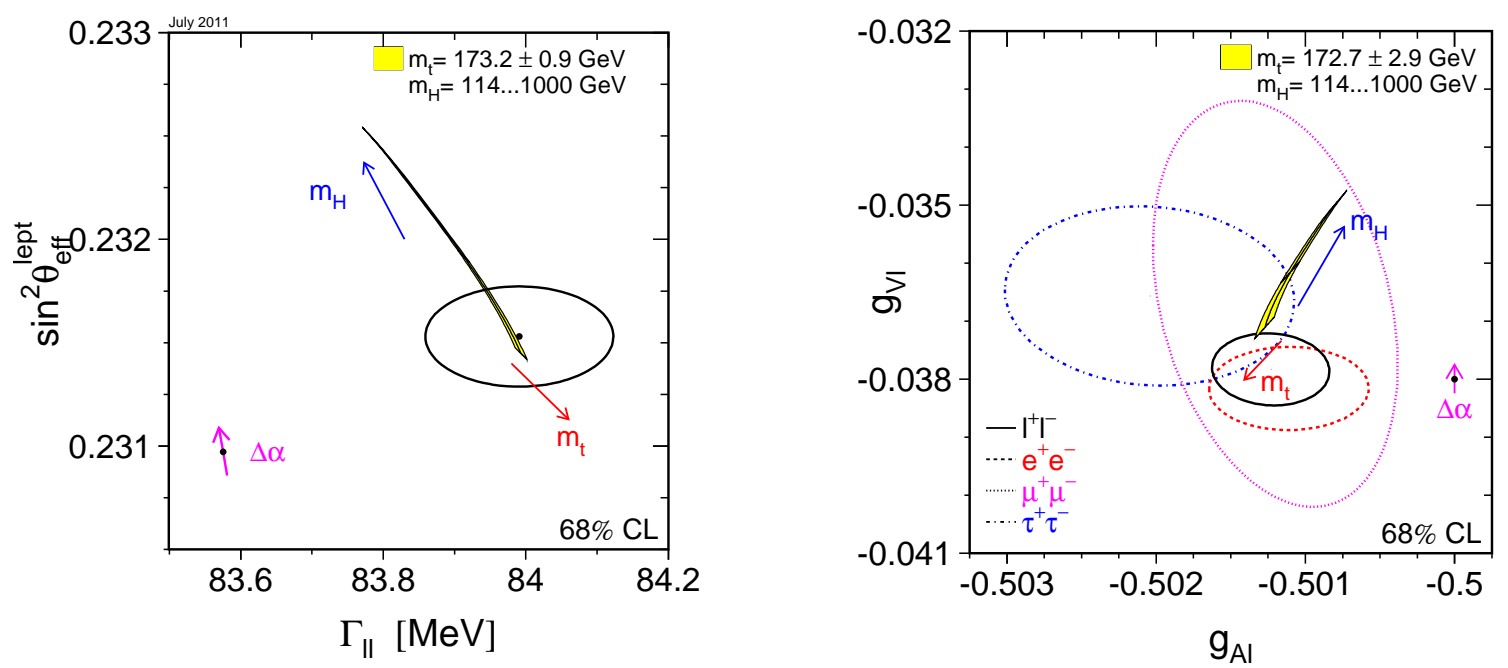

Fig. 20: Combined LEP and SLD measurements of $\sin ^{2} \theta_{\text {eff }}^{\text {lept }}$ and $\Gamma_{l}$ (left) and the corresponding effective vector and axial-vector couplings $v_{l}$ and $a_{l}$ (right). The shaded region shows the SM prediction. The arrows point in the direction of increasing values of $m_{t}$ and $M_{H}$. The point shows the predicted values if, among the electroweak radiative corrections, only the photon vacuum polarization is included. Its arrow indicates the variation induced by the uncertainty in $\alpha\left(M_{Z}^{2}\right)$ [34,35].

The leptonic asymmetry measurements from LEP and SLD can all be combined to determine the ratios $v_{l} / a_{l}$ of the vector and axial-vector couplings of the three charged leptons, or equivalently the effective electroweak mixing angle

$$
\sin ^{2} \theta_{\mathrm{eff}}^{\text {lept }} \equiv \frac{1}{4}\left(1-\frac{v_{l}}{a_{l}}\right) .
$$




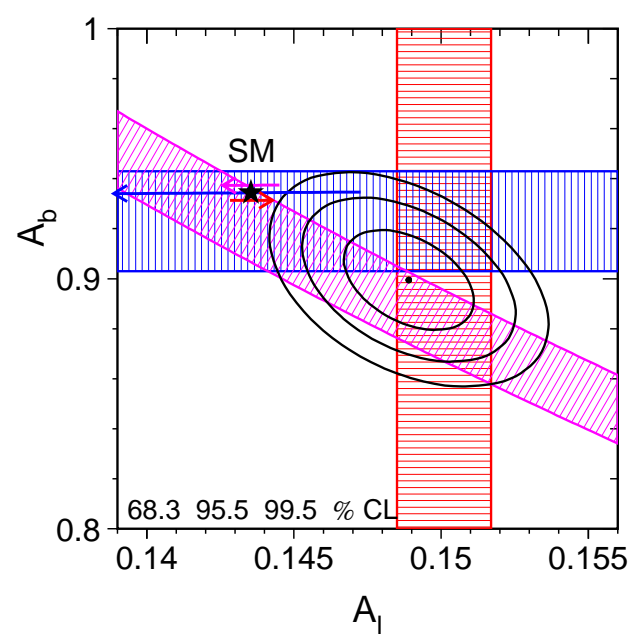

Fig. 21: Measurements of $A_{l}, A_{b}$ (SLD) and $\mathcal{A}_{\mathrm{FB}}^{0, b}$. The arrows pointing to the left (right) show the variations of the SM prediction with $M_{H}=$ $300{ }_{-186}^{+700} \mathrm{GeV}\left(m_{t}=172.7 \pm 2.9 \mathrm{GeV}\right)$. The small arrow oriented to the left shows the additional uncertainty from $\alpha\left(M_{Z}^{2}\right)[34,35]$.

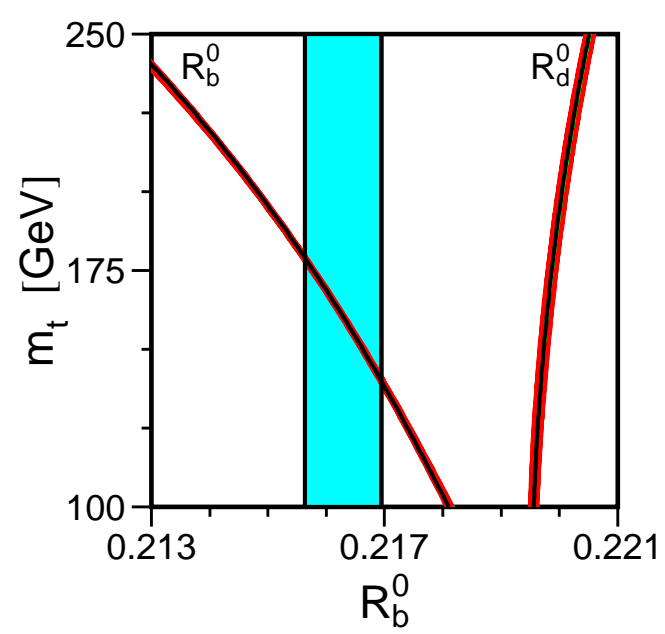

Fig. 22: The SM prediction of the ratios $R_{b}$ and $R_{d}$ $\left[R_{q} \equiv \Gamma(Z \rightarrow \bar{q} q) / \Gamma(Z \rightarrow\right.$ hadrons $\left.)\right]$, as a function of the top mass. The measured value of $R_{b}$ (vertical band) provides a determination of $m_{t}[34,35]$.

The sum $\left(v_{l}^{2}+a_{l}^{2}\right)$ is derived from the leptonic decay widths of the $Z$, i.e., from Eq. (93) corrected with a multiplicative factor $\left(1+\frac{3}{4} \frac{\alpha}{\pi}\right)$ to account for final-state QED corrections. The signs of $v_{l}$ and $a_{l}$ are fixed by requiring $a_{e}<0$.

The resulting 68\% probability contours are shown in Fig. 20, which provides strong evidence of the electroweak radiative corrections. The good agreement with the SM predictions, obtained for low values of the Higgs mass, is lost if only the QED vacuum polarization contribution is taken into account, as indicated by the point with an arrow. Notice that the uncertainty induced by the input value of $\alpha\left(M_{Z}^{2}\right)^{-1}=128.95 \pm 0.05$ is sizeable. The measured couplings of the three charged leptons confirm lepton universality in the neutral-current sector. The solid contour combines the three measurements assuming universality.

The neutrino couplings can also be determined from the invisible $Z$ decay width, by assuming three identical neutrino generations with left-handed couplings, and fixing the sign from neutrino scattering data. Alternatively, one can use the SM prediction for $\Gamma_{\text {inv }}$ to get a determination of the number of light neutrino flavours [34,35]:

$$
N_{\nu}=2.9840 \pm 0.0082
$$

Figure 21 shows the measured values of $A_{l}$ and $A_{b}$, together with the joint constraint obtained from $\mathcal{A}_{\mathrm{FB}}^{0, b}$ (diagonal band). The direct measurement of $A_{b}$ at SLD agrees well with the SM prediction; however, a much lower value is obtained from the ratio $\frac{4}{3} \mathcal{A}_{\mathrm{FB}}^{0, b} / A_{l}$. This is the most significant discrepancy observed in the $Z$-pole data. Heavy quarks $\left(\frac{4}{3} \mathcal{A}_{\mathrm{FB}}^{0, b} / A_{b}\right)$ seem to prefer a high value of the Higgs mass, while leptons $\left(A_{l}\right)$ favour a light Higgs. The combined analysis prefers low values of $M_{H}$, because of the influence of $A_{l}$.

The strong sensitivity of the ratio $R_{b} \equiv \Gamma(Z \rightarrow \bar{b} b) / \Gamma(Z \rightarrow$ hadrons) to the top quark mass is shown in Fig. 22, Owing to the $\left|V_{t d}\right|^{2}$ suppression, such a dependence is not present in the analogous ratio $R_{d}$. Combined with all other electroweak precision measurements at the $Z$ peak, $R_{b}$ provides a determination of $m_{t}$ in good agreement with the direct and most precise measurement at the Tevatron, $m_{t}=(173.2 \pm 0.9) \mathrm{GeV}$ [25]. This is shown in Fig. 23, which compares the information on $M_{W}$ and $m_{t}$ obtained at LEP1 and SLD, with the direct measurements performed at LEP2 and the Tevatron. A 

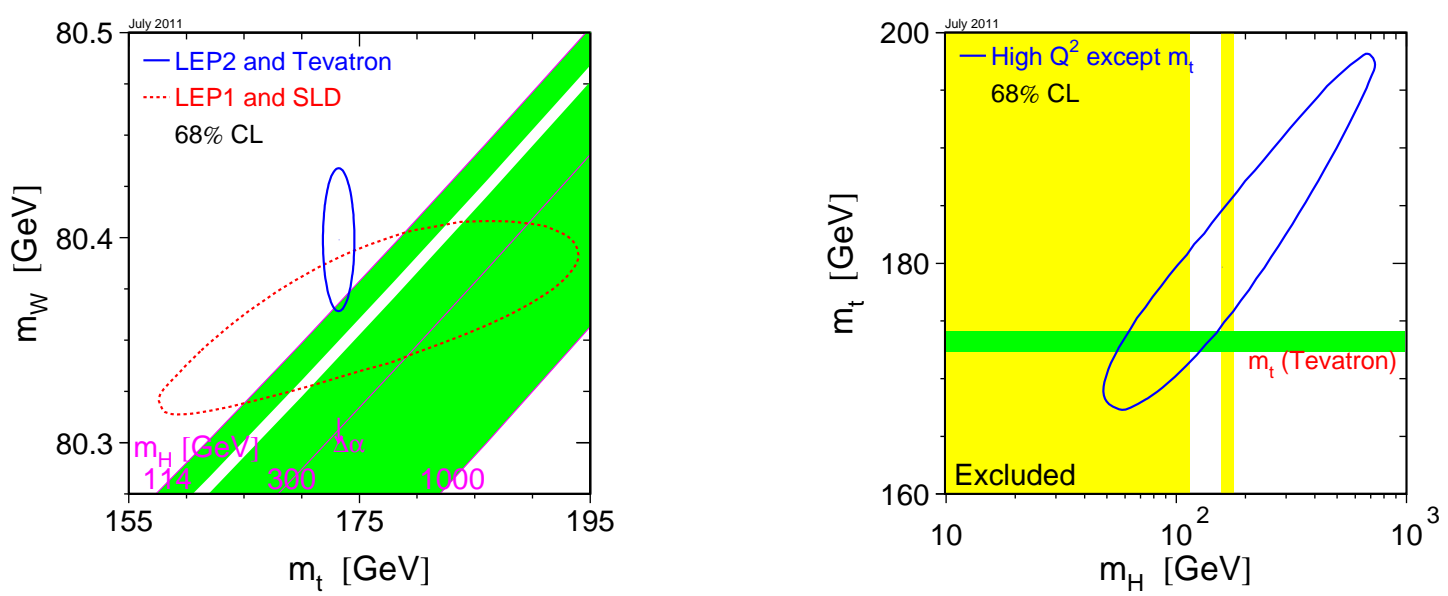

Fig. 23: Comparison (left) of the direct measurements of $M_{W}$ and $m_{t}$ (LEP2 and Tevatron data) with the indirect determination through electroweak radiative corrections (LEP1 and SLD). Also shown in the SM relationship for the masses as function of $M_{H}$. The figure on the right makes the analogous comparison for $m_{t}$ and $M_{H}$ [34,35].

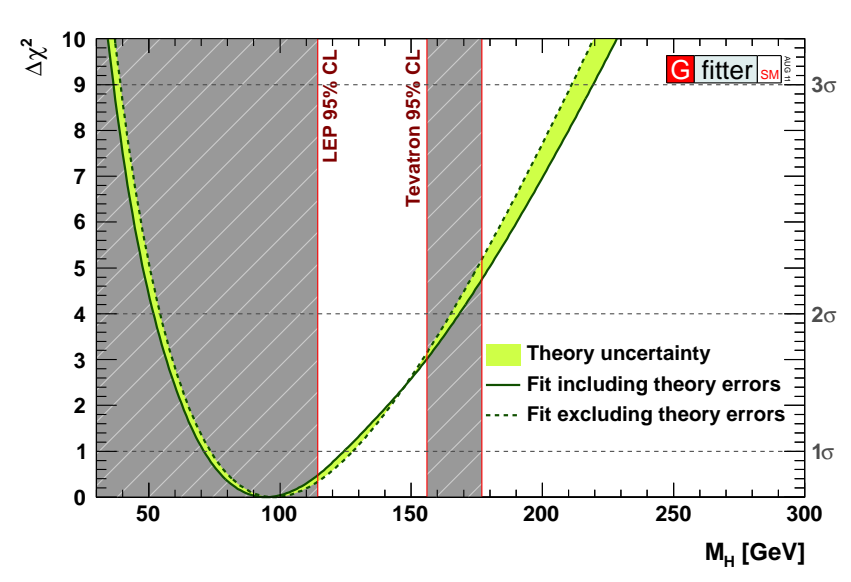

Fig. 24: $\Delta \chi^{2}$ versus $M_{H}$, from the global fit to the electroweak data. The vertical bands indicate the $95 \%$ exclusion limits from direct searches [55].

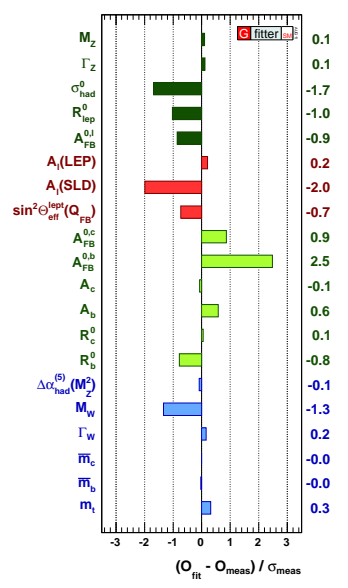

Fig. 25: Comparison between the measurements included in the combined analysis of the SM and the results from the global electroweak fit [55].

similar comparison for $m_{t}$ and $M_{H}$ is also shown.

Taking all direct and indirect data into account, one obtains the best constraints on $M_{H}$. The global electroweak fit results in the $\Delta \chi^{2}=\chi^{2}-\chi_{\min }^{2}$ curve shown in Fig. 24 The lower limit on $M_{H}$ obtained from direct searches at LEP [Eq. [85] ] is close to the point of minimum $\chi^{2}$, excluding a large portion of the allowed domain from precision measurements. The electroweak fit puts the upper bound [55]

$$
M_{H}<169 \mathrm{GeV} \quad \text { (95\% C.L.). }
$$

The fit provides also a very accurate value of the strong coupling constant, $\alpha_{s}\left(M_{Z}^{2}\right)=0.1193 \pm 0.0028$, in very good agreement with the world average value $\alpha_{s}\left(M_{Z}^{2}\right)=0.1183 \pm 0.0010$ [45, 46]. The largest discrepancy between theory and experiment occurs for $\mathcal{A}_{\mathrm{FB}}^{0, b}$, with the fitted value being $2.5 \sigma$ larger than the measurement. As shown in Fig. 25, a good agreement is obtained for all other observables. 


\subsection{Gauge self-interactions}
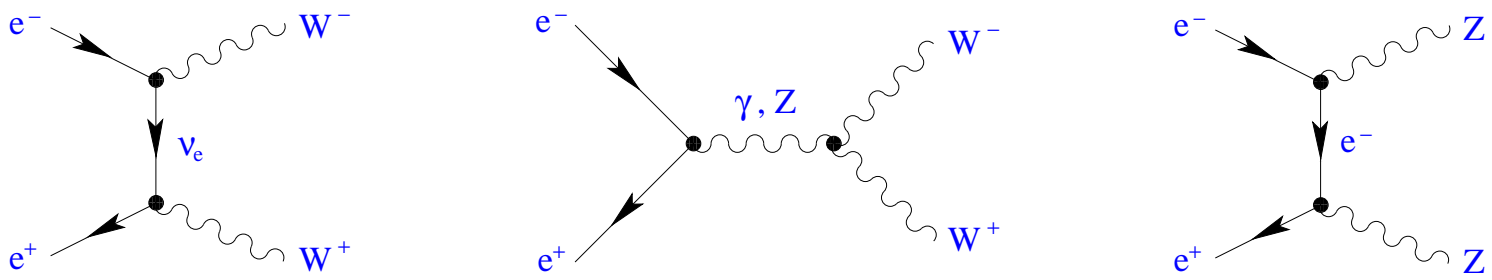

Fig. 26: Feynman diagrams contributing to $e^{+} e^{-} \rightarrow W^{+} W^{-}$and $e^{+} e^{-} \rightarrow Z Z$.

At tree level, the $W$-pair production process $e^{+} e^{-} \rightarrow W^{+} W^{-}$involves three different contributions (Fig.26), corresponding to the exchange of $\nu_{e}, \gamma$ and $Z$. The cross-section measured at LEP2 agrees very well with the SM predictions. As shown in Fig. 27, the $\nu_{e}$-exchange contribution alone would lead to an unphysical growing of the cross-section at large energies and, therefore, would imply a violation of unitarity. Adding the $\gamma$-exchange contribution softens this behaviour, but a clear disagreement with the data persists. The $Z$-exchange mechanism, which involves the $Z W W$ vertex, appears to be crucial in order to explain the data.

Since the $Z$ is electrically neutral, it does not interact with the photon. Moreover, the SM does not include any local $Z Z Z$ vertex. Therefore, the $e^{+} e^{-} \rightarrow Z Z$ cross-section only involves the contribution from $e$ exchange. The agreement of the SM predictions with the experimental measurements in both production channels, $W^{+} W^{-}$and $Z Z$, provides a test of the gauge self-interactions. There is a clear signal of the presence of a $Z W W$ vertex, with the predicted strength, and no evidence for any $\gamma Z Z$ or $Z Z Z$ interactions. The gauge structure of the $S U(2)_{L} \otimes U(1)_{Y}$ theory is nicely confirmed by the data.
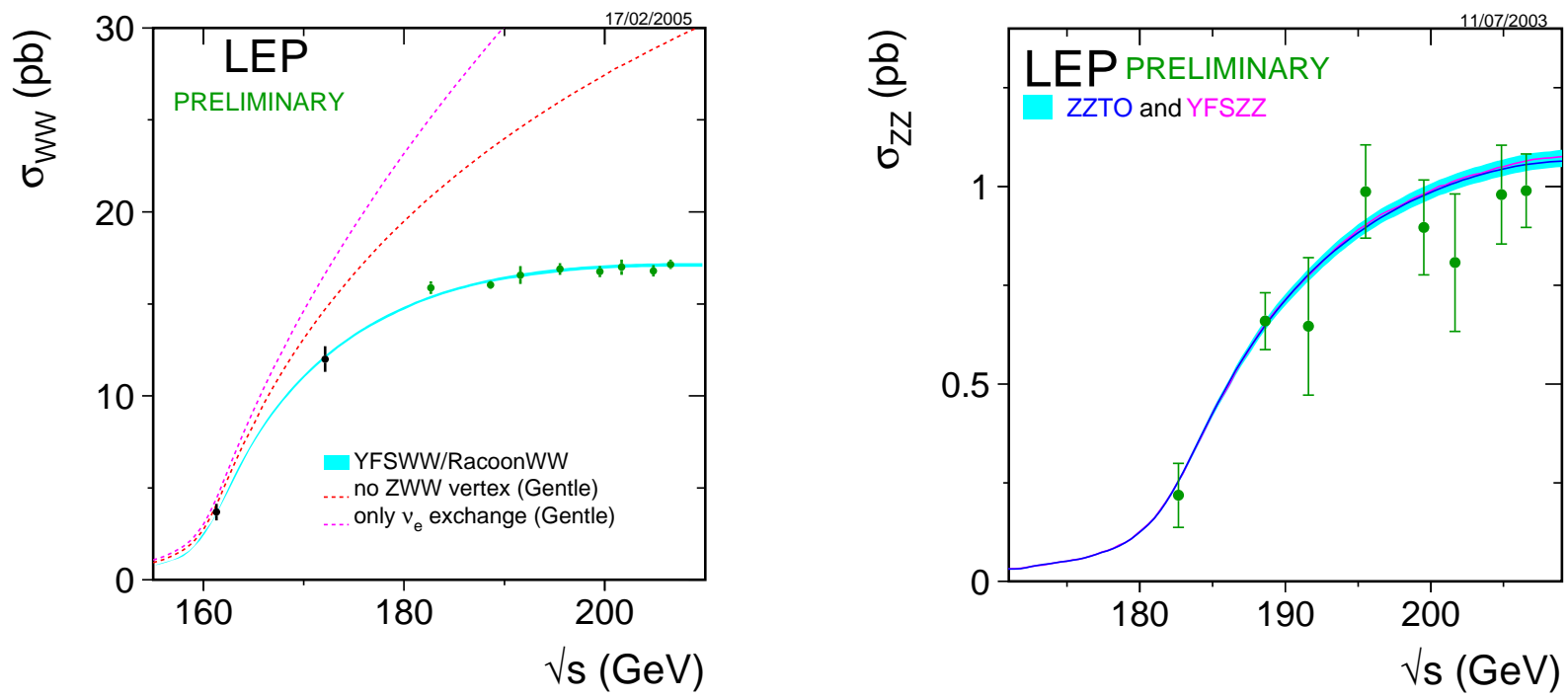

Fig. 27: Measured energy dependence of $\sigma\left(e^{+} e^{-} \rightarrow W^{+} W^{-}\right)$(left) and $\sigma\left(e^{+} e^{-} \rightarrow Z Z\right)$ (right). The three curves shown for the $W$-pair production cross-section correspond to only the $\nu_{e}$-exchange contribution (upper curve), $\nu_{e}$ exchange plus photon exchange (middle curve) and all contributions including also the $Z W W$ vertex (lower curve). Only the $e$-exchange mechanism contributes to $Z$-pair production [34,35].

\subsection{Higgs searches}

The couplings of the Higgs boson are always proportional to some mass scale. The $H f \bar{f}$ interaction grows linearly with the fermion mass, while the $H W W$ and $H Z Z$ vertices are proportional to $M_{W}^{2}$ and $M_{Z}^{2}$, respectively. Therefore, the most probable decay mode of the Higgs is the one into the heaviest possible final state. This is clearly illustrated in Fig. 28 , The $H \rightarrow b \bar{b}$ decay channel is by far the dominant 

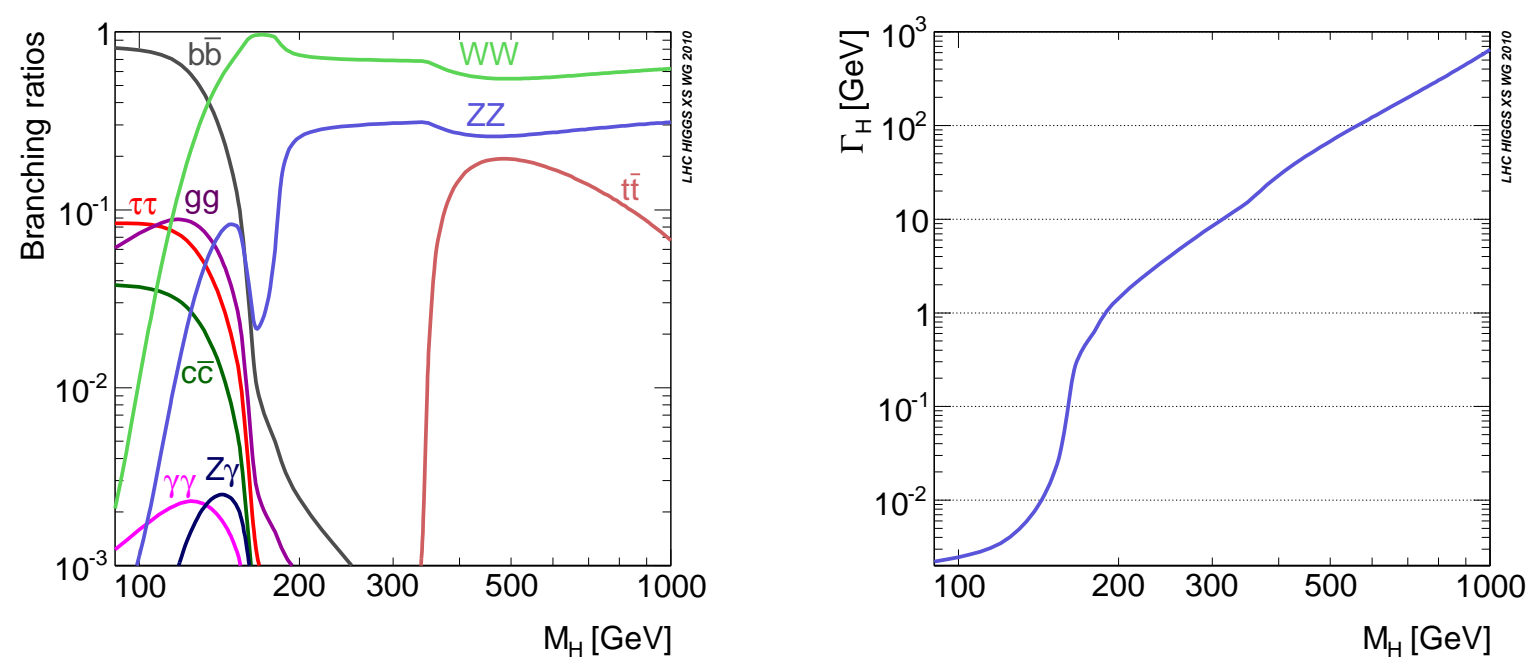

Fig. 28: Branching fractions of the different Higgs decay modes (left) and total decay width of the Higgs boson (right) as function of $M_{H}$ [56].

one below the $W^{+} W^{-}$production threshold. When $M_{H}$ is large enough to allow the production of a pair of gauge bosons, $H \rightarrow W^{+} W^{-}$and $H \rightarrow Z Z$ become dominant. For $M_{H}>2 m_{t}$, the $H \rightarrow t \bar{t}$ decay width is also sizeable, although smaller than the $W W$ and $Z Z$ ones because of the different dependence of the corresponding Higgs coupling with the mass scale (linear instead of quadratic). The design of the LHC detectors has taken into account all these very characteristic properties in order to optimize the search for the Higgs boson.

The neutral Higgs boson can also decay into two photons through the radiative one-loop transition $H \rightarrow t \bar{t} \rightarrow \gamma \gamma$. Although this decay mode is quite suppressed, it plays an important role in the LHC Higgs search at small masses because of its clean signature, in comparison with the big backgrounds affecting the dominant $H \rightarrow b \bar{b}$ channel.

The total decay width of the Higgs grows with increasing values of $M_{H}$. The effect is very strong above the $W^{+} W^{-}$production threshold. A heavy Higgs becomes then very broad. At $M_{H} \sim 600 \mathrm{GeV}$, the width is around $100 \mathrm{GeV}$; while for $M_{H} \sim 1 \mathrm{TeV}, \Gamma_{H}$ is already of the same size as the Higgs mass itself.

At LEP the Higgs boson could be produced through its gauge coupling to the $Z$ in Eq. (83), i.e., $e^{+} e^{-} \rightarrow Z \rightarrow Z H$, with the most important decay channels being $H \rightarrow b \bar{b}$ and $H \rightarrow \tau^{+} \tau^{-}$. The region of very low Higgs masses was also explored through $H \rightarrow e^{+} e^{-}(Z \rightarrow \nu \bar{\nu})$ and $H \rightarrow$ all $\left(Z \rightarrow e^{+} e^{-}, \mu^{+} \mu^{-}\right)$[57]. The LEP searches did not find any conclusive evidence of a SM Higgs boson. Combining the data from the four detectors, a lower bound of $114.4 \mathrm{GeV}$ (95\% C.L.) was set on the Higgs mass [40].

Gluon fusion $(G G \rightarrow t \bar{t} \rightarrow H)$ is the main Higgs production channel at the LHC and the Tevatron. Other subdominant mechanisms are $W, Z$ bremsstrahlung $(W \rightarrow W H, Z \rightarrow Z H)$ and $W W, Z Z$ and $t \bar{t}$ fusion ( $W W \rightarrow H, Z Z \rightarrow H, t \bar{t} \rightarrow H)$. The Higgs boson has been searched for systematically in a broad range of masses, using the most appropriate final states for each kinematical regime. The combination of all available CDF and D0 results, based on luminosities ranging from 4.0 to $8.6 \mathrm{fb}^{-1}$ collected at the Tevatron $(\sqrt{s}=1.96 \mathrm{TeV})$, excludes at 95\% C.L. Higgs masses between 156 and $177 \mathrm{GeV}$ [58]. Stronger constraints have been recently obtained at the LHC $(\sqrt{s}=7 \mathrm{TeV})$, based on integrated luminosities of up to $4.9 \mathrm{fb}^{-1}$. ATLAS excludes the mass ranges from 112.7 to $115.5,131$ to 237 and 251 to $453 \mathrm{GeV}$ [59], while CMS excludes masses between 127 and $600 \mathrm{GeV}$ [60] (95\% C.L.). The experimental limits are in agreement with the indirect upper bound in Eq. (108), from the global 

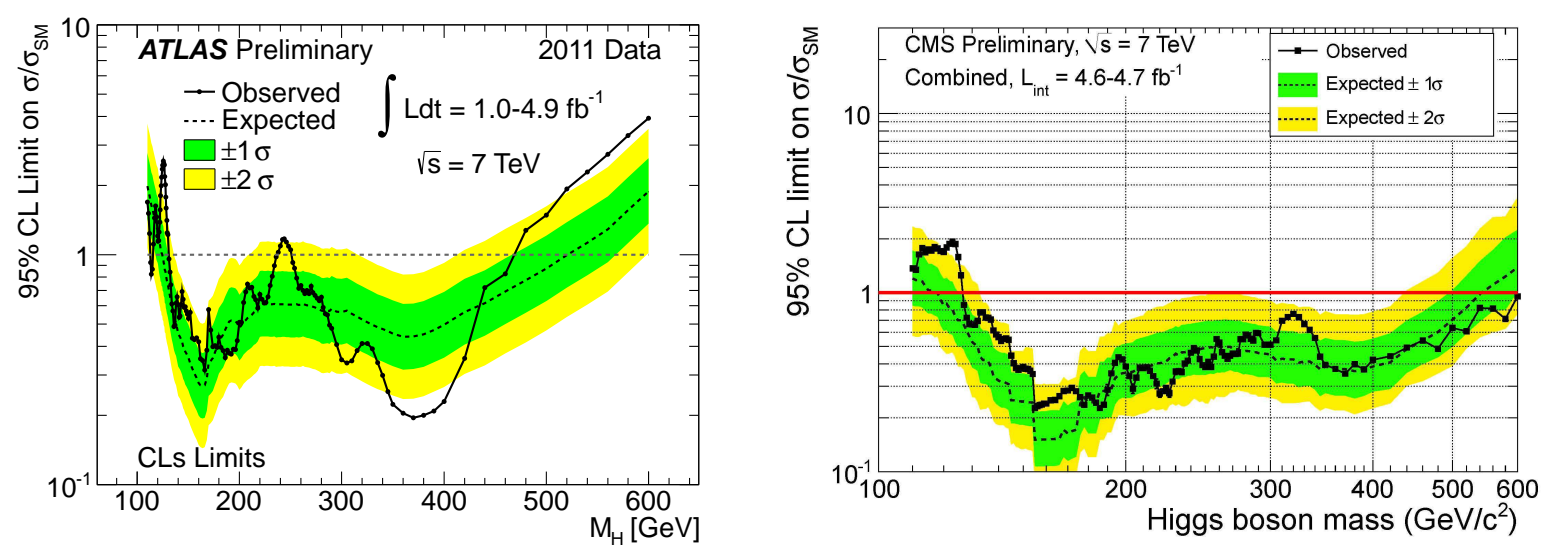

Fig. 29: Combined upper limits on the Higgs boson production cros-section divided by the SM prediction, as function of $M_{H}$, from ATLAS [59] (left) and CMS [60] (right). The dotted curve shows the expected limit in the absence of a signal and the bands indicate the corresponding $68 \%$ and $95 \%$ expected regions.

electroweak fit, reducing the range of possible SM Higgs boson masses to

$$
M_{H} \in[115.5,127] \mathrm{GeV} .
$$

As shown in Fig. 29, the present LHC limits are slightly weaker than expected (for the given luminosity) at low Higgs masses, due to an excess of events observed around $125 \mathrm{GeV}$ in different decay channels $\left(2 \gamma, Z Z^{*}, W W^{*}\right)[59,60]$. More data are required to clarify the origin of this excess.

\section{Flavour Dynamics}

We have learnt experimentally that there are six different quark flavours $u, d, s, c, b, t$, three different charged leptons $e, \mu, \tau$ and their corresponding neutrinos $\nu_{e}, \nu_{\mu}, \nu_{\tau}$. We can nicely include all these particles into the SM framework, by organizing them into three families of quarks and leptons, as indicated in Eqs. (1) and (2). Thus, we have three nearly identical copies of the same $S U(2)_{L} \otimes U(1)_{Y}$ structure, with masses as the only difference.

Let us consider the general case of $N_{G}$ generations of fermions, and denote $\nu_{j}^{\prime}, l_{j}^{\prime}, u_{j}^{\prime}, d_{j}^{\prime}$ the members of the weak family $j\left(j=1, \ldots, N_{G}\right)$, with definite transformation properties under the gauge group. Owing to the fermion replication, a large variety of fermion-scalar couplings are allowed by the gauge symmetry. The most general Yukawa Lagrangian has the form

$$
\begin{aligned}
& \mathcal{L}_{Y}=-\sum_{j k}\left\{\left(\bar{u}_{j}^{\prime}, \bar{d}^{\prime}{ }_{j}\right)_{L}\left[c_{j k}^{(d)}\left(\begin{array}{c}
\phi^{(+)} \\
\phi^{(0)}
\end{array}\right) d_{k R}^{\prime}+c_{j k}^{(u)}\left(\begin{array}{c}
\phi^{(0) *} \\
-\phi^{(-)}
\end{array}\right) u_{k R}^{\prime}\right]\right. \\
&\left.+\left(\bar{\nu}_{j}^{\prime}, \bar{l}_{j}^{\prime}\right)_{L} c_{j k}^{(l)}\left(\begin{array}{c}
\phi^{(+)} \\
\phi^{(0)}
\end{array}\right) l_{k R}^{\prime}\right\}+ \text { h.c. }
\end{aligned}
$$

where $c_{j k}^{(d)}, c_{j k}^{(u)}$ and $c_{j k}^{(l)}$ are arbitrary coupling constants.

In the unitary gauge, the Yukawa Lagrangian can be written as

$$
\mathcal{L}_{Y}=-\left(1+\frac{H}{v}\right)\left\{\overline{\mathbf{d}}_{L}^{\prime} \mathbf{M}_{d}^{\prime} \mathbf{d}_{R}^{\prime}+\overline{\mathbf{u}}_{L}^{\prime} \mathbf{M}_{u}^{\prime} \mathbf{u}_{R}^{\prime}+\overline{\mathbf{l}}_{L}^{\prime} \mathbf{M}_{l}^{\prime} \mathbf{l}_{R}^{\prime}+\text { h.c. }\right\} .
$$

Here, $\mathbf{d}^{\prime}, \mathbf{u}^{\prime}$ and $\mathbf{l}^{\prime}$ denote vectors in the $N_{G}$-dimensional flavour space, and the corresponding mass matrices are given by

$$
\left(\mathbf{M}_{d}^{\prime}\right)_{i j} \equiv c_{i j}^{(d)} \frac{v}{\sqrt{2}}, \quad\left(\mathbf{M}_{u}^{\prime}\right)_{i j} \equiv c_{i j}^{(u)} \frac{v}{\sqrt{2}}, \quad\left(\mathbf{M}_{l}^{\prime}\right)_{i j} \equiv c_{i j}^{(l)} \frac{v}{\sqrt{2}}
$$



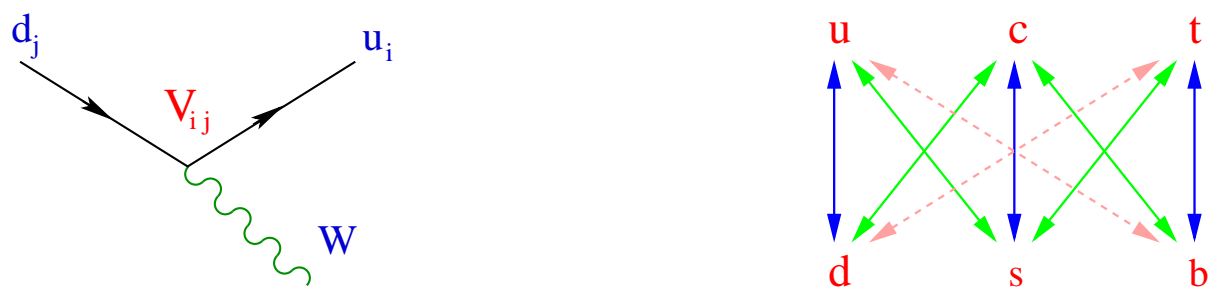

Fig. 30: Flavour-changing transitions through the charged-current couplings of the $W^{ \pm}$bosons.

The diagonalization of these mass matrices determines the mass eigenstates $d_{j}, u_{j}$ and $l_{j}$, which are linear combinations of the corresponding weak eigenstates $d_{j}^{\prime}, u_{j}^{\prime}$ and $l_{j}^{\prime}$, respectively.

The matrix $\mathbf{M}_{d}^{\prime}$ can be decomposed as $2 \mathbf{M}_{d}^{\prime}=\mathbf{H}_{d} \mathbf{U}_{d}=\mathbf{S}_{d}^{\dagger} \mathcal{M}_{d} \mathbf{S}_{d} \mathbf{U}_{d}$, where $\mathbf{H}_{d} \equiv \sqrt{\mathbf{M}_{d}^{\prime} \mathbf{M}_{d}^{\prime \dagger}}$ is an Hermitian positive-definite matrix, while $\mathbf{U}_{d}$ is unitary. $\mathbf{H}_{d}$ can be diagonalized by a unitary matrix $\mathbf{S}_{d}$; the resulting matrix $\mathcal{M}_{d}$ is diagonal, Hermitian and positive definite. Similarly, one has $\mathbf{M}_{u}^{\prime}=\mathbf{H}_{u} \mathbf{U}_{u}=\mathbf{S}_{u}^{\dagger} \mathcal{M}_{u} \mathbf{S}_{u} \mathbf{U}_{u}$ and $\mathbf{M}_{l}^{\prime}=\mathbf{H}_{l} \mathbf{U}_{l}=\mathbf{S}_{l}^{\dagger} \mathcal{M}_{l} \mathbf{S}_{l} \mathbf{U}_{l}$. In terms of the diagonal mass matrices

$$
\mathcal{M}_{d}=\operatorname{diag}\left(m_{d}, m_{s}, m_{b}, \ldots\right), \quad \mathcal{M}_{u}=\operatorname{diag}\left(m_{u}, m_{c}, m_{t}, \ldots\right), \quad \mathcal{M}_{l}=\operatorname{diag}\left(m_{e}, m_{\mu}, m_{\tau}, \ldots\right),
$$

the Yukawa Lagrangian takes the simpler form

$$
\mathcal{L}_{Y}=-\left(1+\frac{H}{v}\right)\left\{\overline{\mathbf{d}} \mathcal{M}_{d} \mathbf{d}+\overline{\mathbf{u}} \mathcal{M}_{u} \mathbf{u}+\overline{\mathbf{l}} \mathcal{M}_{l} \mathbf{l}\right\}
$$

where the mass eigenstates are defined by

$$
\begin{array}{lll}
\mathbf{d}_{L} \equiv \mathbf{S}_{d} \mathbf{d}_{L}^{\prime}, & \mathbf{u}_{L} \equiv \mathbf{S}_{u} \mathbf{u}_{L}^{\prime}, & \mathbf{l}_{L} \equiv \mathbf{S}_{l} \mathbf{l}_{L}^{\prime}, \\
\mathbf{d}_{R} \equiv \mathbf{S}_{d} \mathbf{U}_{d} \mathbf{d}_{R}^{\prime}, & \mathbf{u}_{R} \equiv \mathbf{S}_{u} \mathbf{U}_{u} \mathbf{u}_{R}^{\prime}, & \mathbf{l}_{R} \equiv \mathbf{S}_{l} \mathbf{U}_{l} \mathbf{l}_{R}^{\prime} .
\end{array}
$$

Note, that the Higgs couplings are proportional to the corresponding fermions masses.

Since, $\overline{\mathbf{f}}_{L}^{\prime} \mathbf{f}_{L}^{\prime}=\overline{\mathbf{f}}_{L} \mathbf{f}_{L}$ and $\overline{\mathbf{f}}_{R}^{\prime} \mathbf{f}_{R}^{\prime}=\overline{\mathbf{f}}_{R} \mathbf{f}_{R}(f=d, u, l)$, the form of the neutral-current part of the $S U(2)_{L} \otimes U(1)_{Y}$ Lagrangian does not change when expressed in terms of mass eigenstates. Therefore, there are no flavour-changing neutral currents in the SM (GIM mechanism [5]). This is a consequence of treating all equal-charge fermions on the same footing.

However, $\overline{\mathbf{u}}_{L}^{\prime} \mathbf{d}_{L}^{\prime}=\overline{\mathbf{u}}_{L} \mathbf{S}_{u} \mathbf{S}_{d}^{\dagger} \mathbf{d}_{L} \equiv \overline{\mathbf{u}}_{L} \mathbf{V} \mathbf{d}_{L}$. In general, $\mathbf{S}_{u} \neq \mathbf{S}_{d}$; thus, if one writes the weak eigenstates in terms of mass eigenstates, a $N_{G} \times N_{G}$ unitary mixing matrix $\mathbf{V}$, called the CabibboKobayashi-Maskawa (CKM) matrix [61,62], appears in the quark charged-current sector:

$$
\mathcal{L}_{\mathrm{CC}}=-\frac{g}{2 \sqrt{2}}\left\{W_{\mu}^{\dagger}\left[\sum_{i j} \bar{u}_{i} \gamma^{\mu}\left(1-\gamma_{5}\right) \mathbf{V}_{i j} d_{j}+\sum_{l} \bar{\nu}_{l} \gamma^{\mu}\left(1-\gamma_{5}\right) l\right]+\text { h.c. }\right\} .
$$

The matrix $\mathbf{V}$ couples any 'up-type' quark with all 'down-type' quarks (Fig. 30).

If neutrinos are assumed to be massless, we can always redefine the neutrino flavours, in such a way as to eliminate the analogous mixing in the lepton sector: $\overline{\boldsymbol{\nu}}_{L}^{\prime} \mathbf{l}_{L}^{\prime}=\overline{\boldsymbol{\nu}}_{L}^{\prime} \mathbf{S}_{l}^{\dagger} \mathbf{l}_{L} \equiv \overline{\boldsymbol{\nu}}_{L} \mathbf{l}_{L}$. Thus, we have lepton-flavour conservation in the minimal SM without right-handed neutrinos. If sterile $\nu_{R}$ fields

\footnotetext{
${ }^{2}$ The condition $\operatorname{det} \mathbf{M}_{f}^{\prime} \neq 0(f=d, u, l)$ guarantees that the decomposition $\mathbf{M}_{f}^{\prime}=\mathbf{H}_{f} \mathbf{U}_{f}$ is unique: $\mathbf{U}_{f} \equiv \mathbf{H}_{f}^{-1} \mathbf{M}_{f}^{\prime}$. The matrices $\mathbf{S}_{f}$ are completely determined (up to phases) only if all diagonal elements of $\mathbf{M}_{f}$ are different. If there is some degeneracy, the arbitrariness of $\mathbf{S}_{f}$ reflects the freedom to define the physical fields. If $\operatorname{det} \mathbf{M}_{f}^{\prime}=0$, the matrices $\mathbf{U}_{f}$ and $\mathbf{S}_{f}$ are not uniquely determined, unless their unitarity is explicitly imposed.
} 
are included in the model, one has an additional Yukawa term in Eq. (110), giving rise to a neutrino mass matrix $\left(\mathbf{M}_{\nu}^{\prime}\right)_{i j} \equiv c_{i j}^{(\nu)} v / \sqrt{2}$. Thus, the model can accommodate non-zero neutrino masses and lepton-flavour violation through a lepton mixing matrix $\mathbf{V}_{L}$ analogous to the one present in the quark sector. Note, however, that the total lepton number $L \equiv L_{e}+L_{\mu}+L_{\tau}$ is still conserved. We know experimentally that neutrino masses are tiny and there are strong bounds on lepton-flavour violating decays: $\operatorname{Br}\left(\mu^{ \pm} \rightarrow e^{ \pm} e^{+} e^{-}\right)<1.0 \cdot 10^{-12}$ [63], $\operatorname{Br}\left(\mu^{ \pm} \rightarrow e^{ \pm} \gamma\right)<2.4 \cdot 10^{-12}$ [64], $\operatorname{Br}\left(\tau^{ \pm} \rightarrow \mu^{ \pm} \gamma\right)<$ $4.4 \cdot 10^{-8}$ [65, 66] ... However, we do have a clear evidence of neutrino oscillation phenomena.

The fermion masses and the quark mixing matrix $\mathbf{V}$ are all determined by the Yukawa couplings in Eq. (110). However, the coefficients $c_{i j}^{(f)}$ are not known; therefore we have a bunch of arbitrary parameters. A general $N_{G} \times N_{G}$ unitary matrix is characterized by $N_{G}^{2}$ real parameters: $N_{G}\left(N_{G}-1\right) / 2$ moduli and $N_{G}\left(N_{G}+1\right) / 2$ phases. In the case of $\mathbf{V}$, many of these parameters are irrelevant, because we can always choose arbitrary quark phases. Under the phase redefinitions $u_{i} \rightarrow \mathrm{e}^{i \phi_{i}} u_{i}$ and $d_{j} \rightarrow$ $\mathrm{e}^{i \theta_{j}} d_{j}$, the mixing matrix changes as $\mathbf{V}_{i j} \rightarrow \mathbf{V}_{i j} \mathrm{e}^{i\left(\theta_{j}-\phi_{i}\right)}$; thus, $2 N_{G}-1$ phases are unobservable. The number of physical free parameters in the quark-mixing matrix then gets reduced to $\left(N_{G}-1\right)^{2}$ : $N_{G}\left(N_{G}-1\right) / 2$ moduli and $\left(N_{G}-1\right)\left(N_{G}-2\right) / 2$ phases.

In the simpler case of two generations, $\mathbf{V}$ is determined by a single parameter. One then recovers the Cabibbo rotation matrix [61]

$$
\mathbf{V}=\left(\begin{array}{cc}
\cos \theta_{C} & \sin \theta_{C} \\
-\sin \theta_{C} & \cos \theta_{C}
\end{array}\right)
$$

With $N_{G}=3$, the CKM matrix is described by three angles and one phase. Different (but equivalent) representations can be found in the literature. The Particle data Group [9] advocates the use of the following one as the 'standard' CKM parametrization:

$$
\mathbf{V}=\left[\begin{array}{ccc}
c_{12} c_{13} & s_{12} c_{13} & s_{13} \mathrm{e}^{-i \delta_{13}} \\
-s_{12} c_{23}-c_{12} s_{23} s_{13} \mathrm{e}^{i \delta_{13}} & c_{12} c_{23}-s_{12} s_{23} s_{13} \mathrm{e}^{i \delta_{13}} & s_{23} c_{13} \\
s_{12} s_{23}-c_{12} c_{23} s_{13} \mathrm{e}^{i \delta_{13}} & -c_{12} s_{23}-s_{12} c_{23} s_{13} \mathrm{e}^{i \delta_{13}} & c_{23} c_{13}
\end{array}\right]
$$

Here $c_{i j} \equiv \cos \theta_{i j}$ and $s_{i j} \equiv \sin \theta_{i j}$, with $i$ and $j$ being 'generation' labels $(i, j=1,2,3)$. The real angles $\theta_{12}, \theta_{23}$ and $\theta_{13}$ can all be made to lie in the first quadrant, by an appropriate redefinition of quark field phases; then, $c_{i j} \geq 0, s_{i j} \geq 0$ and $0 \leq \delta_{13} \leq 2 \pi$.

Notice that $\delta_{13}$ is the only complex phase in the SM Lagrangian. Therefore, it is the only possible source of $\mathcal{C} \mathcal{P}$-violation phenomena. In fact, it was for this reason that the third generation was assumed to exist [62], before the discovery of the $b$ and the $\tau$. With two generations, the SM could not explain the observed $\mathcal{C P}$ violation in the $K$ system.

\subsection{Quark mixing}

Our knowledge of the charged-current parameters is unfortunately not so good as in the neutral-current case. In order to measure the CKM matrix elements, one needs to study hadronic weak decays of the type $H \rightarrow H^{\prime} l^{-} \bar{\nu}_{l}$ or $H \rightarrow H^{\prime} l^{+} \nu_{l}$, which are associated with the corresponding quark transitions $d_{j} \rightarrow u_{i} l^{-} \bar{\nu}_{l}$ and $u_{i} \rightarrow d_{j} l^{+} \nu_{l}$ (Fig. 31). Since quarks are confined within hadrons, the decay amplitude

$$
T\left[H \rightarrow H^{\prime} l^{-} \bar{\nu}_{l}\right] \approx \frac{G_{F}}{\sqrt{2}} \mathbf{V}_{i j}\left\langle H^{\prime}\left|\bar{u}_{i} \gamma^{\mu}\left(1-\gamma_{5}\right) d_{j}\right| H\right\rangle\left[\bar{l} \gamma_{\mu}\left(1-\gamma_{5}\right) \nu_{l}\right]
$$

always involves an hadronic matrix element of the weak left current. The evaluation of this matrix element is a non-perturbative QCD problem, which introduces unavoidable theoretical uncertainties.

One usually looks for a semileptonic transition where the matrix element can be fixed at some kinematical point by a symmetry principle. This has the virtue of reducing the theoretical uncertainties 

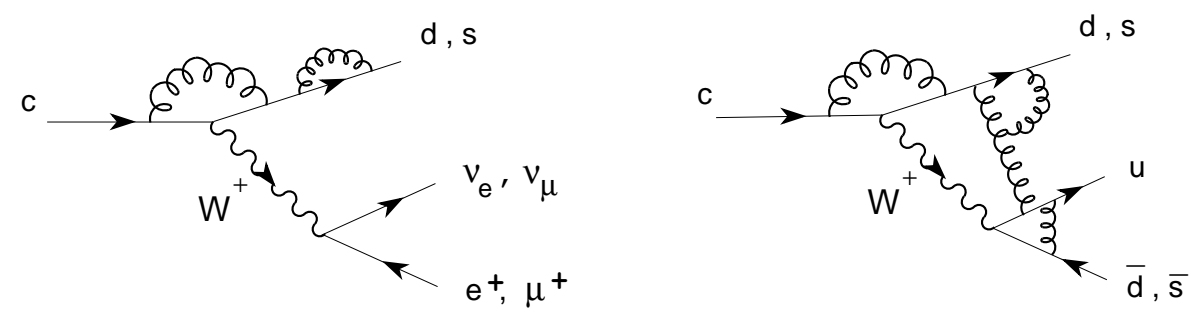

Fig. 31: Determinations of $\mathbf{V}_{i j}$ are done in semileptonic quark decays (left), where a single quark current is present. Hadronic decay modes (right) involve two different quark currents and are more affected by QCD effects (gluons can couple everywhere).

to the level of symmetry-breaking corrections and kinematical extrapolations. The standard example is a $0^{-} \rightarrow 0^{-}$decay such as $K \rightarrow \pi l \nu, D \rightarrow K l \nu$ or $B \rightarrow D l \nu$. Only the vector current can contribute in this case:

$$
\left\langle P^{\prime}\left(k^{\prime}\right)\left|\bar{u}_{i} \gamma^{\mu} d_{j}\right| P(k)\right\rangle=C_{P P^{\prime}}\left\{\left(k+k^{\prime}\right)^{\mu} f_{+}(t)+\left(k-k^{\prime}\right)^{\mu} f_{-}(t)\right\} .
$$

Here, $C_{P P^{\prime}}$ is a Clebsh-Gordan factor and $t=\left(k-k^{\prime}\right)^{2} \equiv q^{2}$. The unknown strong dynamics is fully contained in the form factors $f_{ \pm}(t)$. In the limit of equal quark masses, $m_{u_{i}}=m_{d_{j}}$, the divergence of the vector current is zero; thus $q_{\mu}\left[\bar{u}_{i} \gamma^{\mu} d_{j}\right]=0$, which implies $f_{-}(t)=0$ and, moreover, $f_{+}(0)=1$ to all orders in the strong coupling because the associated flavour charge is a conserved quantity 3 Therefore, one only needs to estimate the corrections induced by the quark mass differences.

Since $q^{\mu}\left[\bar{l} \gamma_{\mu}\left(1-\gamma_{5}\right) \nu_{l}\right] \sim m_{l}$, the contribution of $f_{-}(t)$ is kinematically suppressed in the electron and muon modes. The decay width can then be written as

$$
\Gamma\left(P \rightarrow P^{\prime} l \nu\right)=\frac{G_{F}^{2} M_{P}^{5}}{192 \pi^{3}}\left|\mathbf{V}_{i j}\right|^{2} C_{P P^{\prime}}^{2}\left|f_{+}(0)\right|^{2} \mathcal{I}\left(1+\delta_{\mathrm{RC}}\right),
$$

where $\delta_{\mathrm{RC}}$ is an electroweak radiative correction factor and $\mathcal{I}$ denotes a phase-space integral, which in the $m_{l}=0$ limit takes the form

$$
\mathcal{I} \approx \int_{0}^{\left(M_{P}-M_{P^{\prime}}\right)^{2}} \frac{d t}{M_{P}^{8}} \lambda^{3 / 2}\left(t, M_{P}^{2}, M_{P^{\prime}}^{2}\right)\left|\frac{f_{+}(t)}{f_{+}(0)}\right|^{2} .
$$

The usual procedure to determine $\left|\mathbf{V}_{i j}\right|$ involves three steps:

1. Measure the shape of the $t$ distribution. This fixes $\left|f_{+}(t) / f_{+}(0)\right|$ and therefore determines $\mathcal{I}$.

2. Measure the total decay width $\Gamma$. Since $G_{F}$ is already known from $\mu$ decay, one gets then an experimental value for the product $\left|f_{+}(0)\right|\left|\mathbf{V}_{i j}\right|$.

3. Get a theoretical prediction for $f_{+}(0)$.

It is important to realize that theoretical input is always needed. Thus, the accuracy of the $\left|\mathbf{V}_{i j}\right|$ determination is limited by our ability to calculate the relevant hadronic parameters.

The conservation of the vector and axial-vector QCD currents in the massless quark limit allows for accurate determinations of the light-quark mixings $\left|\mathbf{V}_{u d}\right|$ and $\left|\mathbf{V}_{u s}\right|$. The present values are shown in Table 4. Since $\left|\mathbf{V}_{u b}\right|^{2}$ is tiny, these two light quark entries provide a sensible test of the unitarity of the CKM matrix:

$$
\left|\mathbf{V}_{u d}\right|^{2}+\left|\mathbf{V}_{u s}\right|^{2}+\left|\mathbf{V}_{u b}\right|^{2}=1.0000 \pm 0.0007
$$

\footnotetext{
${ }^{3}$ This is completely analogous to the electromagnetic charge conservation in QED. The conservation of the electromagnetic current implies that the proton electromagnetic form factor does not get any QED or QCD correction at $q^{2}=0$ and, therefore, $Q(p)=2 Q(u)+Q(d)=|Q(e)|$. A detailed proof can be found in Ref. [67].
} 
Table 4: Direct determinations of the CKM matrix elements $\mathbf{V}_{i j}$ [41]. The 'best' values are indicated in bold face.

\begin{tabular}{|c|c|c|}
\hline CKM entry & Value & Source \\
\hline \multirow[t]{3}{*}{$\left|\mathbf{V}_{u d}\right|$} & $0.97425 \pm 0.00022$ & Nuclear $\beta$ decay [68] \\
\hline & $0.9765 \pm 0.0018$ & $n \rightarrow p e^{-} \bar{\nu}_{e}$ \\
\hline & $0.9741 \pm 0.0026$ & $\pi^{+} \rightarrow \pi^{0} e^{+} \nu_{e}$ [71,72] \\
\hline \multirow{4}{*}{$\left|\mathbf{V}_{u s}\right|$} & $0.2255 \pm 0.0013$ & $K \rightarrow \pi l^{+} \nu_{l}[73,74$ \\
\hline & $0.2256 \pm 0.0012$ & $K^{+} / \pi^{+} \rightarrow \mu^{+} \nu_{\mu}, \mathbf{V}_{u d}$ \\
\hline & $0.2166 \pm 0.0020$ & $\tau$ decays \\
\hline & $0.226 \pm 0.005$ & Hyperon decays [78,79] \\
\hline \multirow[t]{2}{*}{$\left|\mathbf{V}_{c d}\right|$} & $0.230 \pm 0.011$ & $\nu d \rightarrow c X$ \\
\hline & $0.234 \pm 0.026$ & $D \rightarrow \pi l \bar{\nu}_{l}$ \\
\hline \multirow[t]{3}{*}{$\left|\mathbf{V}_{c s}\right|$} & $0.963 \pm 0.026$ & $D \rightarrow K l \bar{\nu}_{l} \quad 80,81$ \\
\hline & $0.944_{-0.29}^{+0.35}$ & $W^{+} \rightarrow c \bar{s}$ \\
\hline & $0.973 \pm 0.014$ & $W^{+} \rightarrow$ had. $, \mathbf{V}_{u j}, \mathbf{V}_{c d}, \mathbf{V}_{c b}$ \\
\hline \multirow[t]{3}{*}{$\left|\mathbf{V}_{c b}\right|$} & $0.0396 \pm 0.0008$ & $B \rightarrow D^{*} l \bar{\nu}_{l}, D l \bar{\nu}_{l}$ \\
\hline & $0.04185 \pm 0.00073$ & $b \rightarrow c l \bar{\nu}_{l}$ \\
\hline & $0.0408 \pm 0.0011$ & Average \\
\hline \multirow[t]{3}{*}{$\left|\mathbf{V}_{u b}\right|$} & $0.00338 \pm 0.00036$ & $B \rightarrow \pi l \bar{\nu}_{l}$ \\
\hline & $0.00427 \pm 0.00038$ & $b \rightarrow u l \bar{\nu}_{l}$ \\
\hline & $0.00389 \pm 0.00044$ & Average \\
\hline$\left|\mathbf{V}_{t b}\right| / \sqrt{\sum_{q}\left|\mathbf{V}_{t q}\right|^{2}}$ & $>0.89(95 \% \mathrm{CL})$ & $t \rightarrow b W / q W$ \\
\hline$\left|\mathbf{V}_{t b}\right|$ & $0.88 \pm 0.07$ & $p \bar{p} \rightarrow t \bar{b}+X$ \\
\hline
\end{tabular}

It is important to notice that at the quoted level of uncertainty radiative corrections play a crucial role.

In the limit of very heavy quark masses, QCD has additional symmetries [89-92] which can be used to make rather precise determinations of $\left|\mathbf{V}_{c b}\right|$, either from exclusive decays such as $B \rightarrow D^{*} l \bar{\nu}_{l}$ [93, 94] or from the inclusive analysis of $b \rightarrow c l \bar{\nu}_{l}$ transitions. At present there is a slight discrepancy between the exclusive and inclusive determinations which is reflected in the larger error quoted for their average in Table 4, following the PDG prescription [9]. A similar disagreement is observed for the analogous $\left|\mathbf{V}_{u b}\right|$ determinations. The control of theoretical uncertainties is much more difficult for $\left|\mathbf{V}_{u b}\right|$, $\left|\mathbf{V}_{c d}\right|$ and $\left|\mathbf{V}_{c s}\right|$, because the symmetry arguments associated with the light and heavy quark limits get corrected by sizeable symmetry-breaking effects.

The most precise determination of $\left|\mathbf{V}_{c d}\right|$ is based on neutrino and antineutrino interactions. The difference of the ratio of double-muon to single-muon production by neutrino and antineutrino beams is proportional to the charm cross-section off valence $d$ quarks and, therefore, to $\left|\mathbf{V}_{c d}\right|$. A direct determination of $\left|\mathbf{V}_{c s}\right|$ can be also obtained from charm-tagged $W$ decays at LEP2. Moreover, the ratio of the total hadronic decay width of the $W$ to the leptonic one provides the sum [34,35]

$$
\sum_{\substack{i=u, c \\ j=d, s, b}}\left|\mathbf{V}_{i j}\right|^{2}=2.002 \pm 0.027 .
$$


Although much less precise than Eq. (123), this result test unitarity at the $1.3 \%$ level. From Eq. (124) one can also obtain a tighter determination of $\left|\mathbf{V}_{c s}\right|$, using the experimental knowledge on the other CKM matrix elements, i.e., $\left|\mathbf{V}_{u d}\right|^{2}+\left|\mathbf{V}_{u s}\right|^{2}+\left|\mathbf{V}_{u b}\right|^{2}+\left|\mathbf{V}_{c d}\right|^{2}+\left|\mathbf{V}_{c b}\right|^{2}=1.0546 \pm 0.0051$. This gives the most accurate and final value of $\left|\mathbf{V}_{c s}\right|$ quoted in Table 4 ,

The measured entries of the CKM matrix show a hierarchical pattern, with the diagonal elements being very close to one, the ones connecting the two first generations having a size

$$
\lambda \approx\left|\mathbf{V}_{u s}\right|=0.2255 \pm 0.0013
$$

the mixing between the second and third families being of order $\lambda^{2}$, and the mixing between the first and third quark generations having a much smaller size of about $\lambda^{3}$. It is then quite practical to use the approximate parametrization [95]:

$$
\mathbf{V}=\left[\begin{array}{ccc}
1-\frac{\lambda^{2}}{2} & \lambda & A \lambda^{3}(\rho-i \eta) \\
-\lambda & 1-\frac{\lambda^{2}}{2} & A \lambda^{2} \\
A \lambda^{3}(1-\rho-i \eta) & -A \lambda^{2} & 1
\end{array}\right]+O\left(\lambda^{4}\right)
$$

where

$$
A \approx \frac{\left|\mathbf{V}_{c b}\right|}{\lambda^{2}}=0.802 \pm 0.024, \quad \sqrt{\rho^{2}+\eta^{2}} \approx\left|\frac{\mathbf{V}_{u b}}{\lambda \mathbf{V}_{c b}}\right|=0.423 \pm 0.049
$$

Defining to all orders in $\lambda\left[\right.$ [96] $s_{12} \equiv \lambda, s_{23} \equiv A \lambda^{2}$ and $s_{13} \mathrm{e}^{-i \delta_{13}} \equiv A \lambda^{3}(\rho-i \eta)$, Eq. [126) just corresponds to a Taylor expansion of Eq. (118) in powers of $\lambda$.

\subsection{CP Violation}

While parity and charge conjugation are violated by the weak interactions in a maximal way, the product of the two discrete transformations is still a good symmetry of the gauge interactions (left-handed fermions $\leftrightarrow$ right-handed antifermions). In fact, $\mathcal{C P}$ appears to be a symmetry of nearly all observed phenomena. However, a slight violation of the $\mathcal{C P}$ symmetry at the level of $0.2 \%$ is observed in the neutral kaon system and more sizeable signals of $\mathcal{C P}$ violation have been recently established at the $\mathrm{B}$ factories. Moreover, the huge matter-antimatter asymmetry present in our Universe is a clear manifestation of $\mathcal{C P}$ violation and its important role in the primordial baryogenesis.

The $\mathcal{C P} \mathcal{T}$ theorem guarantees that the product of the three discrete transformations is an exact symmetry of any local and Lorentz-invariant quantum field theory preserving micro-causality. Therefore, a violation of $\mathcal{C P}$ requires a corresponding violation of time reversal. Since $\mathcal{T}$ is an antiunitary transformation, this requires the presence of relative complex phases between different interfering amplitudes.

The electroweak SM Lagrangian only contains a single complex phase $\delta_{13}(\eta)$. This is the sole possible source of $\mathcal{C P}$ violation and, therefore, the SM predictions for $\mathcal{C P}$-violating phenomena are quite constrained. The CKM mechanism requires several necessary conditions in order to generate an observable $\mathcal{C} \mathcal{P}$-violation effect. With only two fermion generations, the quark mixing mechanism cannot give rise to $\mathcal{C P}$ violation; therefore, for $\mathcal{C P}$ violation to occur in a particular process, all three generations are required to play an active role. In the kaon system, for instance, $\mathcal{C P}$-violation effects can only appear at the one-loop level, where the top quark is present. In addition, all CKM matrix elements must be nonzero and the quarks of a given charge must be non-degenerate in mass. If any of these conditions were not satisfied, the CKM phase could be rotated away by a redefinition of the quark fields. $\mathcal{C P}$-violation effects are then necessarily proportional to the product of all CKM angles, and should vanish in the limit where any two (equal-charge) quark masses are taken to be equal. All these necessary conditions can be 
summarized as a single requirement on the original quark mass matrices $\mathbf{M}_{u}^{\prime}$ and $\mathbf{M}_{d}^{\prime}$ [97]:

$$
\mathcal{C P} \text { violation } \quad \Longleftrightarrow \quad \operatorname{Im}\left\{\operatorname{det}\left[\mathbf{M}_{u}^{\prime} \mathbf{M}_{u}^{\prime \dagger}, \mathbf{M}_{d}^{\prime} \mathbf{M}_{d}^{\prime \dagger}\right]\right\} \neq 0
$$

Without performing any detailed calculation, one can make the following general statements on the implications of the CKM mechanism of $\mathcal{C P}$ violation:

- Owing to unitarity, for any choice of $i, j, k, l$ (between 1 and 3),

$$
\begin{gathered}
\operatorname{Im}\left[\mathbf{V}_{i j} \mathbf{V}_{i k}^{*} \mathbf{V}_{l k} \mathbf{V}_{l j}^{*}\right]=\mathcal{J} \sum_{m, n=1}^{3} \epsilon_{i l m} \epsilon_{j k n}, \\
\mathcal{J}=c_{12} c_{23} c_{13}^{2} s_{12} s_{23} s_{13} \sin \delta_{13} \approx A^{2} \lambda^{6} \eta<10^{-4} .
\end{gathered}
$$

Any $\mathcal{C P}$-violation observable involves the product $\mathcal{J}$ [97]. Thus, violations of the $\mathcal{C P}$ symmetry are necessarily small.

- In order to have sizeable $\mathcal{C} \mathcal{P}$-violating asymmetries $\mathcal{A} \equiv(\Gamma-\bar{\Gamma}) /(\Gamma+\bar{\Gamma})$, one should look for very suppressed decays, where the decay widths already involve small CKM matrix elements.

- In the SM, $\mathcal{C P}$ violation is a low-energy phenomenon, in the sense that any effect should disappear when the quark mass difference $m_{c}-m_{u}$ becomes negligible.

- $B$ decays are the optimal place for $\mathcal{C P}$-violation signals to show up. They involve small CKM matrix elements and are the lowest-mass processes where the three quark generations play a direct (tree-level) role.

The SM mechanism of $\mathcal{C P}$ violation is based on the unitarity of the CKM matrix. Testing the constraints implied by unitarity is then a way to test the source of $\mathcal{C P}$ violation. The unitarity tests in Eqs. (123) and (124) involve only the moduli of the CKM parameters, while $\mathcal{C P}$ violation has to do with their phases. More interesting are the off-diagonal unitarity conditions:

$$
\begin{aligned}
\mathbf{V}_{u d}^{*} \mathbf{V}_{u s}+\mathbf{V}_{c d}^{*} \mathbf{V}_{c s}+\mathbf{V}_{t d}^{*} \mathbf{V}_{t s} & =0 \\
\mathbf{V}_{u s}^{*} \mathbf{V}_{u b}+\mathbf{V}_{c s}^{*} \mathbf{V}_{c b}+\mathbf{V}_{t s}^{*} \mathbf{V}_{t b} & =0 \\
\mathbf{V}_{u b}^{*} \mathbf{V}_{u d}+\mathbf{V}_{c b}^{*} \mathbf{V}_{c d}+\mathbf{V}_{t b}^{*} \mathbf{V}_{t d} & =0
\end{aligned}
$$

These relations can be visualized by triangles in a complex plane which, owing to Eq. (129), have the same area $|\mathcal{J}| / 2$. In the absence of $\mathcal{C P}$ violation, these triangles would degenerate into segments along the real axis.

In the first two triangles, one side is much shorter than the other two (the Cabibbo suppression factors of the three sides are $\lambda, \lambda$ and $\lambda^{5}$ in the first triangle, and $\lambda^{4}, \lambda^{2}$ and $\lambda^{2}$ in the second one). This is why $\mathcal{C P}$ effects are so small for $K$ mesons (first triangle), and why certain asymmetries in $B_{s}$ decays are predicted to be tiny (second triangle). The third triangle looks more interesting, since the three sides have a similar size of about $\lambda^{3}$. They are small, which means that the relevant $b$-decay branching ratios are small, but once enough $B$ mesons have been produced, the $\mathcal{C} \mathcal{P}$-violation asymmetries are sizeable. The present experimental constraints on this triangle are shown in Fig. 32, where it has been scaled by dividing its sides by $\mathbf{V}_{c b}^{*} \mathbf{V}_{c d}$. This aligns one side of the triangle along the real axis and makes its length equal to 1 ; the coordinates of the 3 vertices are then $(0,0),(1,0)$ and $(\bar{\rho}, \bar{\eta}) \equiv\left(1-\lambda^{2} / 2\right)(\rho, \eta)$.

One side of the unitarity triangle has been already determined in Eq. (127) from the ratio $\left|\mathbf{V}_{u b} / \mathbf{V}_{c b}\right|$. The other side can be obtained from the measured mixing between the $B_{d}^{0}$ and $\bar{B}_{d}^{0}$ mesons (Fig. 33), $\Delta M_{d}=0.507 \pm 0.004 \mathrm{ps}^{-1}$ [83], which fixes $\left|\mathbf{V}_{t b}\right|$. Additional information has been provided by the observation of $B_{s}^{0}-\bar{B}_{s}^{0}$ oscillations at CDF and LHCb, implying $\Delta M_{s}=17.69 \pm 0.08 \mathrm{ps}^{-1}$ [98, 99]. 


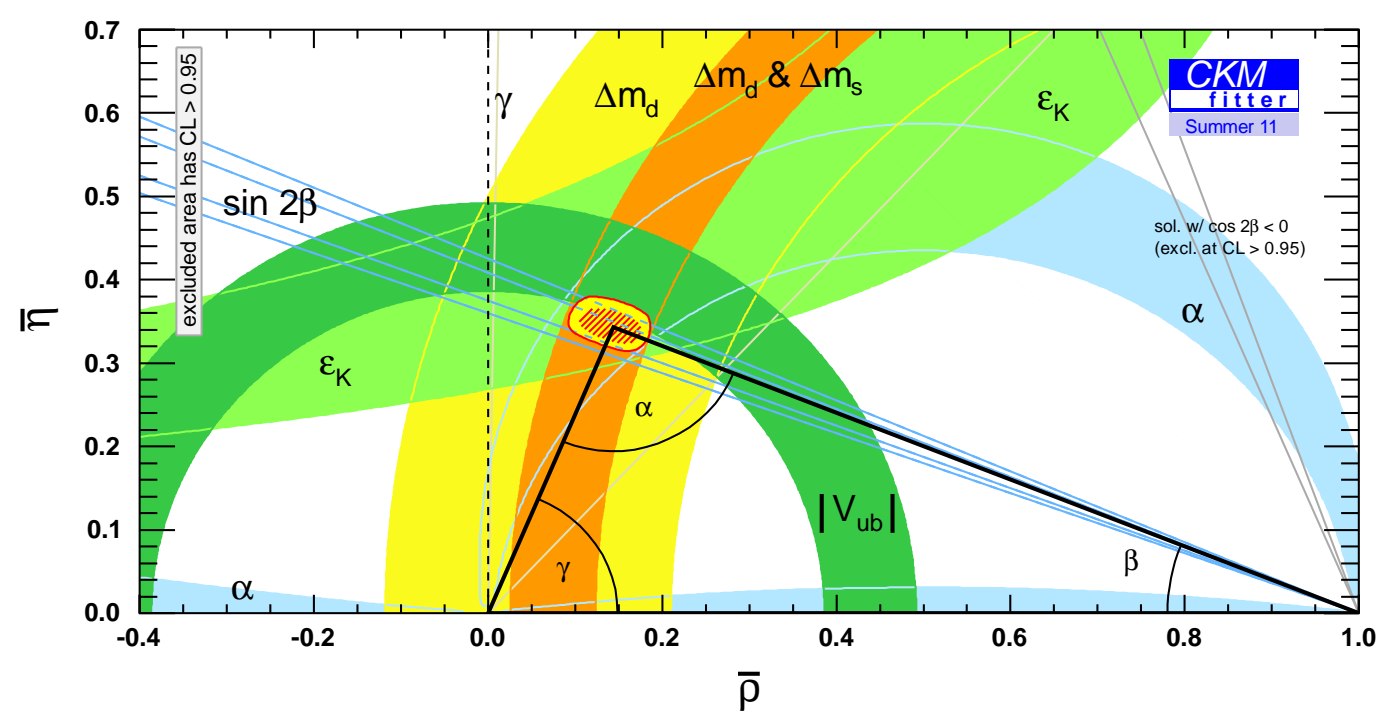

Fig. 32: Experimental constraints on the SM unitarity triangle [100].
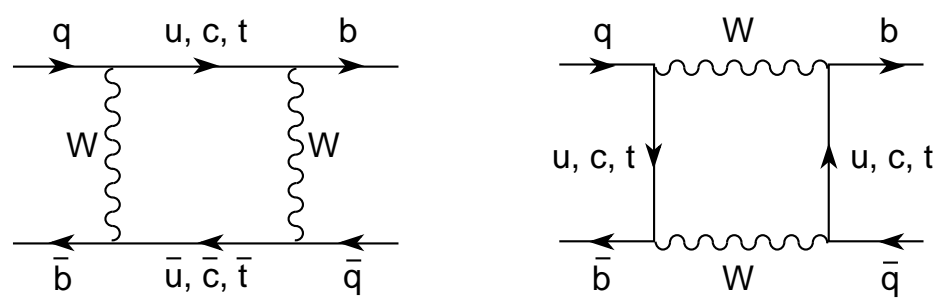

Fig. 33: $B^{0}-\bar{B}^{0}$ mixing diagrams. Owing to the unitarity of the CKM matrix, the mixing vanishes for equal uptype quark masses (GIM mechanism). The mixing amplitude is then proportional to the mass (squared) splittings between the $u, c$ and $t$ quarks, and is completely dominated by the top contribution.

From the experimental ratio $\Delta M_{d} / \Delta M_{s}=0.0287 \pm 0.0003$, one obtains $\left|\mathbf{V}_{t d}\right| /\left|\mathbf{V}_{t s}\right|$. A more direct constraint on the parameter $\eta$ is given by the observed $\mathcal{C P}$ violation in $K^{0} \rightarrow 2 \pi$ decays. The measured value of $\left|\varepsilon_{K}\right|=(2.228 \pm 0.011) \cdot 10^{-3}$ [9] determines the parabolic region shown in Fig. 32 ]

$B^{0}$ decays into $\mathcal{C P}$ self-conjugate final states provide independent ways to determine the angles of the unitarity triangle [101, 102]. The $B^{0}$ (or $\bar{B}^{0}$ ) can decay directly to the given final state $f$, or do it after the meson has been changed to its antiparticle via the mixing process. $\mathcal{C} \mathcal{P}$-violating effects can then result from the interference of these two contributions. The time-dependent $\mathcal{C P}$-violating rate asymmetries contain direct information on the CKM parameters. The gold-plated decay mode is $B_{d}^{0} \rightarrow$ $J / \psi K_{S}$, which gives a clean measurement of $\beta \equiv-\arg \left(\mathbf{V}_{c d} \mathbf{V}_{c b}^{*} / \mathbf{V}_{t d} \mathbf{V}_{t b}^{*}\right)$, without strong-interaction uncertainties. Including the information obtained from other $b \rightarrow c \bar{c} s$ decays, one gets [83]:

$$
\sin (2 \beta)=0.68 \pm 0.02 .
$$

Many additional tests of the CKM matrix from different $B$ decay modes are being pursued with the large data samples collected at the $B$ factories. Determinations of the other two angles of the unitarity triangle, $\alpha \equiv-\arg \left(\mathbf{V}_{t d} \mathbf{V}_{t b}^{*} / \mathbf{V}_{u d} \mathbf{V}_{u b}^{*}\right)$ and $\gamma \equiv-\arg \left(\mathbf{V}_{u d} \mathbf{V}_{u b}^{*} / \mathbf{V}_{c d} \mathbf{V}_{c b}^{*}\right)$, have been already obtained [83], and are included in the global fit shown in Fig. 32 [100, 103]. The different sets of data fit nicely, providing a quite accurate determination of the unitarity triangle vertex $(\bar{\rho}, \bar{\eta})$. Complementary and very valuable information could be also obtained from the kaon decay modes $K^{ \pm} \rightarrow \pi^{ \pm} \nu \bar{\nu}, K_{L} \rightarrow \pi^{0} \nu \bar{\nu}$ and $K_{L} \rightarrow \pi^{0} e^{+} e^{-}$[74, 104]. A more detailed overview of flavour physics is given in Ref. [41]. 


\subsection{Lepton mixing}

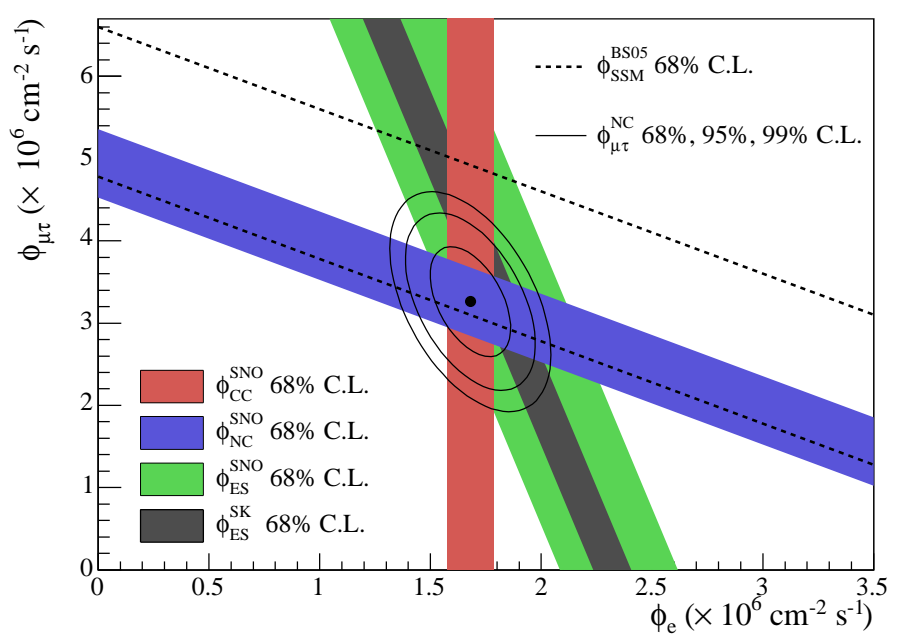

Fig. 34: Measured fluxes of ${ }^{8} B$ solar neutrinos of $\nu_{\mu}$ or $\nu_{\tau}$ type $\left(\phi_{\mu, \tau}\right)$ versus the flux of $\nu_{e}\left(\phi_{e}\right)$ [105].

The so-called 'solar neutrino problem' has been a long-standing question, since the very first chlorine experiment at the Homestake mine [106]. The flux of solar $\nu_{e}$ neutrinos reaching the Earth has been measured by several experiments to be significantly below the standard solar model prediction [107]. More recently, the Sudbury Neutrino Observatory has provided strong evidence that neutrinos do change flavour as they propagate from the core of the Sun [105], independently of solar model flux predictions. SNO is able to detect neutrinos through three different reactions: the charged-current process $\nu_{e} d \rightarrow e^{-} p p$ which is only sensitive to $\nu_{e}$, the neutral current transition $\nu_{x} d \rightarrow \nu_{x} p n$ which has equal probability for all active neutrino flavours, and the elastic scattering $\nu_{x} e^{-} \rightarrow \nu_{x} e^{-}$which is also sensitive to $\nu_{\mu}$ and $\nu_{\tau}$, although the corresponding cross section is a factor 6.48 smaller than the $\nu_{e}$ one. The measured neutrino fluxes, shown in Fig. 34, demonstrate the existence of a non- $\nu_{e}$ component in the solar neutrino flux at the $5.3 \sigma$ level. The SNO results are in good agreement with the Super-Kamiokande solar measurements [108] and have been further reinforced with the more recent KamLAND data, showing that $\bar{\nu}_{e}$ from nuclear reactors disappear over distances of about $180 \mathrm{Km}$ [109], and the Borexino measurement of the monochromatic $0.862 \mathrm{MeV}^{7} \mathrm{Be}$ solar neutrino flux [110].

Another evidence of oscillations has been obtained from atmospheric neutrinos. The known discrepancy between the experimental observations and the predicted ratio of muon to electron neutrinos has become much stronger with the high precision and large statistics of Super-Kamiokande [111]. The atmospheric anomaly appears to originate in a reduction of the $\nu_{\mu}$ flux, and the data strongly favours the $\nu_{\mu} \rightarrow \nu_{\tau}$ hypothesis. Super-Kamiokande has reported statistical evidence of $\nu_{\tau}$ appearance at the $2.4 \sigma$ level [111]. This result has been confirmed by K2K [112] and MINOS [113], observing the disappearance of accelerator $\nu_{\mu}$ 's (and $\bar{\nu}_{\mu}$ 's) at distances of 250 and $735 \mathrm{Km}$, respectively. The direct detection of the produced $\nu_{\tau}$ is the main goal of the ongoing CERN to Gran Sasso neutrino program. The observation of a first $\nu_{\tau}$ candidate event has been recently announced by the OPERA Collaboration [114].

The angle $\theta_{13}$ is strongly constrained by the CHOOZ reactor experiment [115] and by MINOS $\nu_{e}$ appearance searches [113]. More recently, the T2K collaboration has announced [116] evidence for $\nu_{\mu} \rightarrow \nu_{e}$ appearance with a statistical significance of $2.5 \sigma$ (6 events are observed, while the expected number with $\sin ^{2}\left(2 \theta_{13}\right)=0$ is $\left.1.5 \pm 0.3\right)$. This has been confirmed by DOUBLE CHOOZ reactor data, indicating $\bar{\nu}_{e}$ disappearance consistent with neutrino oscillations [117].

Thus, we have now clear experimental evidence that neutrinos are massive particles and there is mixing in the lepton sector. Figures 35, 36 and 37 show the present information on neutrino oscillations, from solar, atmospheric, accelerator and reactor neutrino data. In the limit $\theta_{13}=0$, solar and atmospheric 

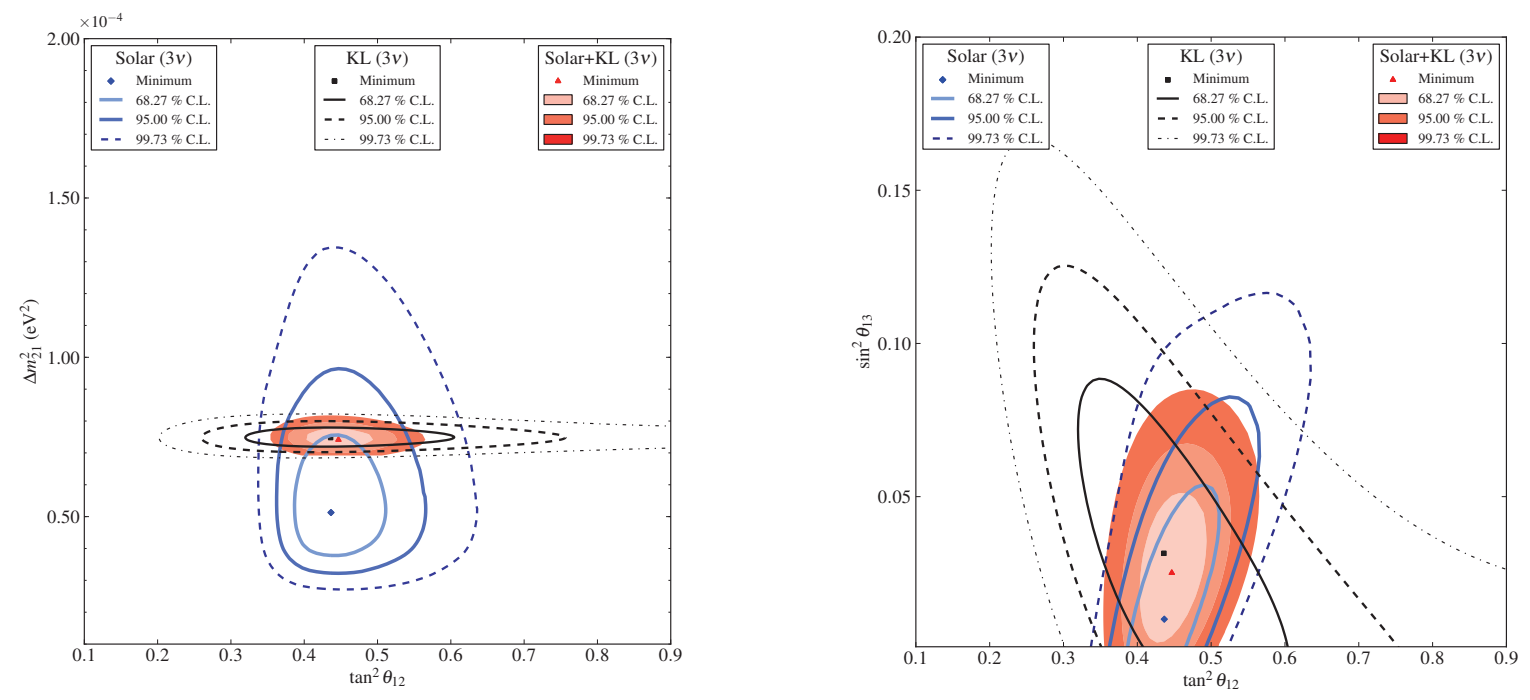

Fig. 35: Allowed regions for three-flavour neutrino oscillations, using both solar neutrino and KamLAND (KL) results, assuming $\mathcal{C P} \mathcal{T}$ symmetry [105].

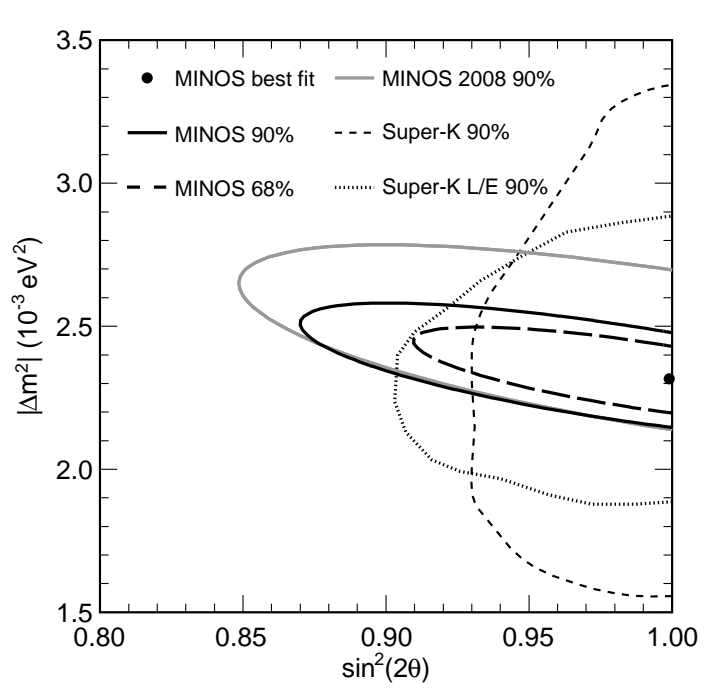

Fig. 36: MINOS allowed regions for $\nu_{\mu}$ disappearance oscillations, compared with previous results [113].

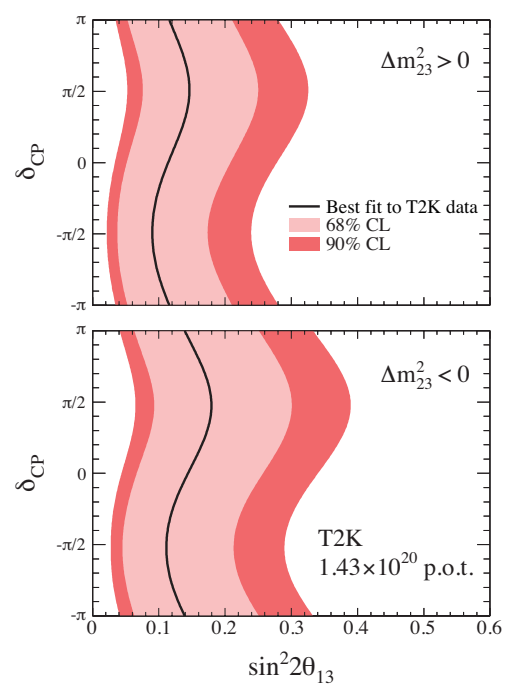

Fig. 37: Constraints on $\sin ^{2}\left(2 \theta_{13}\right)$ versus the $\mathcal{C P}$ phase $\delta_{C P} \equiv \delta_{13}$ from T2K data [116].

neutrino oscillations decouple because $\Delta m_{\odot}^{2} \ll \Delta m_{\text {atm }}^{2}$. Thus, $\Delta m_{21}^{2}, \theta_{12}$ and $\theta_{13}$ are constrained by solar data, while atmospheric experiments constrain $\Delta m_{31}^{2}, \theta_{23}$ and $\theta_{13}$. A global analysis, combining the full set of data, leads to the following preferred ranges for the oscillation parameters [118]:

$$
\begin{gathered}
\Delta m_{21}^{2}=\left(7.59_{-0.18}^{+0.20}\right) \cdot 10^{-5} \mathrm{eV}^{2}, \quad \Delta m_{31}^{2}=\left\{\begin{array}{c}
(2.45 \pm 0.09) \cdot 10^{-3} \mathrm{eV}^{2} \\
-\left(2.34_{-0.09}^{+0.10}\right) \cdot 10^{-3} \mathrm{eV}^{2}
\end{array}, \quad \sin ^{2} \theta_{23}=\left\{\begin{array}{l}
0.51 \pm 0.06 \\
0.52 \pm 0.06
\end{array}, \quad \sin ^{2} \theta_{13}=\left\{\begin{array}{l}
0.013_{-0.005}^{+0.007} \\
0.016_{-0.006}^{+0.008}
\end{array},\right.\right.\right. \\
\sin ^{2} \theta_{12}=0.312_{-0.015}^{+0.017},
\end{gathered}
$$

where $\Delta m_{i j}^{2} \equiv m_{i}^{2}-m_{j}^{2}$ are the mass squared differences between the neutrino mass eigenstates $\nu_{i, j}$, $\theta_{i j}$ the corresponding angles in the standard three-flavour parametrization of the neutrino mixing matrix 
$\mathbf{V}_{L}$ [9], analogous to the CKM matrix in Eq. (118), and the upper (lower) rows correspond to normal (inverted) neutrino mass hierarchy. The combination of the DOUBLE CHOOZ (not yet included in the global fit), T2K and MINOS measurements leads to $0.003<\sin ^{2}\left(2 \theta_{13}\right)<0.219$ at the $3 \sigma$ level [117].

Non-zero neutrino masses constitute a clear indication of new physics beyond the SM. Righthanded neutrinos are an obvious possibility to incorporate Dirac neutrino masses. However, the $\nu_{i R}$ fields would be $S U(3)_{C} \otimes S U(2)_{L} \otimes U(1)_{Y}$ singlets, without any SM interaction. If such objects do exist, it would seem natural to expect that they are able to communicate with the rest of the world through some still unknown dynamics. Moreover, the SM gauge symmetry would allow for a right-handed Majorana neutrino mass term,

$$
\mathcal{L}_{M}=-\frac{1}{2} \overline{\nu_{i R}^{c}} M_{i j} \nu_{j R}+\text { h.c. },
$$

where $\nu_{i R}^{c} \equiv \mathcal{C} \bar{\nu}_{i R}^{T}$ denotes the charge-conjugated field. The Majorana mass matrix $M_{i j}$ could have an arbitrary size, because it is not related to the ordinary Higgs mechanism. Since both fields $\nu_{i R}$ and $\overline{\nu_{i R}^{c}}$ absorb $\nu$ and create $\bar{\nu}$, the Majorana mass term mixes neutrinos and anti-neutrinos, violating lepton number by two units. Clearly, new physics is called for.

Adopting a more general effective field theory language, without any assumption about the existence of right-handed neutrinos or any other new particles, one can write the most general $S U(3)_{C} \otimes$ $S U(2)_{L} \otimes U(1)_{Y}$ invariant Lagrangian, in terms of the known low-energy fields (left-handed neutrinos only). The SM is the unique answer with dimension four. The first contributions from new physics appear through dimension- 5 operators, and have also a unique form which violates lepton number by two units [119]:

$$
\Delta \mathcal{L}=-\frac{c_{i j}}{\Lambda} \bar{L}_{i} \tilde{\phi} \tilde{\phi}^{t} L_{j}^{c}+\text { h.c. },
$$

where $L_{i}$ denotes the $i$-flavoured $S U(2)_{L}$ lepton doublet, $\tilde{\phi} \equiv i \tau_{2} \phi^{*}$ and $L_{i}^{c} \equiv \mathcal{C} \bar{L}_{i}^{T}$. Similar operators with quark fields are forbidden, due to their different hypercharges, while higher-dimension operators would be suppressed by higher powers of the new-physics scale $\Lambda$. After SSB, $\left\langle\phi^{(0)}\right\rangle=v / \sqrt{2}, \Delta \mathcal{L}$ generates a Majorana mass term for the left-handed neutrinos, with $M_{i j}=c_{i j} v^{2} / \Lambda$. Thus, Majorana neutrino masses should be expected on general symmetry grounds. Taking $m_{\nu} \gtrsim 0.05 \mathrm{eV}$, as suggested by atmospheric neutrino data, one gets $\Lambda / c_{i j} \lesssim 10^{15} \mathrm{GeV}$, amazingly close to the expected scale of Gran Unification.

With non-zero neutrino masses, the leptonic charged-current interactions involve a flavour mixing matrix $\mathbf{V}_{L}$. The data on neutrino oscillations imply that all elements of $\mathbf{V}_{L}$ are large, except for $\left(\mathbf{V}_{L}\right)_{e 3}<0.17$ (90\% C.L.); therefore the mixing among leptons appears to be very different from the one in the quark sector. The number of relevant phases characterizing the matrix $\mathbf{V}_{L}$ depends on the Dirac or Majorana nature of neutrinos, because if one rotates a Majorana neutrino by a phase, this phase will appear in its mass term which will no longer be real. With only three Majorana (Dirac) neutrinos, the $3 \times 3$ matrix $\mathbf{V}_{L}$ involves six (four) independent parameters: three mixing angles and three (one) phases.

The smallness of neutrino masses implies a strong suppression of neutrinoless lepton-flavourviolating processes, which can be avoided in models with other sources of lepton-flavour violation, not related to $m_{\nu_{i}}$. Table 5 shows some representative published limits on lepton-flavour-violating decays. The B-Factory data are pushing the experimental limits on neutrinoless $\tau$ decays to the $10^{-8}$ level, increasing in a drastic way the sensitivity to new physics scales. Future experiments could push further some limits to the $10^{-9}$ level, allowing to explore interesting and totally unknown phenomena. Complementary information will be provided by the MEG experiment, which is now searching for $\mu^{+} \rightarrow e^{+} \gamma$ events with a sensitivity of $10^{-13}$ [64]. There are also ongoing projects at Fermilab [122] and J-PARC [123] aiming to study $\mu \rightarrow e$ conversions in muonic atoms at the $10^{-16}$ level, and new proposals to reach sensitivities around $10^{-18}$ are being considered [124].

\footnotetext{
${ }^{4}$ This relation generalizes the well-known see-saw mechanism $\left(m_{\nu_{L}} \sim m^{2} / \Lambda\right)[120,121]$.
} 
Table 5: Some published limits (90\% C.L.) on lepton-flavour-violating decays [63, 66, 125].

\begin{tabular}{lll}
\hline \hline $\operatorname{Br}\left(\mu^{-} \rightarrow e^{-} \gamma\right)<2.4 \cdot 10^{-12}$ & $\operatorname{Br}\left(\mu^{-} \rightarrow e^{-} 2 \gamma\right)<7.2 \cdot 10^{-11}$ & $\operatorname{Br}\left(\mu^{-} \rightarrow e^{-} e^{-} e^{+}\right)<1.0 \cdot 10^{-12}$ \\
$\operatorname{Br}\left(\tau^{-} \rightarrow \mu^{-} \gamma\right)<4.4 \cdot 10^{-8}$ & $\operatorname{Br}\left(\tau^{-} \rightarrow e^{-} \gamma\right)<3.3 \cdot 10^{-8}$ & $\operatorname{Br}\left(\tau^{-} \rightarrow e^{-} e^{-} \mu^{+}\right)<1.5 \cdot 10^{-8}$ \\
$\operatorname{Br}\left(\tau^{-} \rightarrow e^{-} K_{S}\right)<2.6 \cdot 10^{-8}$ & $\operatorname{Br}\left(\tau^{-} \rightarrow \mu^{-} K_{S}\right)<2.3 \cdot 10^{-8}$ & $\operatorname{Br}\left(\tau^{-} \rightarrow \mu^{+} \pi^{-} \pi^{-}\right)<3.7 \cdot 10^{-8}$ \\
$\operatorname{Br}\left(\tau^{-} \rightarrow \Lambda \pi^{-}\right)<7.2 \cdot 10^{-8}$ & $\operatorname{Br}\left(\tau^{-} \rightarrow e^{-} \pi^{0}\right)<8.0 \cdot 10^{-8}$ & $\operatorname{Br}\left(\tau^{-} \rightarrow e^{-} \pi^{+} \pi^{-}\right)<4.4 \cdot 10^{-8}$ \\
$\operatorname{Br}\left(\tau^{-} \rightarrow \mu^{-} \rho^{0}\right)<2.6 \cdot 10^{-8}$ & $\operatorname{Br}\left(\tau^{-} \rightarrow \mu^{-} \eta\right)<6.5 \cdot 10^{-8}$ & $\operatorname{Br}\left(\tau^{-} \rightarrow \mu^{-} e^{+} \mu^{-}\right)<1.7 \cdot 10^{-8}$ \\
\hline \hline
\end{tabular}

At present, we still ignore whether neutrinos are Dirac or Majorana fermions. Another important question to be addressed in the future concerns the possibility of leptonic $\mathrm{CP}$ violation and its relevance for explaining the baryon asymmetry of our Universe through leptogenesis.

\section{Summary}

The SM provides a beautiful theoretical framework which is able to accommodate all our present knowledge on electroweak and strong interactions. It is able to explain any single experimental fact and, in some cases, it has successfully passed very precise tests at the $0.1 \%$ to $1 \%$ level. In spite of this impressive phenomenological success, the SM leaves too many unanswered questions to be considered as a complete description of the fundamental forces. We do not understand yet why fermions are replicated in three (and only three) nearly identical copies. Why the pattern of masses and mixings is what it is? Are the masses the only difference among the three families? What is the origin of the SM flavour structure? Which dynamics is responsible for the observed $\mathcal{C P}$ violation?

In the gauge and scalar sectors, the SM Lagrangian contains only four parameters: $g, g^{\prime}, \mu^{2}$ and $h$. We can trade them by $\alpha, M_{Z}, G_{F}$ and $M_{H}$; this has the advantage of using the three most precise experimental determinations to fix the interaction. In any case, one describes a lot of physics with only four inputs. In the fermionic flavour sector, however, the situation is very different. With $N_{G}=3$, we have 13 additional free parameters in the minimal SM: 9 fermion masses, 3 quark mixing angles and 1 phase. Taking into account non-zero neutrino masses, we have three more mass parameters plus the leptonic mixings: three angles and one phase (three phases) for Dirac (or Majorana) neutrinos.

Clearly, this is not very satisfactory. The source of this proliferation of parameters is the set of unknown Yukawa couplings in Eq. (110). The origin of masses and mixings, together with the reason for the existing family replication, constitute at present the main open problem in electroweak physics. The generation of fermion masses is deeply related with the mechanism responsible for the electroweak SSB. Thus, the origin of these parameters lies in the most obscure part of the SM Lagrangian: the scalar sector. The dynamics of flavour appears to be 'terra incognita' which deserves a careful investigation.

The SM incorporates a mechanism to generate $\mathcal{C P}$ violation, through the single phase naturally occurring in the CKM matrix. This mechanism, deeply rooted into the unitarity structure of $\mathbf{V}$, implies very specific requirements for $\mathcal{C P}$ violation to show up. The CKM matrix has been thoroughly investigated in dedicated experiments and a large number of $\mathcal{C P}$-violating processes have been studied in detail. At present, all quark flavour data seem to fit into the SM framework, confirming that the fermion mass matrices are the dominant source of flavour-mixing phenomena. However, the SM is unable to explain the matter-antimatter asymmetry of our Universe. A fundamental explanation of the flavour dynamics and $\mathcal{C P}$-violating phenomena is still lacking.

The first hints of new physics beyond the SM have emerged recently, with convincing evidence of neutrino oscillations showing that $\nu_{i} \rightarrow \nu_{j}(i \neq j)$ transitions do occur. The existence of lepton-flavour violation opens a very interesting window to unknown phenomena. In fact, the smallness of neutrino masses suggests new physics at very high energies, close to the expected scale of Gran Unification. 
The Higgs particle is the main missing block of the SM. The LHC has already excluded a broad range of Higgs masses, narrowing down the SM Higgs hunting to the low-mass region between 115.5 and $127 \mathrm{GeV}(95 \% \mathrm{CL})[59,60]$. This is precisely the range of masses preferred by precision electroweak tests. In the next months the LHC should find out whether such scalar field indeed exists. The discovery of a neutral boson in this mass range would provide a spectacular confirmation of the SM framework.

If the Higgs boson does not show up soon, we should look for alternative mechanisms of mass generation, satisfying the many experimental constraints which the SM has successfully fulfilled so far. The easiest perturbative way would be enlarging the scalar sector with additional Higgs bosons; new scalar doublets could easily comply with the present phenomenological requirements. Another interesting possibility would be dynamical electroweak symmetry breaking, either without Higgs bosons, as happens in QCD with chiral symmetry, or with composite scalars. In all cases, flavour phenomena imposes severe restrictions on the possible scenarios for new physics beyond the SM.

Many interesting experimental signals are expected to be seen in the next few years. LHC is already exploring the frontiers of the SM and its possible extensions. Other experiments will probe the dynamics of flavour to a much deeper level of sensitivity, complementing the high-energy searches for new phenomena. Unexpected surprises may well be expected, probably giving hints of new physics at higher scales and offering clues to the problems of mass generation, fermion mixing and family replication.

\section{Acknowledgements}

I want to thank all the students who attended these lectures for their many interesting questions and comments. I would also like to thank the hospitality of the Physics Department of the Technical University of Munich, where these notes have been updated, and the support of the Alexander von Humboldt Foundation. This work has been supported in part by MICINN, Spain (FPA2007-60323 and Consolider-Ingenio 2010 Program CSD2007-00042 -CPAN-) and Generalitat Valenciana (Prometeo/2008/069). 


\section{Appendices}

\section{A Basic Inputs from Quantum Field Theory}

\section{A.1 Wave equations}

The classical Hamiltonian of a non-relativistic free particle is given by $H=\vec{p}^{2} /(2 m)$. In quantum mechanics, energy and momentum correspond to operators acting on the particle wave function. The substitutions $H=i \hbar \frac{\partial}{\partial t}$ and $\vec{p}=-i \hbar \vec{\nabla}$ lead then to the Schrödinger equation:

$$
i \hbar \frac{\partial}{\partial t} \psi(\vec{x}, t)=-\frac{\hbar^{2}}{2 m} \vec{\nabla}^{2} \psi(\vec{x}, t) .
$$

We can write the energy and momentum operators in a relativistic covariant way as $p^{\mu}=i \partial^{\mu} \equiv i \frac{\partial}{\partial x_{\mu}}$, where we have adopted the usual natural units convention $\hbar=c=1$. The relation $E^{2}=\vec{p}^{2}+m^{2}$ determines the Klein-Gordon equation for a relativistic free particle:

$$
\left(\square+m^{2}\right) \phi(x)=0, \quad \square \equiv \partial^{\mu} \partial_{\mu}=\frac{\partial^{2}}{\partial t^{2}}-\vec{\nabla}^{2} .
$$

The Klein-Gordon equation is quadratic on the time derivative because relativity puts the space and time coordinates on an equal footing. Let us investigate whether an equation linear in derivatives could exist. Relativistic covariance and dimensional analysis restrict its possible form to

$$
\left(i \gamma^{\mu} \partial_{\mu}-m\right) \psi(x)=0 .
$$

Since the r.h.s. is identically zero, we can fix the coefficient of the mass term to be -1 ; this just determines the normalization of the four coefficients $\gamma^{\mu}$. Notice that $\gamma^{\mu}$ should transform as a Lorentz fourvector. The solutions of Eq. (A.3) should also satisfy the Klein-Gordon relation of Eq. (A.2). Applying an appropriate differential operator to Eq. (A.3), one can easily obtain the wanted quadratic equation:

$$
-\left(i \gamma^{\nu} \partial_{\nu}+m\right)\left(i \gamma^{\mu} \partial_{\mu}-m\right) \psi(x)=0 \equiv\left(\square+m^{2}\right) \psi(x) .
$$

Terms linear in derivatives cancel identically, while the term with two derivatives reproduces the operator $\square \equiv \partial^{\mu} \partial_{\mu}$ provided the coefficients $\gamma^{\mu}$ satisfy the algebraic relation

$$
\left\{\gamma^{\mu}, \gamma^{\nu}\right\} \equiv \gamma^{\mu} \gamma^{\nu}+\gamma^{\nu} \gamma^{\mu}=2 g^{\mu \nu}
$$

which defines the so-called Dirac algebra. Eq. (A.3) is known as the Dirac equation.

Obviously the components of the four-vector $\gamma^{\mu}$ cannot simply be numbers. The three $2 \times 2$ Pauli matrices satisfy $\left\{\sigma^{i}, \sigma^{j}\right\}=2 \delta^{i j}$, which is very close to the relation A.5. The lowest-dimensional solution to the Dirac algebra is obtained with $D=4$ matrices. An explicit representation is given by:

$$
\gamma^{0}=\left(\begin{array}{cc}
I_{2} & 0 \\
0 & -I_{2}
\end{array}\right), \quad \gamma^{i}=\left(\begin{array}{cc}
0 & \sigma^{i} \\
-\sigma^{i} & 0
\end{array}\right)
$$

Thus, the wave function $\psi(x)$ is a column vector with four components in the Dirac space. The presence of the Pauli matrices strongly suggests that it contains two components of spin $\frac{1}{2}$. A proper physical analysis of its solutions shows that the Dirac equation describes simultaneously a fermion of spin $\frac{1}{2}$ and its own antiparticle [126].

It turns useful to define the following combinations of gamma matrices:

$$
\sigma^{\mu \nu} \equiv \frac{i}{2}\left[\gamma^{\mu}, \gamma^{\nu}\right], \quad \gamma_{5} \equiv \gamma^{5} \equiv i \gamma^{0} \gamma^{1} \gamma^{2} \gamma^{3}=-\frac{i}{4 !} \epsilon_{\mu \nu \rho \sigma} \gamma^{\mu} \gamma^{\nu} \gamma^{\rho} \gamma^{\sigma}
$$


In the explicit representation (A.6),

$$
\sigma^{i j}=\epsilon^{i j k}\left(\begin{array}{cc}
\sigma^{k} & 0 \\
0 & \sigma^{k}
\end{array}\right), \quad \sigma^{0 i}=i\left(\begin{array}{cc}
0 & \sigma^{i} \\
\sigma^{i} & 0
\end{array}\right), \quad \gamma_{5}=\left(\begin{array}{cc}
0 & I_{2} \\
I_{2} & 0
\end{array}\right) .
$$

The matrix $\sigma^{i j}$ is then related to the spin operator. Some important properties are:

$$
\gamma^{0} \gamma^{\mu} \gamma^{0}=\gamma^{\mu \dagger}, \quad \gamma^{0} \gamma_{5} \gamma^{0}=-\gamma_{5}^{\dagger}=-\gamma_{5}, \quad\left\{\gamma_{5}, \gamma^{\mu}\right\}=0, \quad\left(\gamma_{5}\right)^{2}=I_{4} .
$$

Specially relevant for weak interactions are the chirality projectors $\left(P_{L}+P_{R}=1\right)$

$$
P_{L} \equiv \frac{1-\gamma_{5}}{2}, \quad P_{R} \equiv \frac{1+\gamma_{5}}{2}, \quad P_{R}^{2}=P_{R}, \quad P_{L}^{2}=P_{L}, \quad P_{L} P_{R}=P_{R} P_{L}=0,
$$

which allow to decompose the Dirac spinor in its left-handed and right-handed chirality parts:

$$
\psi(x)=\left[P_{L}+P_{R}\right] \psi(x) \equiv \psi_{L}(x)+\psi_{R}(x) .
$$

In the massless limit, the chiralities correspond to the fermion helicities.

\section{A.2 Lagrangian formalism}

The Lagrangian formulation of a physical system provides a compact dynamical description and makes it easier to discuss the underlying symmetries. Like in classical mechanics, the dynamics is encoded in the action

$$
S=\int d^{4} x \quad \mathcal{L}\left[\phi_{i}(x), \partial_{\mu} \phi_{i}(x)\right] .
$$

The integral over the four space-time coordinates preserves relativistic invariance. The Lagrangian density $\mathcal{L}$ is a Lorentz-invariant functional of the fields $\phi_{i}(x)$ and their derivatives. The space integral $L=\int d^{3} x \mathcal{L}$ would correspond to the usual non-relativistic Lagrangian.

The principle of stationary action requires the variation $\delta S$ of the action to be zero under small fluctuations $\delta \phi_{i}$ of the fields. Assuming that the variations $\delta \phi_{i}$ are differentiable and vanish outside some bounded region of space-time (which allows an integration by parts), the condition $\delta S=0$ determines the Euler-Lagrange equations of motion for the fields:

$$
\frac{\partial \mathcal{L}}{\partial \phi_{i}}-\partial^{\mu}\left(\frac{\partial \mathcal{L}}{\partial\left(\partial^{\mu} \phi_{i}\right)}\right)=0
$$

One can easily find appropriate Lagrangians to generate the Klein-Gordon and Dirac equations. They should be quadratic on the fields and Lorentz invariant, which determines their possible form up to irrelevant total derivatives. The Lagrangian

$$
\mathcal{L}=\partial^{\mu} \phi^{*} \partial_{\mu} \phi-m^{2} \phi^{*} \phi
$$

describes a complex scalar field without interactions. Both the field $\phi(x)$ and its complex conjugate $\phi^{*}(x)$ satisfy the Klein-Gordon equation; thus, $\phi(x)$ describes a particle of mass $m$ without spin and its antiparticle. Particles which are their own antiparticles (i.e., with no internal charges) have only one degree of freedom and are described through a real scalar field. The appropriate Klein-Gordon Lagrangian is then

$$
\mathcal{L}=\frac{1}{2} \partial^{\mu} \phi \partial_{\mu} \phi-\frac{1}{2} m^{2} \phi^{2} .
$$

The Dirac equation can be derived from the Lagrangian density

$$
\mathcal{L}=\bar{\psi}\left(i \gamma^{\mu} \partial_{\mu}-m\right) \psi .
$$


The adjoint spinor $\bar{\psi}(x)=\psi^{\dagger}(x) \gamma^{0}$ closes the Dirac indices. The matrix $\gamma^{0}$ is included to guarantee the proper behaviour under Lorentz transformations: $\bar{\psi} \psi$ is a Lorentz scalar, while $\bar{\psi} \gamma^{\mu} \psi$ transforms as a four-vector [126]. Therefore, $\mathcal{L}$ is Lorentz invariant as it should.

Using the decomposition A.11) of the Dirac field in its two chiral components, the fermionic Lagrangian adopts the form:

$$
\mathcal{L}=\bar{\psi}_{L} i \gamma^{\mu} \partial_{\mu} \psi_{L}+\bar{\psi}_{R} i \gamma^{\mu} \partial_{\mu} \psi_{R}-m\left(\bar{\psi}_{L} \psi_{R}+\bar{\psi}_{R} \psi_{L}\right)
$$

Thus, the two chiralities decouple if the fermion is massless.

\section{A.3 Symmetries and conservation laws}

Let us assume that the Lagrangian of a physical system is invariant under some set of continuous transformations

$$
\phi_{i}(x) \rightarrow \phi_{i}^{\prime}(x)=\phi_{i}(x)+\epsilon \delta_{\epsilon} \phi_{i}(x)+O\left(\epsilon^{2}\right),
$$

i.e., $\mathcal{L}\left[\phi_{i}(x), \partial_{\mu} \phi_{i}(x)\right]=\mathcal{L}\left[\phi_{i}^{\prime}(x), \partial_{\mu} \phi_{i}^{\prime}(x)\right]$. One finds then that

$$
\delta_{\epsilon} \mathcal{L}=0=\sum_{i}\left\{\left[\frac{\partial \mathcal{L}}{\partial \phi_{i}}-\partial^{\mu}\left(\frac{\partial \mathcal{L}}{\partial\left(\partial^{\mu} \phi_{i}\right)}\right)\right] \delta_{\epsilon} \phi_{i}+\partial^{\mu}\left[\frac{\partial \mathcal{L}}{\partial\left(\partial^{\mu} \phi_{i}\right)} \delta_{\epsilon} \phi_{i}\right]\right\} .
$$

If the fields satisfy the Euler-Lagrange equations of motion (A.13), the first term is identically zero; therefore the system has a conserved current:

$$
J_{\mu} \equiv \sum_{i} \frac{\partial \mathcal{L}}{\partial\left(\partial^{\mu} \phi_{i}\right)} \delta_{\epsilon} \phi_{i}, \quad \partial^{\mu} J_{\mu}=0 .
$$

This allows us to define a conserved charge

$$
\mathcal{Q} \equiv \int d^{3} x J^{0}
$$

The condition $\partial^{\mu} J_{\mu}=0$ guarantees that $\frac{d \mathcal{Q}}{d t}=0$, i.e., that $\mathcal{Q}$ is a constant of motion.

This result, known as Noether's theorem, can be easily extended to general transformations involving also the space-time coordinates. For every continuous symmetry transformation which leaves the action invariant, there is a corresponding divergenceless Noether's current and, therefore, a conserved charge. The selection rules observed in Nature, where there exist several conserved quantities (energy, momentum, angular momentum, electric charge, etc.), correspond to dynamical symmetries of the Lagrangian.

\section{A.4 Classical electrodynamics}

The well-known Maxwell equations,

$$
\begin{array}{ll}
\vec{\nabla} \cdot \vec{B}=0, & \vec{\nabla} \times \vec{E}+\frac{\partial \vec{B}}{\partial t}=0, \\
\vec{\nabla} \cdot \vec{E}=\rho, & \vec{\nabla} \times \vec{B}-\frac{\partial \vec{E}}{\partial t}=\vec{J},
\end{array}
$$

summarize a large amount of experimental and theoretical work and provide a unified description of the electric and magnetic forces. The first two equations in (A.22) are easily solved, writing the electromagnetic fields in terms of potentials:

$$
\vec{E}=-\vec{\nabla} V-\frac{\partial \vec{A}}{\partial t}, \quad \vec{B}=\vec{\nabla} \times \vec{A}
$$


It is very useful to rewrite these equations in a Lorentz covariant notation. The charge density $\rho$ and the electromagnetic current $\vec{J}$ transform as a four-vector $J^{\mu} \equiv(\rho, \vec{J})$. The same is true for the potentials which combine into $A^{\mu} \equiv(V, \vec{A})$. The relations (A.24) between the potentials and the fields then take a very simple form, which defines the field strength tensor:

$$
F^{\mu \nu} \equiv \partial^{\mu} A^{\nu}-\partial^{\nu} A^{\mu}=\left(\begin{array}{cccc}
0 & -E_{1} & -E_{2} & -E_{3} \\
E_{1} & 0 & -B_{3} & B_{2} \\
E_{2} & B_{3} & 0 & -B_{1} \\
E_{3} & -B_{2} & B_{1} & 0
\end{array}\right), \quad \tilde{F}^{\mu \nu} \equiv \frac{1}{2} \epsilon^{\mu \nu \rho \sigma} F_{\rho \sigma} .
$$

In terms of the tensor $F^{\mu \nu}$, the covariant form of the Maxwell equations turns out to be very transparent:

$$
\partial_{\mu} \tilde{F}^{\mu \nu}=0, \quad \partial_{\mu} F^{\mu \nu}=J^{\nu} .
$$

The electromagnetic dynamics is clearly a relativistic phenomenon, but Lorentz invariance was not very explicit in the original formulation of Eqs. A.22 and (A.23). Once a covariant formulation is adopted, the equations become much simpler. The conservation of the electromagnetic current appears now as a natural compatibility condition:

$$
\partial_{\nu} J^{\nu}=\partial_{\nu} \partial_{\mu} F^{\mu \nu}=0 .
$$

In terms of potentials, $\partial_{\mu} \tilde{F}^{\mu \nu}$ is identically zero while $\partial_{\mu} F^{\mu \nu}=J^{\nu}$ adopts the form:

$$
\square A^{\nu}-\partial^{\nu}\left(\partial_{\mu} A^{\mu}\right)=J^{\nu} \text {. }
$$

The same dynamics can be described by many different electromagnetic four-potentials, which give the same field strength tensor $F^{\mu \nu}$. Thus, the Maxwell equations are invariant under gauge transformations:

$$
A^{\mu} \longrightarrow A^{\prime \mu}=A^{\mu}+\partial^{\mu} \Lambda .
$$

Taking the Lorentz gauge $\partial_{\mu} A^{\mu}=0$, Eq. (A.28) simplifies to

$$
\square A^{\nu}=J^{\nu} .
$$

In the absence of an external current, i.e., with $J^{\mu}=0$, the four components of $A^{\mu}$ satisfy then a Klein-Gordon equation with $m=0$. The photon is therefore a massless particle.

The Lorentz condition $\partial_{\mu} A^{\mu}=0$ still allows for a residual gauge invariance under transformations of the type (A.29), with the restriction $\square \Lambda=0$. Thus, we can impose a second constraint on the electromagnetic field $A^{\mu}$, without changing $F^{\mu \nu}$. Since $A^{\mu}$ contains four fields $(\mu=0,1,2,3)$ and there are two arbitrary constraints, the number of physical degrees of freedom is just two. Therefore, the photon has two different physical polarizations

\section{B $\mathbf{S U}(\mathbf{N})$ Algebra}

$S U(N)$ is the group of $N \times N$ unitary matrices, $U U^{\dagger}=U^{\dagger} U=1$, with $\operatorname{det} U=1$. Any $S U(N)$ matrix can be written in the form

$$
U=\exp \left\{i T^{a} \theta_{a}\right\}, \quad a=1,2, \ldots, N^{2}-1,
$$

with $T^{a}=\lambda^{a} / 2$ Hermitian, traceless matrices. Their commutation relations

$$
\left[T^{a}, T^{b}\right]=i f^{a b c} T^{c}
$$

define the $S U(N)$ algebra. The $N \times N$ matrices $\lambda^{a} / 2$ generate the fundamental representation of the $S U(N)$ algebra. The basis of generators $\lambda^{a} / 2$ can be chosen so that the structure constants $f^{a b c}$ are real and totally antisymmetric. 
For $N=2, \lambda^{a}$ are the usual Pauli matrices,

$$
\sigma_{1}=\left(\begin{array}{cc}
0 & 1 \\
1 & 0
\end{array}\right), \quad \sigma_{2}=\left(\begin{array}{cc}
0 & -i \\
i & 0
\end{array}\right), \quad \sigma_{3}=\left(\begin{array}{cc}
1 & 0 \\
0 & -1
\end{array}\right)
$$

which satisfy the commutation relation

$$
\left[\sigma_{i}, \sigma_{j}\right]=2 i \epsilon_{i j k} \sigma_{k}
$$

Other useful properties are: $\left\{\sigma_{i}, \sigma_{j}\right\}=2 \delta_{i j}$ and $\operatorname{Tr}\left(\sigma_{i} \sigma_{j}\right)=2 \delta_{i j}$.

For $N=3$, the fundamental representation corresponds to the eight Gell-Mann matrices:

$$
\begin{aligned}
& \lambda^{1}=\left(\begin{array}{lll}
0 & 1 & 0 \\
1 & 0 & 0 \\
0 & 0 & 0
\end{array}\right), \quad \lambda^{2}=\left(\begin{array}{ccc}
0 & -i & 0 \\
i & 0 & 0 \\
0 & 0 & 0
\end{array}\right), \quad \lambda^{3}=\left(\begin{array}{ccc}
1 & 0 & 0 \\
0 & -1 & 0 \\
0 & 0 & 0
\end{array}\right), \quad \lambda^{4}=\left(\begin{array}{ccc}
0 & 0 & 1 \\
0 & 0 & 0 \\
1 & 0 & 0
\end{array}\right), \\
& \lambda^{5}=\left(\begin{array}{ccc}
0 & 0 & -i \\
0 & 0 & 0 \\
i & 0 & 0
\end{array}\right), \lambda^{6}=\left(\begin{array}{ccc}
0 & 0 & 0 \\
0 & 0 & 1 \\
0 & 1 & 0
\end{array}\right), \quad \lambda^{7}=\left(\begin{array}{ccc}
0 & 0 & 0 \\
0 & 0 & -i \\
0 & i & 0
\end{array}\right), \quad \lambda^{8}=\frac{1}{\sqrt{3}}\left(\begin{array}{ccc}
1 & 0 & 0 \\
0 & 1 & 0 \\
0 & 0 & -2
\end{array}\right) \text {. }
\end{aligned}
$$

They satisfy the anticommutation relation

$$
\left\{\lambda^{a}, \lambda^{b}\right\}=\frac{4}{N} \delta^{a b} I_{N}+2 d^{a b c} \lambda^{c},
$$

where $I_{N}$ denotes the $N$-dimensional unit matrix and the constants $d^{a b c}$ are totally symmetric in the three indices.

For $S U(3)$, the only non-zero (up to permutations) $f^{a b c}$ and $d^{a b c}$ constants are

$$
\begin{aligned}
& \frac{1}{2} f^{123}=f^{147}=-f^{156}=f^{246}=f^{257}=f^{345}=-f^{367}=\frac{1}{\sqrt{3}} f^{458}=\frac{1}{\sqrt{3}} f^{678}=\frac{1}{2}, \\
& d^{146}=d^{157}=-d^{247}=d^{256}=d^{344}=d^{355}=-d^{366}=-d^{377}=\frac{1}{2} \\
& d^{118}=d^{228}=d^{338}=-2 d^{448}=-2 d^{558}=-2 d^{668}=-2 d^{778}=-d^{888}=\frac{1}{\sqrt{3}} .
\end{aligned}
$$

The adjoint representation of the $S U(N)$ group is given by the $\left(N^{2}-1\right) \times\left(N^{2}-1\right)$ matrices $\left(T_{A}^{a}\right)_{b c} \equiv-i f^{a b c}$, which satisfy the commutation relations (B.2). The following equalities

$$
\begin{array}{cl}
\operatorname{Tr}\left(\lambda^{a} \lambda^{b}\right)=4 T_{F} \delta_{a b}, & T_{F}=\frac{1}{2}, \\
\left(\lambda^{a} \lambda^{a}\right)_{\alpha \beta}=4 C_{F} \delta_{\alpha \beta}, & C_{F}=\frac{N^{2}-1}{2 N}, \\
\operatorname{Tr}\left(T_{A}^{a} T_{A}^{b}\right)=f^{a c d} f^{b c d}=C_{A} \delta_{a b}, & C_{A}=N,
\end{array}
$$

define the $S U(N)$ invariants $T_{F}, C_{F}$ and $C_{A}$. Other useful properties are:

$$
\begin{array}{cc}
\left(\lambda^{a}\right)_{\alpha \beta}\left(\lambda^{a}\right)_{\gamma \delta}=2 \delta_{\alpha \delta} \delta_{\beta \gamma}-\frac{2}{N} \delta_{\alpha \beta} \delta_{\gamma \delta}, & \operatorname{Tr}\left(\lambda^{a} \lambda^{b} \lambda^{c}\right)=2\left(d^{a b c}+i f^{a b c}\right), \\
\operatorname{Tr}\left(T_{A}^{a} T_{A}^{b} T_{A}^{c}\right)=i \frac{N}{2} f^{a b c}, \quad \sum_{b} d^{a b b}=0, & d^{a b c} d^{e b c}=\left(N-\frac{4}{N}\right) \delta_{a e}, \\
f^{a b e} f^{c d e}+f^{a c e} f^{d b e}+f^{a d e} f^{b c e}=0, & f^{a b e} d^{c d e}+f^{a c e} d^{d b e}+f^{a d e} d^{b c e}=0 .
\end{array}
$$




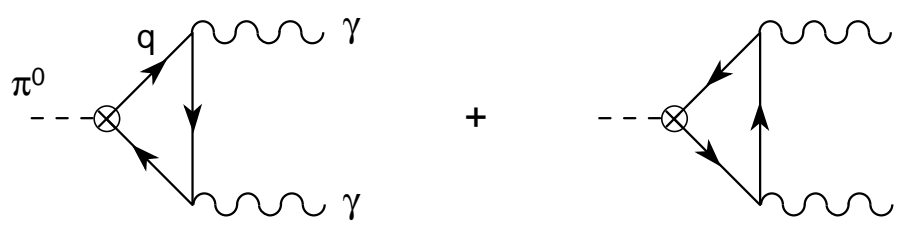

Fig. C.1: Triangular quark loops generating the decay $\pi^{0} \rightarrow \gamma \gamma$.

\section{Anomalies}

Our theoretical framework is based on the local gauge symmetry. However, so far we have only discussed the symmetries of the classical Lagrangian. It happens sometimes that a symmetry of $\mathcal{L}$ gets broken by quantum effects, i.e., it is not a symmetry of the quantized theory; one says then that there is an 'anomaly'. Anomalies appear in those symmetries involving both axial $\left(\bar{\psi} \gamma^{\mu} \gamma_{5} \psi\right)$ and vector $\left(\bar{\psi} \gamma^{\mu} \psi\right)$ currents, and reflect the impossibility of regularizing the quantum theory (the divergent loops) in a way which preserves the chiral (left/right) symmetries.

A priori there is nothing wrong with having an anomaly. In fact, sometimes they are even welcome. A good example is provided by the decay $\pi^{0} \rightarrow \gamma \gamma$. There is a global chiral symmetry of the QCD Lagrangian which forbids this transition; the $\pi^{0}$ should then be a stable particle, in contradiction with the experimental evidence. Fortunately, there is an anomaly generated by a triangular quark loop (Fig. C.1) which couples the axial current $A_{\mu}^{3} \equiv\left(\bar{u} \gamma_{\mu} \gamma_{5} u-\bar{d} \gamma_{\mu} \gamma_{5} d\right)$ to two electromagnetic currents and breaks the conservation of the axial current at the quantum level:

$$
\partial^{\mu} A_{\mu}^{3}=\frac{\alpha}{4 \pi} \epsilon^{\alpha \beta \sigma \rho} F_{\alpha \beta} F_{\sigma \rho}+\mathcal{O}\left(m_{u}+m_{d}\right) .
$$

Since the $\pi^{0}$ couples to $A_{\mu}^{3},\left\langle 0\left|A_{\mu}^{3}\right| \pi^{0}\right\rangle=2 i f_{\pi} p_{\mu}$, the $\pi^{0} \rightarrow \gamma \gamma$ decay does finally occur, with a predicted rate

$$
\Gamma\left(\pi^{0} \rightarrow \gamma \gamma\right)=\left(\frac{N_{C}}{3}\right)^{2} \frac{\alpha^{2} m_{\pi}^{3}}{64 \pi^{3} f_{\pi}^{2}}=7.73 \mathrm{eV},
$$

where $N_{C}=3$ denotes the number of quark colours and the so-called pion decay constant, $f_{\pi}=$ $92.4 \mathrm{MeV}$, is known from the $\pi^{-} \rightarrow \mu^{-} \bar{\nu}_{\mu}$ decay rate (assuming isospin symmetry). The agreement with the measured value, $\Gamma=7.7 \pm 0.5 \mathrm{eV}$ [9], is excellent.

Anomalies are, however, very dangerous in the case of local gauge symmetries, because they destroy the renormalizability of the quantum field theory. Since the $S U(2)_{L} \otimes U(1)_{Y}$ model is chiral (i.e., it distinguishes left from right), anomalies are clearly present. The gauge bosons couple to vector and axial-vector currents; we can then draw triangular diagrams with three arbitrary gauge bosons $\left(W^{ \pm}\right.$, $Z, \gamma)$ in the external legs. Any such diagram involving one axial and two vector currents generates a breaking of the gauge symmetry. Thus, our nice model looks meaningless at the quantum level.

We have still one way out. What matters is not the value of a single Feynman diagram, but the sum of all possible contributions. The anomaly generated by the sum of all triangular diagrams connecting the three gauge bosons $G_{a}, G_{b}$ and $G_{c}$ is proportional to

$$
\mathcal{A}^{a b c}=\operatorname{Tr}\left(\left\{T^{a}, T^{b}\right\} T^{c}\right)_{L}-\operatorname{Tr}\left(\left\{T^{a}, T^{b}\right\} T^{c}\right)_{R},
$$

where the traces sum over all possible left- and right-handed fermions, respectively, running along the internal lines of the triangle. The matrices $T^{a}$ are the generators associated with the corresponding gauge bosons; in our case, $T^{a}=\sigma_{a} / 2, Y$.

In order to preserve the gauge symmetry, one needs a cancellation of all anomalous contributions, i.e., $\mathcal{A}^{a b c}=0$. Since $\operatorname{Tr}\left(\sigma_{k}\right)=0$, we have an automatic cancellation in two combinations of generators: $\operatorname{Tr}\left(\left\{\sigma_{i}, \sigma_{j}\right\} \sigma_{k}\right)=2 \delta^{i j} \operatorname{Tr}\left(\sigma_{k}\right)=0$ and $\operatorname{Tr}\left(\{Y, Y\} \sigma_{k}\right) \propto \operatorname{Tr}\left(\sigma_{k}\right)=0$. However, the other two 
combinations, $\operatorname{Tr}\left(\left\{\sigma_{i}, \sigma_{j}\right\} Y\right)$ and $\operatorname{Tr}\left(Y^{3}\right)$ turn out to be proportional to $\operatorname{Tr}(Q)$, i.e., to the sum of fermion electric charges:

$$
\sum_{i} Q_{i}=Q_{e}+Q_{\nu}+N_{C}\left(Q_{u}+Q_{d}\right)=-1+\frac{1}{3} N_{C}=0 .
$$

Equation (C.4) conveys a very important message: the gauge symmetry of the $S U(2)_{L} \otimes U(1)_{Y}$ model does not have any quantum anomaly, provided that $N_{C}=3$. Fortunately, this is precisely the right number of colours to understand strong interactions. Thus, at the quantum level, the electroweak model seems to know something about QCD. The complete SM gauge theory based on the group $S U(3)_{C} \otimes$ $S U(2)_{L} \otimes U(1)_{Y}$ is free of anomalies and, therefore, renormalizable. The anomaly cancellation involves one complete generation of leptons and quarks: $\nu, e, u, d$. The SM would not make any sense with only leptons or quarks.

\section{References}

[1] Updated version of the lectures given at the 2004 (San Feliu de Guixols, Spain), 2006 (Aronsborg, Sweden) and 2010 (Raseborg, Finland) European Schools of High-Energy Physics: A. Pich, The Standard Model of Electroweak Interactions, Reports CERN-2006-003 and CERN-2007-005, ed. R. Fleischer, p. 1; arXiv:hep-ph/0502010, arXiv:0705.4264 [hep-ph].

[2] S.L. Glashow, Nucl. Phys. 22 (1961) 579.

[3] S. Weinberg, Phys. Rev. Lett. 19 (1967) 1264.

[4] A. Salam, in Elementary Particle Theory, ed. N. Svartholm (Almquist and Wiksells, Stockholm, 1969), p. 367.

[5] S.L. Glashow, J. Iliopoulos and L. Maiani, Phys. Rev. D2 (1970) 1285.

[6] A. Pich, Aspects of Quantum Chromodynamics, arXiv:hep-ph/0001118.

[7] M.H. Seymour, Quantum Chromodynamics, arXiv:hep-ph/0505192v2 (2010).

[8] D.D. Ryutov, Plasma Phys. Control. Fusion 49 (2007) B429.

[9] K. Nakamura et al., J. Phys. G37 (2010) 075021; http://pdg.lbl.gov.

[10] D. Hanneke, S. Fogwell and G. Gabrielse, Phys. Rev. Lett. 100 (2008) 120801.

[11] Muon $g$ - 2 Collaboration, Phys. Rev. D73 (2006) 072003.

[12] T. Kinoshita and M. Nio, Phys. Rev. D73 (2006) 013003, 053007; D70 (2004) 113001; Phys. Rev. Lett. 90 (2003) 021803.

[13] T. Aoyama et al., Phys. Rev. D84 (2011) 053003; D83 (2011) 053002, 053003; D82 (2010) 113004; D81 (2010) 053009; D78 (2008) 113006, 053005; D77 (2008) 053012; Phys. Rev. Lett. 99 (2007) 110406; Nucl. Phys. B740 (2006) 138; arXiv:1110.2826 [hep-ph].

[14] A.L. Kataev, Phys. Rev. D74 (2006) 073011.

[15] G. Gabrielse et al., Phys. Rev. Lett. 97 (2006) 030802; Erratum-ibid. 99 (2007) 039902.

[16] M. Davier and W.J. Marciano, Annu. Rev. Nucl. Part. Sci. 54 (2004) 115.

[17] J.P. Miller, E. de Rafael and B.L. Roberts, Rept. Prog. Phys. 70 (2007) 795.

[18] F. Jegerlehner and A. Nyffeler, Phys. Rept. 477 (2009) 1.

[19] M. Davier et al., Eur. Phys. J. C71 (2011) 1515.

[20] J. Prades, E. de Rafael and A. Vainshtein, in Lepton Dipole Moments, eds. B.L. Roberts and W.J. Marciano, Advanced Series on Directions in High Energy Physics, Vol. 20, p. 303 (World Scientific, 2011), arXiv:0901.0306 [hep-ph].

[21] H. Fritzsch and M. Gell-Mann, Proc. XVI International Conference on High Energy Physics, eds. J.D. Jackson and A. Roberts (Fermilab, 1972), Vol. 2, p. 135.

[22] H. Fritzsch, M. Gell-Mann and H. Leutwyler, Phys. Lett. B47 (365) 1973. 
[23] ALEPH Collaboration, http://aleph.web.cern.ch/aleph/dali/Z0_examples.html.

[24] A. Pich, The Standard Model of Electroweak Interactions, arXiv:hep-ph/9412274

[25] Tevatron Electroweak Working Group, arXiv:1107.5255 [hep-ex]; http://tevewwg.fnal.gov/

[26] Y. Nambu, Phys. Rev. 117 (1960) 648.

[27] J. Goldstone, Nuov. Cim. 19 (1961) 154.

[28] J. Goldstone, A. Salam and S. Weinberg, Phys. Rev. 127 (1962) 965.

[29] F. Englert and R. Brout, Phys. Rev. Lett. 13 (1964) 321.

[30] P.W. Higgs, Phys. Rev. 145 (1966) 1156; Phys. Rev. Lett. 13 (1964) 508.

[31] G.S. Guralnik, C.R. Hagen and T.W.B. Kibble, Phys. Rev. Lett. 13 (1964) 585.

[32] T.W.B. Kibble, Phys. Rev. 155 (1967) 1554.

[33] G. 't Hooft, Nucl. Phys. B33 (1971) 173.

[34] The ALEPH, CDF, D0, DELPHI, L3, OPAL, SLD Collaborations, the LEP Electroweak Working Group, the Tevatron Electroweak Working Group and the SLD Electroweak and Heavy Flavour Groups, arXiv:1012.2367] [hep-ex]; http://www.cern.ch/LEPEWWG/.

[35] The ALEPH, DELPHI, L3, OPAL and SLD Collaborations, the LEP Electroweak Working Group and the SLD Electroweak and Heavy Flavour Groups, Phys. Rept. 427 (2006) 257.

[36] MuLan Collaboration, Phys. Rev. Lett 106 (2011) 041803.

[37] W.J. Marciano and A. Sirlin, Phys. Rev. Lett. 61 (1988) 1815.

[38] T. van Ritbergen and R.G. Stuart, Phys. Rev. Lett. 82 (1999) 488; Nucl. Phys. $B 564$ (2000) 343.

[39] A. Pak and A. Czarnecki, Phys. Rev. Lett. 100 (2008) 241807.

[40] ALEPH, DELPHI, L3 and OPAL Collaborations and the LEP Working Group for Higgs Boson Searches, Phys. Lett. $\mathbf{B 5 6 5}$ (2003) 61.

[41] A. Pich, Flavour Physics and CP violation, arXiv:1112.4094 [hep-ph].

[42] Babar Collaboration, Phys. Rev. Lett. 105 (2010) 051602.

[43] NA62 Collaboration, Phys. Lett. B698 (2011) 105.

[44] M. Antonelli et al., Eur. Phys. J. C69 (2010) 399.

[45] S. Bethke, Eur. Phys. J. C64 (2009) 689.

[46] S. Bethke et al., Workshop on Precision Measurements of $\alpha_{s}$, arXiv:1110.0016 [hep-ph].

[47] D.J. Gross and F. Wilczek, Phys. Rev. Lett. 30 (1973) 1343.

[48] H.D. Politzer, Phys. Rev. Lett. 30 (1973) 1346.

[49] T. Appelquist and J. Carazzone, Phys. Rev. D11 (1975) 2856.

[50] M. Veltman, Nucl. Phys. B123 (1977) 89.

[51] J. Bernabéu, A. Pich and A. Santamaría, Phys. Lett. B200 (1988) 569; Nucl. Phys. B363 (1991) 326.

[52] A.A. Akhundov, D. Yu. Bardin and T. Riemann, Nucl. Phys. B276 (1986) 1.

[53] W. Beenakker and W. Hollik, Z. Phys. C40 (1988) 141.

[54] B.W. Lynn and R.G. Stuart, Phys. Lett. B252 (1990) 676.

[55] The Gfitter Group, arXiv:1107.0975; http://gfitter.desy.de/Standard_Model/

[56] LHC Higgs Cross Section Working Group, https://twiki.cern.ch/twiki/bin/view/LHCPhysics/CrossSections

[57] OPAL Collaboration, Eur. Phys. J. C27 (2003) 311.

[58] CDF and D0 Collaborations, arXiv:1107.5518 [hep-ex].

[59] ATLAS Collaboration, ATLAS-CONF-2011-163 (2011).

[60] CMS Collaboration, CMS PAS HIG-11-032 (2011). 
[61] N. Cabibbo, Phys. Rev. Lett. 10 (1963) 531.

[62] M. Kobayashi and T. Maskawa, Prog. Theor. Phys. 42 (1973) 652.

[63] SINDRUM Collaboration, Nucl. Phys. B299 (1988) 1.

[64] MEG Collaboration, Phys. Rev. Lett. 107 (2011) 171801.

[65] BABAR Collaboration, Phys. Rev. Lett. 104 (2010) 021802; 103 (2009) 021801; 100 (2008) 071802; 99 (2007) 251803; 98 (2007) 061803; 95 (2005) 191801; Phys. Rev. D81 (2010) 111101; D79 (2009) 012004.

[66] BELLE Collaboration, Phys. Lett. B692 (2010) 4; B687 (2010) 139; B682 (2010) 355; B672 (2009) 317; B666 (2008) 16; B664 (2008) 35; B660 (2008) 154; B648 (2007) 341.

[67] A. Pich, Flavourdynamics, arXiv:hep-ph/9601202.

[68] J.C. Hardy and I.S. Towner, Phys. Rev. C79 (2009) 055502; C77 (2008) 025501; Rept. Prog. Phys. 73 (2010) 046301.

[69] W.J. Marciano and A. Sirlin, Phys. Rev. Lett. 96 (2006) 032002; 71 (1993) 3629.

[70] A. Czarnecki, W.J. Marciano and A. Sirlin, Phys. Rev. D70 (2004) 093006.

[71] D. Počanić et al., Phys. Rev. Lett. 93 (2004) 181803.

[72] M. Antonelli et al., Phys. Rept. 494 (2010) 197.

[73] Flavianet Kaon Working Group, Eur. Phys. J. C69 (2010) 399.

[74] V. Cirigliano, G. Ecker, H. Neufeld, A. Pich and J. Portolés, Rev. Mod. Phys. (2012), in press (arXiv:1107.6001 [hep-ph]).

[75] V. Cirigliano and H. Neufeld, Phys. Lett. B700 (2011) 7.

[76] E. Gámiz, M. Jamin, A. Pich, J. Prades and F. Schwab, Phys. Rev. Lett. 94 (2005) 011803; JHEP 0301 (2003) 060.

[77] A. Pich, Nucl. Phys. (Proc. Suppl.) 218 (2011) 89.

[78] V. Mateu and A. Pich, JHEP 0510 (2005) 041.

[79] N. Cabibbo, E.C. Swallow and R. Winston, Annu. Rev. Nucl. Part. Sci. 53 (2003) 39; Phys. Rev. Lett. 92 (2004) 251803.

[80] CLEO Collaboration, Phys. Rev. D80 (2009) 032005.

[81] J. Lahio, B.D. Pecjak and C. Schwanda, arXiv:1107.3934

[82] DELPHI Collaboration, Phys. Lett. B439 (1998) 209.

[83] Heavy Flavour Averaging Group, arXiv:1010.1589 [hep-ex]; http://www.slac.stanford.edu/xorg/hfag.

[84] C. Bernard et al., Phys. Rev. D79 (2009) 014506; PoS LATTICE2010 (2010) 311.

[85] M. Okamoto, PoS LAT2005 (2006) 013.

[86] CDF Collaboration, Phys. Rev. Lett. 95 (2005) 102002.

[87] D0 Collaboration, Phys. Rev. Lett. 100 (2008) 192003.

[88] The Tevatron Electroweak Working Group, arXiv:0908.2171 [hep-ex].

[89] N. Isgur and M. Wise, Phys. Lett. B232 (1989) 113; B237 (1990) 527.

[90] B. Grinstein, Nucl. Phys. B339 (1990) 253.

[91] E. Eichten and B. Hill, Phys. Lett. B234 (1990) 511.

[92] H. Georgi, Phys. Lett. B240 (1990) 447.

[93] M. Neubert, Phys. Lett. B264 (1991) 455.

[94] M. Luke, Phys. Lett. B252 (1990) 447.

[95] L. Wolfenstein, Phys. Rev. Lett. 51 (1983) 1945.

[96] A.J. Buras, M.E. Lautenbacher and G. Ostermaier, Phys. Rev. D50 (1994) 3433.

[97] C. Jarlskog, Phys. Rev. Lett. 55 (1985) 1039; Z. Phys. C29 (1985) 491. 
[98] CDF Collaboration, Phys. Rev. Lett. 97 (2006) 242003.

[99] LHCb Collaboration, arXiv:1112:4311 [hep-ex].

[100] CKMfitter Group, Eur. Phys. J. C41 (2005) 1; http://ckmfitter.in2p3.fr/.

[101] A.B. Carter and A.I. Sanda, Phys. Rev. Lett. 45 (1980) 952; Phys. Rev. D23 (1981) 1567.

[102] I.I. Bigi and A.I. Sanda, Nucl. Phys. B193 (1981) 85.

[103] UTfit Collaboration, JHEP 0610 (2006) 081; http://utfit.roma1.infn.it/

[104] A.J. Buras, F. Schwab and S. Uhli, Rev. Mod. Phys. 80 (2008) 965.

[105] SNO Collaboration, Phys. Rev. Lett. 89 (2002) 011301, 011302; 92 (2004) 181301; 101 (2008) 111301; Phys. Rev. C72 (2005) 055502; C75 (2007) 045502; C81 (2010) 055504; arXiv:1109.0763 [nucl-ex].

[106] R. Davis et al., Phys. Rev. Lett. 20 (1968) 1205.

[107] J.N. Bahcall, A.M. Serenelli and S. Basu, Astrophys. J. 621 (2005) L85.

[108] Super-Kamiokande Collaboration, Phys. Rev. D73 (2006) 112001; D78 (2008) 032002; D83 (2011) 052010.

[109] KamLAND Collaboration, Phys. Rev. Lett. 90 (2003) 021802; 92 (2004) 071301; 94 (2005) 081801; 100 (2008) 221803; Phys. Rev. D83 (2011) 052002.

[110] Borexino Collaboration, Phys. Lett. B658 (2008) 101; Phys. Rev. Lett. 101 (2008) 091302.

[111] Super-Kamiokande Collaboration, Phys. Rev. Lett. 81 (1998) 1562; 82 (1999) 2644, 5194; 85 (2000) 3999; 93 (2004) 101801; 97 (2006) 171801; Phys. Rev. D71 (2005) 112005; 74 (2006) 032002; D81 (2010) 092004.

[112] K2K Collaboration, Phys. Rev. D74 (2006) 072003; Phys. Rev. Lett. 90 (2003) 041801; 94 (2005) 081802.

[113] MINOS Collaboration, Phys. Rev. Lett. 97 (2006) 191801; 101 (2008) 131802; 106 (2011) 181801; 107 (2011) 021801, 181802; Phys. Rev. D84 (2011) 071103.

[114] OPERA Collaboration, Phys. Lett. B691 (2010) 138.

[115] CHOOZ Collaboration, Phys. Lett. B466 (1999) 415; Eur. Phys. J. C27 (2003) 331.

[116] T2K Collaboration, Phys. Rev. Lett. 107 (2011) 041801.

[117] DOUBLE CHOOZ Collaboration, arXiv:1112.6353 [hep-ex].

[118] T. Schwetz, M. Tortola and J.W.F. Valle, New J. Phys. 13 (2011) 063004, 109401.

[119] S. Weinberg, Phys. Rev. Lett. 43 (1979) 1566.

[120] M. Gell-Mann, P. Ramond and R. Slansky, Complex Spinors and Unified Theories, in Supergravity, eds. P. van Nieuwenhuizen and D.Z. Freedman (North Holland, Stony Brook 1979) p. 315.

[121] T. Yanagida, in Proc. Workshop on Unified Theory and Baryon Number in the Universe, eds. O. Sawada and A. Sugamoto (KEK, 1979) p. 95.

[122] D. Glenzinski (Mu2e Collaboration), AIP Conf.Proc. 1222 (2010) 383.

[123] Y. Kuno (COMET Collaboration), PoS ICHEP2010 (2010) 526.

[124] R. Barlow (PRISM/PRIME Collaboration), Nucl. Phys. B (Proc. Suppl.) 218 (2011) 44.

[125] R.D. Bolton et al., Phys. Rev. D38 (1988) 2077.

[126] J.D. Bjorken and S.D. Drell, Relativistic Quantum Mechanics, McGraw-Hill (New York, 1964). 